\title{
SOME REMARKS ON SYSTEMS OF ELLIPTIC EQUATIONS DOUBLY CRITICAL IN THE WHOLE $\mathbb{R}^{N}$
}

\author{
BOUMEDIENE ABDELLAOUI,VERONICA FELLI AND IRENEO PERAL
}

Abstract. We study the existence of different types of positive solutions to problem

$$
\left\{\begin{array}{ll}
-\Delta u-\lambda_{1} \frac{u}{|x|^{2}}-|u|^{2^{*}-2} u=\nu h(x) \alpha|u|^{\alpha-2}|v|^{\beta} u, & \text { in } \mathbb{R}^{N}, \\
-\Delta v-\lambda_{2} \frac{v}{|x|^{2}}-|v|^{2^{*}-2} v=\nu h(x) \beta|u|^{\alpha}|v|^{\beta-2} v, & \text { in } \mathbb{R}^{N},
\end{array} \quad x \in \mathbb{R}^{N}, \quad N \geq 3,\right.
$$

where $\lambda_{1}, \lambda_{2} \in\left(0, \Lambda_{N}\right), \Lambda_{N}:=\frac{(N-2)^{2}}{4}$, and $2^{*}=\frac{2 N}{N-2}$ is the critical Sobolev exponent.

A careful analysis of the behavior of Palais-Smale sequences is performed to recover compactness for some ranges of energy levels and to prove the existence of ground state solutions and mountain pass critical points of the associated functional on the Nehari manifold. A variational perturbative method is also used to study the existence of a non trivial manifold of positive solutions which bifurcates from the manifold of solutions to the uncoupled system corresponding to the unperturbed problem obtained for $\nu=0$.

\section{Introduction}

In this work we deal with the following class of systems of nonlinear elliptic equations in $\mathbb{R}^{N}$, $N \geq 3$, involving critical power nonlinearities as well as Hardy-type singular potentials, and coupled by a nonlinear term

$$
\begin{cases}-\Delta u-\lambda_{1} \frac{u}{|x|^{2}}-|u|^{2^{*}-2} u=\nu h(x) \alpha|u|^{\alpha-2}|v|^{\beta} u, & \text { in } \mathbb{R}^{N} \\ -\Delta v-\lambda_{2} \frac{v}{|x|^{2}}-|v|^{2^{*}-2} v=\nu h(x) \beta|u|^{\alpha}|v|^{\beta-2} v, & \text { in } \mathbb{R}^{N}\end{cases}
$$

where $\lambda_{1}, \lambda_{2} \in\left(0, \Lambda_{N}\right), \Lambda_{N}:=\frac{(N-2)^{2}}{4}, 2^{*}=\frac{2 N}{N-2}$ is the critical Sobolev exponent, $\nu$ is a positive parameter, and $\alpha$ and $\beta$ are positive constants such that $\alpha, \beta>1$. The mathematical interest in the Schrödinger equations appearing in the above system relies in their double criticality, due to the fact that both the exponent of the nonlinearities (which is critical in the sense of the Sobolev embeddings) and the singularities share the same order of homogeneity as the laplacian. In particular, inverse square potentials, which arise in several physical contexts (e.g. in nonrelativistic quantum mechanics, molecular physics, quantum cosmology, linearization of combustion models), do not belong to the Kato's class, hence they cannot be regarded as a lower order perturbation term. Moreover the double criticality in the potentials and in the nonlinearities produces a lack of

2000 Mathematics Subject Classification. 35D10, 35J45, 35J50, 35J60, 46E30, 46E35.

Key words and phrases. Systems elliptic equations, Compactness principles, Critical Sobolev exponent, Hardy potential, Doubly critical problems, Variational methods, Perturbation methods.

First and third authors supported by projects MTM2007-65018, MEC and CCG06-UAM/ESP-0340, Spain. Second author supported by Italy MIUR, national project Variational Methods and Nonlinear Differential Equations. 
compactness which creates some difficulty in the variational approach to the problem, i.e. in the search for solutions to system (1) as critical points of the associated functional

$$
\begin{aligned}
J_{\nu}(u, v)= & \frac{1}{2} \int_{\mathbb{R}^{N}}\left(|\nabla u(x)|^{2}+|\nabla v(x)|^{2}\right) d x-\frac{\lambda_{1}}{2} \int_{\mathbb{R}^{N}} \frac{u^{2}(x)}{|x|^{2}} d x-\frac{\lambda_{2}}{2} \int_{\mathbb{R}^{N}} \frac{v^{2}(x)}{|x|^{2}} d x \\
& -\frac{1}{2^{*}} \int_{\mathbb{R}^{N}}\left(|u(x)|^{2^{*}}+|v(x)|^{2^{*}}\right) d x-\nu \int_{\mathbb{R}^{N}} h(x)|u(x)|^{\alpha}|v(x)|^{\beta} d x
\end{aligned}
$$

defined in the product energy space $\mathcal{D}^{1,2}\left(\mathbb{R}^{N}\right) \times \mathcal{D}^{1,2}\left(\mathbb{R}^{N}\right)$, denoting by $\mathcal{D}^{1,2}\left(\mathbb{R}^{N}\right)$ the completion of $C_{0}^{\infty}\left(\mathbb{R}^{N}\right)$ with respect to the norm

$$
\|u\|_{\mathcal{D}^{1,2}\left(\mathbb{R}^{N}\right)}:=\left(\int_{\mathbb{R}^{N}}|\nabla u(x)|^{2} d x\right)^{1 / 2} .
$$

The case of a single equation has been deeply investigated in the literature. In particular, a complete classification of positive $\mathcal{D}^{1,2}\left(\mathbb{R}^{N}\right)$-solutions to

$$
-\Delta u-\lambda \frac{u}{|x|^{2}}-|u|^{2^{*}-2} u=0, \quad \text { in } \mathbb{R}^{N},
$$

is given in [28], see (4-5). We also mention that existence and multiplicity of positive solutions for related perturbative equations with a Hardy-type potential and a critical nonlinearity are obtained by variational methods in $[1,12,13,15,26]$.

The interest in systems of nonlinear Schrödinger equations is motivated by applications to nonlinear optics. More precisely, coupled nonlinear Schrödinger systems arise in the description of several physical phenomena such as the propagation of pulses in birefringent optical fibers and Kerr-like photorefractive media, see $[2,16,21,22]$. We mention that some interesting results for linearly coupled systems in the form

$$
\begin{cases}-\Delta u+u-u^{3}=\nu v, & \text { in } \mathbb{R}^{N}, \\ -\Delta v+v-v^{3}=\nu u, & \text { in } \mathbb{R}^{N},\end{cases}
$$

have been recently obtained in [7], where a variational perturbation method is used to prove the existence of multi-bump solutions, while for the case of nonlinearly coupled systems we refer to $[6,19,20,25]$ and the references therein. We also mention that concentration phenomena for singularly perturbed coupled nonlinear Schrödinger systems with potentials are studied in [23, 24].

Concerning nonlinear systems with singular potentials, we mention that in [11] existence results are obtained for the hamiltonian system

$$
\left\{\begin{array}{l}
-\Delta u=\frac{v^{p}}{|x|^{\alpha}}, \quad \text { in } \Omega, \\
-\Delta v=\frac{u^{q}}{|x|^{\beta}}, \quad \text { in } \Omega,
\end{array}\right.
$$

in a bounded domain $\Omega \subset \mathbb{R}^{N}$, under some condition on the parameters $p, q, \alpha, \beta$. Some estimates on the singularity of the solutions near 0 were obtained previously in [10] for some hamiltonian system with absorptions terms.

By variational arguments, in this work we mean to investigate how the presence of a singular potential influences the existence of nontrivial solutions to the nonlinearly coupled system (1), i.e. of solutions of the type $(\phi, \psi)$ with $\phi \not \equiv 0$ and $\psi \not \equiv 0$. In the case where $\alpha+\beta>2^{*}$, a classical Pohozaev type argument shows that if $h$ is a differentiable function such that $\langle\nabla h(x), x\rangle \geq 0$, system (1) has no finite energy solutions for $\nu>0$. Hence in the whole of this paper we assume 
that $\alpha+\beta \leq 2^{*}$. Using the Concentration Compactness Principle by P.L.Lions [17, 18] and the Mountain Pass Theorem by Ambrosetti and Rabinowitz [5], we obtain both ground state solutions to (1) (see Definition 2.1) by minimizing the functional $J_{\nu}$ on the associated Nehari manifold and mountain pass solutions. The use of such variational tools requires some compactness condition, the validity of which is a nontrivial issue for system (1) due to the presence of critical nonlinearities and inverse square potentials. To overcome such a difficulty, we will perform a careful analysis of the behavior of Palais-Smale sequences to study the possible reason of lack of compactness and to pick out the ranges of energy levels where the Palais-Smale condition holds and compactness can be recovered. We will also use a perturbative approach to study the existence of a non trivial manifold of positive solutions which bifurcates from the manifold of solutions to the uncoupled system corresponding to the unperturbed problem obtained for $\nu=0$.

The paper is organized as follow. In section 2 we analyze the behavior of the energy functional restricted to the Nehari manifold $\mathcal{N}_{\nu}$ associated to problem (1) and classify the nature of semitrivial solutions (we call semi-trivial solution any solution of the form $(\phi, 0)$ or $(0, \psi)$ ), establishing for which range of the parameters $\alpha$ and $\beta$ they are minimum or saddle points for $\left.J_{\nu}\right|_{\mathcal{N}_{\nu}}$, see Theorem 2.2.

In section 3 we deal with the subcritical case, namely we assume that $\alpha+\beta<2^{*}$ and $h$ satisfies some proper integrability condition. The analysis of the behavior of Palais-Smale sequences performed in subsection 3.1 provides enough compactness to prove Theorem 3.7, which ensures the existence of a ground state solution to (1) provided either $\nu$ is large or $\alpha, \beta \in(1,2)$. It is worth noticing that in the case where $\alpha, \beta \geq 2$, the largeness condition on $\nu$ required in Theorem 3.7 is almost optimal because, if $\nu$ is small, then the minimum of $J_{\nu}$ on the Nehari can be achieved only by semi-trivial pairs, as proved in Theorem 3.4. For $\alpha, \beta \geq 2$ and $\nu$ sufficiently small, the functional $J_{\nu}$ restricted to the Nehari manifold exhibits a mountain pass geometry, hence, thanks to the improvement of the Palais-Smale condition obtained in Lemma 3.5, we deduce in subsection 3.3 the existence of a mountain pass critical point of $J_{\nu}$ on the Nehari manifold, see Theorem 3.8.

If $\alpha<2<\beta$ and $\lambda_{1}=\lambda_{2}$, the energy functional still exhibits a mountain pass geometry and a concentration-compactness argument provides a local Palais-Smale condition. However, the existence of a suitable path in the corresponding Nehari manifold, with energy strictly below the Palais-Smale level, seems to be an open problem, see remark 3.10.

The case $\alpha+\beta=2^{*}$ treated in section 4 presents an additional difficulty due to a critical growth also in the coupling term and the consequent further lack of compactness. The coefficient $h$ of the coupling term will be assumed to be a bounded function vanishing at 0 and $\infty$, in order to exclude concentration phenomena at 0 and at $\infty$. If $h$ is radial, an improved local Palais-Smale condition implies the existence of a ground state solution if either $\alpha, \beta<2$ or $\nu$ is sufficiently large, as stated in Theorem 4.2. If $h$ is a non-radial function vanishing at 0 and $\infty$, a local Palais-Smale condition turns out to hold for $\nu$ sufficiently small, thus yielding the existence of a ground state in the case where $\alpha, \beta<2$, see Theorem 4.5. In remark 4.6 at the end of the section, we observe how a direct calculation yields solutions to (1) of the form $(\phi, c \phi)$, for $c>0$ and $\phi$ solving a suitable problem.

In the final section, we treat problem (1) in the case $\alpha+\beta \leq 2^{*}$ using the variational perturbative method developed $[3,4]$, which allows to find critical points of a perturbed functional by reducing the problem to a finite dimensional one. Under the assumption that $h$ is a bounded function with a fixed sign, compact support, and some behavior at 0 , we describe the asymptotics of any solution to (1) at the singularity and prove that (1) admits a solution for $|\nu|$ sufficiently small, see Theorem 5.6. 
Acknowledgment. Part of this work was carried out while the second author was visiting Universidad Autónoma of Madrid; she wishes to express her gratitude to Departamento de Matemáticas of Universidad Autónoma for its warm hospitality.

\section{Preliminaries}

We assume that $h$ satisfies the hypothesis

$$
h(x) \geq 0, \quad h \not \equiv 0, \quad h \in L^{1}\left(\mathbb{R}^{N}\right) \cap L^{\infty}\left(\mathbb{R}^{N}\right),
$$

and

$$
\alpha>1, \quad \beta>1, \quad \alpha+\beta \leq 2^{*} .
$$

The natural functional space to frame the analysis of (1) by variational techniques is the space $\mathcal{D}^{1,2}\left(\mathbb{R}^{N}\right)$ defined in the introduction. For $\nu=0$, system (1) is reduced to two uncoupled equations

$$
-\Delta u-\lambda_{i} \frac{u}{|x|^{2}}-|u|^{2^{*}-2} u=0, \quad i=1,2,
$$

the positive $\mathcal{D}^{1,2}\left(\mathbb{R}^{N}\right)$-solutions of which are completely classified in [28]. More precisely, in [28], it is proved that if $\lambda \in\left(0,(N-2)^{2} / 4\right)$ then problem

$$
\left\{\begin{array}{l}
-\Delta u-\frac{\lambda}{|x|^{2}} u=u^{2^{*}-1}, \quad x \in \mathbb{R}^{N}, \\
u>0 \text { in } \mathbb{R}^{N} \backslash\{0\}, \text { and } u \in \mathcal{D}^{1,2}\left(\mathbb{R}^{N}\right),
\end{array}\right.
$$

has exactly an one-dimensional $\mathcal{C}^{2}$ manifold of positive solutions given by

$$
Z_{\lambda}=\left\{z_{\mu}^{\lambda}(x)=\mu^{-\frac{N-2}{2}} z_{1}^{\lambda}\left(\frac{x}{\mu}\right), \mu>0\right\}
$$

where we denote

$$
z_{1}^{\lambda}(x)=\frac{A(N, \lambda)}{|x|^{a_{\lambda}}\left(1+|x|^{2-\frac{4 a_{\lambda}}{N-2}}\right)^{\frac{N-2}{2}}}
$$

$a_{\lambda}=\frac{N-2}{2}-\sqrt{\left(\frac{N-2}{2}\right)^{2}-\lambda}$, and $A(N, \lambda)=\left[\frac{N\left(N-2-2 a_{\lambda}\right)^{2}}{N-2}\right]$. Moreover, all solutions to (3) minimize the associated Rayleigh quotient and the minimum can be computed as:

$$
S(\lambda):=\inf _{u \in \mathcal{D}^{1,2}\left(\mathbb{R}^{N}\right) \backslash\{0\}} \frac{Q_{\lambda}(u)}{\left(\int_{\mathbb{R}^{N}}|u|^{2^{*}} d x\right)^{2 / 2^{*}}}=\frac{Q_{\lambda}\left(z_{\mu}^{\lambda}\right)}{\left(\int_{\mathbb{R}^{N}}\left|z_{\mu}^{\lambda}\right|^{2^{*}} d x\right)^{2 / 2^{*}}}=\left(1-\frac{4 \lambda}{(N-2)^{2}}\right)^{\frac{N-1}{N}} S,
$$

where $S$ is the best constant in the Sobolev inequality and $Q_{\lambda}(u)$ denotes the quadratic form

$$
Q_{\lambda}(u)=\int_{\mathbb{R}^{N}}|\nabla u|^{2} d x-\lambda \int_{\mathbb{R}^{N}} \frac{u^{2}}{|x|^{2}} d x, \quad u \in \mathcal{D}^{1,2}\left(\mathbb{R}^{N}\right),
$$

see [28]. Hence, for all $\mu>0$, the pairs $\left(z_{\mu}^{\lambda_{1}}, 0\right)$ and $\left(0, z_{\mu}^{\lambda_{2}}\right)$ solve system (1). In the sequel we will refer to this kind of solutions (i.e. solutions with one trivial component) as semi-trivial solutions. The goal of this work is to provide solutions which are neither trivial nor semi-trivial, namely solutions with both components nontrivial. Since $\lambda_{i}<\Lambda_{N}$, from Hardy's inequality it follows that $\left(Q_{\lambda_{i}}(\cdot)\right)^{1 / 2}$ is an equivalent norm to $\|\cdot\|_{\mathcal{D}^{1,2}\left(\mathbb{R}^{N}\right)}$, which will be denoted as

$$
\|w\|_{\lambda_{i}}^{2}:=Q_{\lambda_{i}}(w)=\int_{\mathbb{R}^{N}}|\nabla w|^{2}-\lambda_{i} \int_{\mathbb{R}^{N}} \frac{w^{2}}{|x|^{2}} d x
$$


while the associated scalar product will be denoted as

$$
(u, w)_{\lambda_{i}}:=\int_{\mathbb{R}^{N}} \nabla u \cdot \nabla w-\lambda_{i} \int_{\mathbb{R}^{N}} \frac{u w}{|x|^{2}} d x .
$$

Let us introduce the product space $\mathbb{D}:=\mathcal{D}^{1,2}\left(\mathbb{R}^{N}\right) \times \mathcal{D}^{1,2}\left(\mathbb{R}^{N}\right)$, endowed with the norm

$$
\|(u, v)\|_{\mathbb{D}}^{2}=\|u\|_{\lambda_{1}}^{2}+\|v\|_{\lambda_{2}}^{2} .
$$

As set in the introduction, solutions to (1) can be found as critical points of the functional

$$
J_{\nu}: \mathbb{D} \rightarrow \mathbb{R}, \quad J_{\nu}(u, v)=I_{\lambda_{1}}(u)+I_{\lambda_{2}}(v)-\nu \int_{\mathbb{R}^{N}} h(x)|u|^{\alpha}|v|^{\beta} d x,
$$

where

$$
I_{\lambda_{i}}(w)=\frac{1}{2} Q_{\lambda_{i}}(w)-\frac{1}{2^{*}} \int_{\mathbb{R}^{N}}|w|^{2^{*}} d x, \quad i=1,2 .
$$

We observe that $J_{\nu} \in C^{1}(\mathbb{D}, \mathbb{R})$. Let $\mathcal{N}_{\nu}$ be the Nehari manifold associated to $J_{\nu}$, namely $\mathcal{N}_{\nu}$ is defined by

$$
\mathcal{N}_{\nu}=\left\{(u, v) \in \mathbb{D} \backslash\{(0,0)\}:\left\langle J_{\nu}^{\prime}(u, v),(u, v)\right\rangle=0\right\},
$$

where $J_{\nu}^{\prime}(u, v)$ denotes the Fréchet derivative of $J_{\nu}$ at $(u, v)$, and $\langle\cdot, \cdot\rangle$ is the duality product between $\mathbb{D}$ and its dual space $\mathbb{D}^{\star}$. A direct computation shows that for all $(u, v) \in \mathbb{D} \backslash\{(0,0)\}$ there exists $t_{[\nu,(u, v)]}>0$ such that $t_{[\nu,(u, v)]}(u, v) \in \mathcal{N}_{\nu}$. More precisely $t_{[\nu,(u, v)]}$ is the unique positive solution to the following algebraic equation in $t$ :

$$
\|(u, v)\|_{\mathbb{D}}^{2}=t^{2^{*}-2}\left(\int_{\mathbb{R}^{N}}|u|^{2^{*}} d x+\int_{\mathbb{R}^{N}}|v|^{2^{*}} d x\right)+\nu(\alpha+\beta) t^{\alpha+\beta-2} \int_{\mathbb{R}^{N}} h(x)|u|^{\alpha}|v|^{\beta} d x .
$$

Then, for $(u, v) \in \mathbb{D} \backslash\{(0,0)\}$,

$$
\begin{aligned}
J_{\nu}\left(t_{[\nu,(u, v)]}(u, v)\right)= & \frac{1}{N} t_{[\nu,(u, v)]}^{2^{*}}\left(\int_{\mathbb{R}^{N}}|u|^{2^{*}} d x+\int_{\mathbb{R}^{N}}|v|^{2^{*}} d x\right) \\
& +\nu \frac{\alpha+\beta-2}{2} t_{[\nu,(u, v)]}^{\alpha+\beta} \int_{\mathbb{R}^{N}} h(x)|u|^{\alpha}|v|^{\beta} d x>0 .
\end{aligned}
$$

Notice that, by the homogeneity of $J_{\nu}$, there exists a positive constant $r_{\nu}$ such that

$$
\|(u, v)\|_{\mathbb{D}} \geq r_{\nu} \quad \text { for all }(u, v) \in \mathcal{N}_{\nu}
$$

and

$$
\begin{aligned}
J_{\nu}(u, v) & =\left(\frac{1}{2}-\frac{1}{\alpha+\beta}\right)\|(u, v)\|_{\mathbb{D}}^{2}+\left(\frac{1}{\alpha+\beta}-\frac{1}{2^{*}}\right)\left(\int_{\mathbb{R}^{N}}|u|^{2^{*}} d x+\int_{\mathbb{R}^{N}}|v|^{2^{*}} d x\right) \\
& \geq\left(\frac{1}{2}-\frac{1}{\alpha+\beta}\right) r_{\nu}^{2} \quad \text { for all }(u, v) \in \mathcal{N}_{\nu},
\end{aligned}
$$

i.e. $\left.\quad J_{\nu}\right|_{\mathcal{N}_{\nu}}$ is bounded from below away from zero. By ground state solutions to (1) we mean solutions with minimal energy in the Nehari manifold, as we define below.

Definition 2.1. A ground state solution to (1) is a critical point $\left(u_{0}, v_{0}\right)$ of $J_{\nu}$ in $\mathbb{D}$ which solves the following minimization problem

$$
c^{*}(\nu):=\inf _{(u, v) \in \mathcal{N}_{\nu}} J_{\nu}(u, v)=J_{\nu}\left(u_{0}, v_{0}\right) .
$$


The following theorem clarifies how the nature of semi-trivial solutions depends on the dimension and the values of the parameters. This kind of analysis will permit us to recognize how the geometry of the energy functional looks like depending on the values of the parameters $\alpha$ and $\beta$. We notice that a direct calculation yields the energy levels of semi-trivial solutions which turn out to be

$$
J_{\nu}\left(z_{\mu}^{\lambda_{1}}, 0\right)=\frac{1}{N} S\left(\lambda_{1}\right)^{N / 2}, \quad J_{\nu}\left(0, z_{\mu}^{\lambda_{2}}\right)=\frac{1}{N} S\left(\lambda_{2}\right)^{N / 2}, \quad \text { for all } \mu>0 .
$$

Theorem 2.2. Let us assume that $(\mathbf{H})$ and (2) are satisfied.

i) If

$$
\text { either } \beta>2 \text { or }\left\{\begin{array}{l}
\beta=2, \\
\nu \text { is sufficiently small, }
\end{array}\right.
$$

then, for all $\mu>0,\left(z_{\mu}^{\lambda_{1}}, 0\right)$ is a local minimum point of $J_{\nu}$ in $\mathcal{N}_{\nu}$.

ii) If

$$
\text { either } \alpha>2 \text { or }\left\{\begin{array}{l}
\alpha=2, \\
\nu \text { is sufficiently small, }
\end{array}\right.
$$

then, for all $\mu>0,\left(0, z_{\mu}^{\lambda_{2}}\right)$ is a local minimum point of $J_{\nu}$ in $\mathcal{N}_{\nu}$.

iii) If

$$
\text { either } \beta<2 \text { or }\left\{\begin{array}{l}
\beta=2, \\
\nu \text { is sufficiently large, }
\end{array}\right.
$$

then, for all $\mu>0,\left(z_{\mu}^{\lambda_{1}}, 0\right)$ is a saddle point for $J_{\nu}$ in $\mathcal{N}_{\nu}$.

iv) If

$$
\text { either } \alpha<2 \text { or }\left\{\begin{array}{l}
\alpha=2, \\
\nu \text { is sufficiently large, }
\end{array}\right.
$$

then, for all $\mu>0,\left(0, z_{\mu}^{\lambda_{2}}\right)$ is a saddle point for $J_{\nu}$ in $\mathcal{N}_{\nu}$.

Proof. To prove i), assume that $\mu>0, \beta>2$ and $\left(z_{\mu}^{\lambda_{1}}+\phi, \psi\right) \in \mathcal{N}_{\nu}$, i.e.

(12) $\left\|z_{\mu}^{\lambda_{1}}+\phi\right\|_{\lambda_{1}}^{2}+\|\psi\|_{\lambda_{2}}^{2}=\int_{\mathbb{R}^{N}}\left|z_{\mu}^{\lambda_{1}}+\phi\right|^{2^{*}} d x+\int_{\mathbb{R}^{N}}|\psi|^{2^{*}} d x+\nu(\alpha+\beta) \int_{\mathbb{R}^{N}} h(x)\left|z_{\mu}^{\lambda_{1}}+\phi\right|^{\alpha}|\psi|^{\beta} d x$.

Let $t=t(\phi, \psi)>0$ be such that $t\left(z_{\mu}^{\lambda_{1}}+\phi\right) \in N_{\lambda_{1}}$, where $N_{\lambda_{1}}$ denotes the Nehari manifold associated to $I_{\lambda_{1}}$, namely $N_{\lambda_{1}}$ is defined by

$$
N_{\lambda_{1}}=\left\{u \in \mathcal{D}^{1,2}\left(\mathbb{R}^{N}\right) \backslash\{0\}:\|u\|_{\lambda_{1}}^{2}=\|u\|_{L^{2^{*}}}^{2^{*}}\right\} .
$$

A direct calculation yields

$$
t=t(\phi, \psi)=\left[\frac{\left\|z_{\mu}^{\lambda_{1}}+\phi\right\|_{\lambda_{1}}^{2}}{\left\|z_{\mu}^{\lambda_{1}}+\phi\right\|_{L^{2^{*}}}^{2^{*}}}\right]^{\frac{1}{2^{*}-2}}=\left[1-\frac{\|\psi\|_{\lambda_{2}}^{2}-\|\psi\|_{L^{2^{*}}}^{2^{*}}-\nu(\alpha+\beta) \int_{\mathbb{R}^{N}} h(x)\left|z_{\mu}^{\lambda_{1}}+\phi\right|^{\alpha}|\psi|^{\beta} d x}{\left\|z_{\mu}^{\lambda_{1}}+\phi\right\|_{L^{2^{*}}}^{2^{*}}}\right]^{\frac{1}{2^{*}-2}}
$$

In particular, since $\beta>2$, we have that

$$
\begin{aligned}
& t^{2}(\phi, \psi)=1-\frac{2}{2^{*}-2} \frac{\|\psi\|_{\lambda_{2}}^{2}(1+o(1))}{\left\|z_{\mu}^{\lambda_{1}}+\phi\right\|_{L^{2^{*}}}^{2 *}}, \quad \text { as }\|(\phi, \psi)\|_{\mathbb{D}} \rightarrow 0, \\
& t^{2^{*}}(\phi, \psi)=1-\frac{2^{*}}{2^{*}-2} \frac{\|\psi\|_{\lambda_{2}}^{2}(1+o(1))}{\left\|z_{\mu}^{\lambda_{1}}+\phi\right\|_{L^{2^{*}}}^{2^{*}}}, \quad \text { as }\|(\phi, \psi)\|_{\mathbb{D}} \rightarrow 0 .
\end{aligned}
$$

Since $z_{\mu}^{\lambda_{1}}$ achieves the minimum of $I_{\lambda_{1}}=J_{\nu}(\cdot, 0)$ on $N_{\lambda_{1}}$, there holds

$$
J_{\nu}\left(t\left(z_{\mu}^{\lambda_{1}}+\phi\right), 0\right)-J_{\nu}\left(z_{\mu}^{\lambda_{1}}, 0\right) \geq 0 .
$$


On the other hand, from (12-15) we deduce that

$$
\begin{gathered}
J_{\nu}\left(z_{\mu}^{\lambda_{1}}+\phi, \psi\right)-J_{\nu}\left(t\left(z_{\mu}^{\lambda_{1}}+\phi\right), 0\right)=\frac{1}{2}\left(1-t^{2}(\phi, \psi)\right)\left\|z_{\mu}^{\lambda_{1}}+\phi\right\|_{\lambda_{1}}^{2} \\
-\frac{1}{2^{*}}\left(1-t^{2^{*}}(\phi, \psi)\right)\left[\left\|z_{\mu}^{\lambda_{1}}+\phi\right\|_{\lambda_{1}}^{2}+\|\psi\|_{\lambda_{2}}^{2}-\|\psi\|_{L^{2^{*}}}^{2^{*}}-\nu(\alpha+\beta) \int_{\mathbb{R}^{N}} h(x)\left|z_{\mu}^{\lambda_{1}}+\phi\right|^{\alpha}|\psi|^{\beta} d x\right] \\
+\frac{1}{2}\|\psi\|_{\lambda_{2}}^{2}-\frac{1}{2^{*}}\|\psi\|_{L^{2^{*}}\left(\mathbb{R}^{N}\right)}^{2^{*}}-\nu \int_{\mathbb{R}^{N}} h(x)\left|z_{\mu}^{\lambda_{1}}+\phi\right|^{\alpha}|\psi|^{\beta} d x=\frac{1}{2}\|\psi\|_{\lambda_{2}}^{2}(1+o(1))
\end{gathered}
$$

as $\|(\phi, \psi)\|_{\mathbb{D}} \rightarrow 0$. Hence

$$
J_{\nu}\left(z_{\mu}^{\lambda_{1}}+\phi, \psi\right)-J_{\nu}\left(t\left(z_{\mu}^{\lambda_{1}}+\phi\right), 0\right) \geq 0
$$

provided $\left(z_{\mu}^{\lambda_{1}}+\phi, \psi\right)$ is sufficiently closed to $\left(z_{\mu}^{\lambda_{1}}, 0\right)$ in $\mathbb{D}$. We notice that the inequality in $(17)$ is strict if $\psi \neq \equiv 0$. From (16) and (17), we conclude that

$$
J_{\nu}\left(z_{\mu}^{\lambda_{1}}+\phi, \psi\right)-J_{\nu}\left(z_{\mu}^{\lambda_{1}}, 0\right) \geq 0
$$

for any $\left(z_{\mu}^{\lambda_{1}}+\phi, \psi\right) \in \mathcal{N}_{\nu}$ sufficiently closed to $\left(z_{\mu}^{\lambda_{1}}, 0\right)$ (with strict inequality holding outside the manifold $\left.Z_{\lambda_{1}} \times\{0\}\right)$, i.e. $\left(z_{\mu}^{\lambda_{1}}, 0\right)$ is a local minimum point of $J_{\nu}$ in $\mathcal{N}_{\nu}$. In the case $\beta=2$, we obtain that

$$
J_{\nu}\left(z_{\mu}^{\lambda_{1}}+\phi, \psi\right)-J_{\nu}\left(t\left(z_{\mu}^{\lambda_{1}}+\phi\right), 0\right)=\left(\frac{1}{2}\|\psi\|_{\lambda_{2}}^{2}-\nu \int_{\mathbb{R}^{N}} h(x)\left|z_{\mu}^{\lambda_{1}}+\phi\right|^{\alpha}|\psi|^{2} d x\right)(1+o(1))
$$

which still is nonnegative provided $\|(\phi, \psi)\|_{\mathbb{D}}$ and $\nu$ are sufficiently small. The proof of ii) works in the same way as i), hence we omit it.

Let us now prove iii). Assume $\beta<2$. Let us fix $\nu>0, \mu>0$, and $u \in \mathcal{D}^{1,2}\left(\mathbb{R}^{N}\right) \backslash\{0\}$. For any $t \in \mathbb{R}$, let $s(t)$ be the unique positive number such that

$$
\left(s(t) z_{\mu}^{\lambda_{1}}, s(t) t u\right) \in \mathcal{N}_{\nu} .
$$

From $(7)$, the function $s: \mathbb{R} \rightarrow(0,+\infty)$ is implicitly defined by the identity

$$
\begin{aligned}
\left\|z_{\mu}^{\lambda_{1}}\right\|_{\lambda_{1}}^{2}+t^{2}\|u\|_{\lambda_{2}}^{2}= & {[s(t)]^{2^{*}-2}\left(\int_{\mathbb{R}^{N}}\left|z_{\mu}^{\lambda_{1}}\right|^{2^{*}} d x+|t|^{2^{*}} \int_{\mathbb{R}^{N}}|u|^{2^{*}} d x\right) } \\
& +\nu(\alpha+\beta)[s(t)]^{\alpha+\beta-2}|t|^{\beta} \int_{\mathbb{R}^{N}} h(x)\left|z_{\mu}^{\lambda_{1}}\right|^{\alpha}|u|^{\beta} d x, \quad t \in \mathbb{R} .
\end{aligned}
$$

We notice that $s(0)=1$. Moreover, from the Implicit Function Theorem it follows that $s \in C^{1}(\mathbb{R})$ and

$$
\begin{aligned}
& s^{\prime}(t) \\
& \quad=\frac{2 t\|u\|_{\lambda_{2}}^{2}-2^{*}[s(t)]^{2^{*}-2}|t|^{2^{*}-2} t\|u\|_{L^{2^{*}}}^{2^{*}}-\beta \nu(\alpha+\beta)[s(t)]^{\alpha+\beta-2}|t|^{\beta-2} t \int_{\mathbb{R}^{N}} h\left|z_{\mu}^{\lambda_{1}}\right|^{\alpha}|u|^{\beta}}{\left(2^{*}-2\right)[s(t)]^{2^{*}-3}\left(\left\|z_{\mu}^{\lambda_{1}}\right\|_{L^{2^{*}}}^{2^{*}}+|t|^{2^{*}}\|u\|_{L^{2^{*}}}^{2^{*}}+\nu(\alpha+\beta)(\alpha+\beta-2)[s(t)]^{\alpha+\beta-3}|t|^{\beta} \int_{\mathbb{R}^{N}} h\left|z_{\mu}^{\lambda_{1}}\right| \alpha|u|^{\beta}\right.}
\end{aligned}
$$

for all $t \in \mathbb{R}$. Hence, since $\beta<2$,

$$
s^{\prime}(t)=-\left[\frac{\beta \nu(\alpha+\beta) \int_{\mathbb{R}^{N}} h\left|z_{\mu}^{\lambda_{1}}\right|^{\alpha}|u|^{\beta}}{\left(2^{*}-2\right)\left\|z_{\mu}^{\lambda_{1}}\right\|_{L^{2^{*}}}^{2^{*}}}\right]|t|^{\beta-2} t(1+o(1)) \quad \text { as } t \rightarrow 0,
$$

and, consequently,

$$
s(t)=1-\left[\frac{\nu(\alpha+\beta) \int_{\mathbb{R}^{N}} h\left|z_{\mu}^{\lambda_{1}}\right|^{\alpha}|u|^{\beta}}{\left(2^{*}-2\right)\left\|z_{\mu}^{\lambda_{1}}\right\|_{L^{2^{*}}}^{2^{*}}}\right]|t|^{\beta}(1+o(1)) \quad \text { as } t \rightarrow 0 .
$$


In particular, there holds that

$$
[s(t)]^{2^{*}}=1-\left[\frac{N \nu(\alpha+\beta) \int_{\mathbb{R}^{N}} h\left|z_{\mu}^{\lambda_{1}}\right|^{\alpha}|u|^{\beta}}{2\left\|z_{\mu}^{\lambda_{1}}\right\|_{L^{2^{*}}}^{2^{*}}}\right]|t|^{\beta}(1+o(1)) \quad \text { as } t \rightarrow 0 .
$$

From (8) and (18), we deduce that

$$
\begin{aligned}
& J_{\nu}\left(s(t) z_{\mu}^{\lambda_{1}}, s(t) t u\right)-J_{\nu}\left(z_{\mu}^{\lambda_{1}}, 0\right) \\
& \quad=\frac{[s(t)]^{2^{*}}-1}{N}\left\|z_{\mu}^{\lambda_{1}}\right\|_{L^{2^{*}}}^{2^{*}}+\frac{[s(t)]^{2^{*}}}{N}|t|^{2^{*}}\|u\|_{L^{2^{*}}}^{2^{*}}+\nu \frac{\alpha+\beta-2}{2}|t|^{\beta}[s(t)]^{\alpha+\beta} \int_{\mathbb{R}^{N}} h\left|z_{\mu}^{\lambda_{1}}\right|^{\alpha}|u|^{\beta} \\
& \quad=-\frac{\nu(\alpha+\beta)}{2}|t|^{\beta} \int_{\mathbb{R}^{N}} h(x)\left|z_{\mu}^{\lambda_{1}}\right|^{\alpha}|u|^{\beta} d x+\nu \frac{\alpha+\beta-2}{2}|t|^{\beta} \int_{\mathbb{R}^{N}} h\left|z_{\mu}^{\lambda_{1}}\right|^{\alpha}|u|^{\beta}+o\left(|t|^{\beta}\right) \\
& \quad=-\nu|t|^{\beta} \int_{\mathbb{R}^{N}} h\left|z_{\mu}^{\lambda_{1}}\right|^{\alpha}|u|^{\beta}+o\left(|t|^{\beta}\right) \quad \text { as } t \rightarrow 0 .
\end{aligned}
$$

Hence

$$
J_{\nu}\left(s(t) z_{\mu}^{\lambda_{1}}, s(t) t u\right)-J_{\nu}\left(z_{\mu}^{\lambda_{1}}, 0\right)<0 \text { for } t \neq 0 \text { small }
$$

i.e. $\left(z_{\mu}^{\lambda_{1}}, 0\right)$ is a local strict maximum point for $J_{\nu}$ along a path living in the Nehari manifold $\mathcal{N}_{\nu}$. On the other hand, for all $w \in N_{\lambda_{1}}$ (see (13)), we have that $(w, 0) \in \mathcal{N}_{\nu}$. Furthermore, the pairs $\left\{\left(z_{\sigma}^{\lambda_{1}}, 0\right): \sigma>0\right\}$ are the only minimizers of $J_{\nu}(\cdot, 0)$ on $N_{\lambda_{1}}$. Then

$$
J_{\nu}(w, 0)-J_{\nu}\left(z_{\mu}^{\lambda_{1}}, 0\right)>0 \text { for all } w \in N_{\lambda_{1}} \backslash\left\{\left(z_{\sigma}^{\lambda_{1}}, 0\right): \sigma>0\right\},
$$

i.e. $\left(z_{\mu}^{\lambda_{1}}, 0\right)$ is a local minimum point for $J_{\nu}$ restricted to $N_{\lambda_{1}} \times\{0\} \subset \mathcal{N}_{\nu}$. From (19) and (20) we deduce that $\left(z_{\mu}^{\lambda_{1}}, 0\right)$ is a saddle point for $J_{\nu}$ in $\mathcal{N}_{\nu}$. The above argument can be adapted to the case $\beta=2$ just by requiring that $\nu$ is sufficiently large. Indeed, in this case

$$
s^{\prime}(t)=-2\left[\frac{\nu(\alpha+2) \int_{\mathbb{R}^{N}} h\left|z_{\mu}^{\lambda_{1}}\right|^{\alpha}|u|^{2}-\|u\|_{\lambda_{2}}^{2}}{\left(2^{*}-2\right)\left\|z_{\mu}^{\lambda_{1}}\right\|_{L^{2^{*}}}^{2^{*}}}\right] t(1+o(1)) \quad \text { as } t \rightarrow 0,
$$

and hence

$$
J_{\nu}\left(s(t) z_{\mu}^{\lambda_{1}}, s(t) t u\right)-J_{\nu}\left(z_{\mu}^{\lambda_{1}}, 0\right)=\left(\frac{1}{2}\|u\|_{\lambda_{2}}^{2}-\nu \int_{\mathbb{R}^{N}} h\left|z_{\mu}^{\lambda_{1}}\right|^{\alpha}|u|^{2}\right)|t|^{2}+o\left(|t|^{2}\right) \quad \text { as } t \rightarrow 0 .
$$

Therefore (19) holds provided $\nu$ is sufficiently large. The proof of iv) works in the same way as iii), hence we omit it.

Remark 2.3. We notice that, from the proof of Theorem 2.2, there results that, under the assumption of statement $i), J_{\nu}(u, v)>J_{\nu}\left(z_{\mu}^{\lambda_{1}}, 0\right)$ for all $(u, v) \in \mathcal{N}_{\nu} \backslash\left(Z_{\lambda_{1}} \times\{0\}\right)$ sufficiently closed to $\left(z_{\mu}^{\lambda_{1}}, 0\right)$, while, under the assumption of statement ii), $J_{\nu}(u, v)>J_{\nu}\left(0, z_{\mu}^{\lambda_{2}}\right)$ for all $(u, v) \in \mathcal{N}_{\nu} \backslash\left(\{0\} \times Z_{\lambda_{2}}\right)$ sufficiently closed to $\left(0, z_{\mu}^{\lambda_{2}}\right)$.

\section{Subcritical coupling term}

In this section we assume that $h$ satisfies $(\mathbf{H})$ and deal with system (1) with a subcritical coupling term, i.e. in the case

$$
\alpha>1, \quad \beta>1, \quad \alpha+\beta<2^{*} .
$$

The minimization of $J_{\nu}$ on the Nehari manifold is a non trivial issue, as the lack of compactness of the embedding $\mathcal{D}^{1,2}\left(\mathbb{R}^{N}\right) \hookrightarrow L^{2^{*}}\left(\mathbb{R}^{N}\right)$ could lead to non-convergence of Palais-Smale sequences. To overcome this difficulty, a Concentration-Compactness argument can be employed to understand 
at which levels of energy compactness can be lost and at which ones Palais-Smale condition can be recovered.

3.1. Analysis of Palais-Smale sequences. In this subsection, we perform a careful analysis of the behavior of minimizing sequences with the aid of P. L. Lions Concentration-Compactness principle [17, 18], which allows to recover compactness below some critical threshold. Let us first notice that any Palais-Smale sequence for $J_{\nu}$ constrained on the Nehari manifold is actually a Palais-Smale sequence for the unconstrained functional.

Lemma 3.1. Let us assume that $(\mathbf{H})$ and (21) are satisfied and that $\left\{\left(u_{n}, v_{n}\right)\right\}_{n \in \mathbb{N}} \subset \mathcal{N}_{\nu}$ is a Palais-Smale sequence for the constrained functional $\left.J_{\nu}\right|_{\mathcal{N}_{\nu}}$ at level $c \in \mathbb{R}$, i.e.

$$
J_{\nu}\left(u_{n}, v_{n}\right) \rightarrow c \quad \text { in } \mathbb{R} \quad \text { and } \quad\left(\left.J_{\nu}\right|_{\mathcal{N}_{\nu}}\right)^{\prime}\left(u_{n}, v_{n}\right) \rightarrow 0 \quad \text { in the dual space } \mathbb{D}^{\star} \text {. }
$$

Then $\left\{\left(u_{n}, v_{n}\right)\right\}_{n \in \mathbb{N}}$ is a bounded Palais-Smale sequence for the unconstrained functional, i.e.

$$
J_{\nu}^{\prime}\left(u_{n}, v_{n}\right) \rightarrow 0 \quad \text { in } \mathbb{D}^{\star} .
$$

Proof. From (22) and (10) it follows that

$$
\begin{aligned}
c+o(1)=J_{\nu}\left(u_{n}, v_{n}\right) & =\left(\frac{1}{2}-\frac{1}{\alpha+\beta}\right)\left\|\left(u_{n}, v_{n}\right)\right\|_{\mathbb{D}}^{2}+\left(\frac{1}{\alpha+\beta}-\frac{1}{2^{*}}\right)\left(\int_{\mathbb{R}^{N}}\left|u_{n}\right|^{2^{*}} d x+\int_{\mathbb{R}^{N}}\left|v_{n}\right|^{2^{*}} d x\right) \\
& \geq\left(\frac{1}{2}-\frac{1}{\alpha+\beta}\right)\left\|\left(u_{n}, v_{n}\right)\right\|_{\mathbb{D}}^{2},
\end{aligned}
$$

and hence $\left\{\left(u_{n}, v_{n}\right)\right\}_{n \in \mathbb{N}}$ is bounded in $\mathbb{D}$. Setting

$$
G_{\nu}(u, v)=\left\langle J_{\nu}^{\prime}(u, v),(u, v)\right\rangle,
$$

a direct calculation yields, for any $(u, v) \in \mathcal{N}_{\nu}$,

$$
\left\langle G_{\nu}^{\prime}(u, v),(u, v)\right\rangle=(2-\alpha-\beta)\|(u, v)\|_{\mathbb{D}}^{2}+\left(\alpha+\beta-2^{*}\right)\left(\int_{\mathbb{R}^{N}}|u|^{2^{*}} d x+\int_{\mathbb{R}^{N}}|v|^{2^{*}} d x\right)
$$

and then, in view of (9),

$$
\left\langle G_{\nu}^{\prime}(u, v),(u, v)\right\rangle \leq-(\alpha+\beta-2) r_{\nu}^{2} \quad \text { for all }(u, v) \in \mathcal{N}_{\nu} .
$$

Let $\left\{\mu_{n}\right\}_{n \in \mathbb{N}} \subset \mathbb{R}$ be a sequence of multipliers satisfying

$$
J_{\nu}^{\prime}\left(u_{n}, v_{n}\right)=\left(\left.J_{\nu}\right|_{\mathcal{N}_{\nu}}\right)^{\prime}\left(u_{n}, v_{n}\right)+\mu_{n} G_{\nu}^{\prime}\left(u_{n}, v_{n}\right)
$$

Testing the above identity with $\left(u_{n}, v_{n}\right)$ and using (22), (23), the definition of Nehari manifold, and boundedness of $\left\{\left(u_{n}, v_{n}\right)\right\}$, we obtain that $\lim _{n \rightarrow+\infty} \mu_{n}=0$. Being $G_{\nu}^{\prime}\left(u_{n}, v_{n}\right)$ bounded, we conclude that $J_{\nu}^{\prime}\left(u_{n}, v_{n}\right)=o(1)$ as $n \rightarrow+\infty$.

We now establish in Lemma 3.2 a Palais-Smale condition which holds below some critical threshold independently of the value of $\nu$.

Lemma 3.2. Let us assume that $(\mathbf{H})$ and (21) are satisfied and that $\left\{\left(u_{n}, v_{n}\right)\right\}_{n \in \mathbb{N}} \subset \mathbb{D}$ is a Palais-Smale sequence for $J_{\nu}$ al level $c \in \mathbb{R}$, i.e.

$$
J_{\nu}\left(u_{n}, v_{n}\right) \rightarrow c \quad \text { in } \mathbb{R} \quad \text { and } \quad J_{\nu}^{\prime}\left(u_{n}, v_{n}\right) \rightarrow 0 \quad \text { in the dual space } \mathbb{D}^{\star} .
$$

Then

i) there exists $C>0$ such that, for all $n,\left\|\left(u_{n}, v_{n}\right)\right\|_{\mathbb{D}} \leq C$;

ii) if $c<\frac{1}{N}\left(\min \left\{S\left(\lambda_{1}\right), S\left(\lambda_{2}\right)\right\}\right)^{\frac{N}{2}}$, then, up to a subsequence, $\left(u_{n}, v_{n}\right) \rightarrow(u, v)$ strongly in $\mathbb{D}$. 
Proof. From (24), we obtain that

$$
J_{\nu}\left(u_{n}, v_{n}\right)-\frac{1}{\alpha+\beta}\left\langle J_{\nu}^{\prime}\left(u_{n}, v_{n}\right),\left(u_{n}, v_{n}\right)\right\rangle=c+o(1)+o\left(\left\|\left(u_{n}, v_{n}\right)\right\|_{\mathbb{D}}\right) \quad \text { as } \quad n \rightarrow \infty .
$$

Hence

$$
\begin{aligned}
\left(\frac{1}{2}-\frac{1}{\alpha+\beta}\right)\left\|\left(u_{n}, v_{n}\right)\right\|_{\mathbb{D}}^{2} & \leq\left(\frac{1}{2}-\frac{1}{\alpha+\beta}\right)\left\|\left(u_{n}, v_{n}\right)\right\|_{\mathbb{D}}^{2}+\left(\frac{1}{\alpha+\beta}-\frac{1}{2^{*}}\right)\left(\int_{\mathbb{R}^{N}}\left|u_{n}\right|^{2^{*}} d x+\int_{\mathbb{R}^{N}}\left|v_{n}\right|^{2^{*}} d x\right) \\
& =c+o(1)+o\left(\left\|\left(u_{n}, v_{n}\right)\right\|_{\mathbb{D}}\right) \quad \text { as } n \rightarrow \infty,
\end{aligned}
$$

and therefore we conclude that $\left\{\left(u_{n}, v_{n}\right)\right\}_{n \in \mathbb{N}}$ is bounded in $\mathbb{D}$. Then, there exist $\left(u_{0}, v_{0}\right) \in \mathbb{D}$ and a subsequence, still denoted as $\left\{\left(u_{n}, v_{n}\right)\right\}_{n \in \mathbb{N}}$, such that

$$
\begin{aligned}
& \left(u_{n}, v_{n}\right) \rightarrow\left(u_{0}, v_{0}\right) \text { weakly in } \mathbb{D}, \quad\left(u_{n}, v_{n}\right) \rightarrow\left(u_{0}, v_{0}\right) \text { a.e. in } \mathbb{R}^{N}, \\
& \text { and }\left(u_{n}, v_{n}\right) \rightarrow\left(u_{0}, v_{0}\right) \text { strongly in } L_{\text {loc }}^{\alpha}\left(\mathbb{R}^{N}\right), \quad \text { for all } \alpha \in\left[1,2^{*}\right) .
\end{aligned}
$$

In view of the Concentration Compactness Principle by P. L. Lions, (see [17] and [18]), there exist a subsequence, still denoted as $\left\{\left(u_{n}, v_{n}\right)\right\}_{n \in \mathbb{N}}$, two at most countable sets $\mathcal{J}$ and $\mathcal{K}$, set of points $\left\{x_{j} \in \mathbb{R}^{N} \backslash\{0\}, j \in \mathcal{J}\right\}$ and $\left\{y_{k} \in \mathbb{R}^{N} \backslash\{0\}, k \in \mathcal{K}\right\}$, real numbers $\mu_{j}, \rho_{j}, j \in \mathcal{J}, \bar{\mu}_{k}, \bar{\rho}_{k}, k \in \mathcal{K}$, $\mu_{0}, \rho_{0}, \gamma_{0}, \bar{\mu}_{0}, \bar{\rho}_{0}$, and $\bar{\gamma}_{0}$, such that the following convergences hold in the sense of measures

$$
\left\{\begin{array}{l}
\left|\nabla u_{n}\right|^{2} \quad \rightarrow \quad d \mu \geq\left|\nabla u_{0}\right|^{2}+\sum_{j \in \mathcal{J}} \mu_{j} \delta_{x_{j}}+\mu_{0} \delta_{0}, \\
\left|\nabla v_{n}\right|^{2} \rightarrow d \bar{\mu} \geq\left|\nabla v_{0}\right|^{2}+\sum_{k \in \mathcal{K}} \bar{\mu}_{k} \delta_{y_{k}}+\bar{\mu}_{0} \delta_{0}, \\
\left|u_{n}\right|^{2^{*}} \rightarrow d \rho=\left|u_{0}\right|^{2^{*}}+\sum_{j \in \mathcal{J}} \rho_{j} \delta_{x_{j}}+\rho_{0} \delta_{0}, \\
\left|v_{n}\right|^{2^{*}} \rightarrow d \bar{\rho}=\left|v_{0}\right|^{2^{*}}+\sum_{k \in \mathcal{K}} \bar{\rho}_{k} \delta_{y_{k}}+\bar{\rho}_{0} \delta_{0}, \\
\frac{u_{n}^{2}}{|x|^{2}} \rightarrow d \gamma=\frac{u_{0}^{2}}{|x|^{2}}+\gamma_{0} \delta_{0}, \\
\frac{v_{n}^{2}}{|x|^{2}}-d \bar{\gamma}=\frac{v_{0}^{2}}{|x|^{2}}+\bar{\gamma}_{0} \delta_{0} .
\end{array}\right.
$$

From above and Sobolev's and Hardy's inequalities it follows easily that

$$
\begin{array}{lll}
S \rho_{j}^{\frac{2}{2 *}} \leq \mu_{j} & \text { for all } j \in \mathcal{J} \cup\{0\}, & \Lambda_{N} \gamma_{0} \leq \mu_{0}, \\
S \bar{\rho}_{k}^{\frac{2}{2 *}} \leq \bar{\mu}_{k} & \text { for all } k \in \mathcal{K} \cup\{0\}, & \Lambda_{N} \bar{\gamma}_{0} \leq \bar{\mu}_{0} .
\end{array}
$$

Concentration at infinity of the sequence $\left\{u_{n}\right\}_{n \in \mathbb{N}}$ is described by the following quantities:

$$
\rho_{\infty}:=\lim _{R \rightarrow \infty} \limsup _{n \rightarrow \infty} \int_{|x|>R}\left|u_{n}\right|^{2^{*}} d x, \quad \mu_{\infty}=\lim _{R \rightarrow \infty} \limsup _{n \rightarrow \infty} \int_{|x|>R}\left|\nabla u_{n}\right|^{2} d x,
$$

and

$$
\gamma_{\infty}=\lim _{R \rightarrow \infty} \limsup _{n \rightarrow \infty} \int_{|x|>R} \frac{u_{n}^{2}}{|x|^{2}} d x .
$$

Similarly, the concentration at infinity of the sequence $\left\{v_{n}\right\}_{n \in \mathbb{N}}$ is described by quantities $\bar{\rho}_{\infty}, \bar{\mu}_{\infty}$, and $\bar{\gamma}_{\infty}$ defined in an analogous way. We claim that

$$
\mathcal{J} \text { is finite and, for } j \in \mathcal{J} \text {, either } \rho_{j}=0 \text { or } \rho_{j} \geq S^{N / 2} .
$$


For $\varepsilon>0$, let $\phi_{j}^{\varepsilon}$ be a smooth cut-off function centered at $x_{j}, 0 \leq \phi_{j}^{\varepsilon}(x) \leq 1$, such that

$$
\phi_{j}^{\varepsilon}(x)=\left\{\begin{array}{ll}
1, & \text { if }\left|x-x_{j}\right| \leq \varepsilon / 2, \\
0, & \text { if }\left|x-x_{j}\right| \geq \varepsilon,
\end{array} \quad \text { and } \quad\left|\nabla \phi_{j}^{\varepsilon}(x)\right| \leq \frac{4}{\varepsilon} \quad \text { for all } x \in \mathbb{R}^{N} .\right.
$$

Testing $J_{\nu}^{\prime}\left(u_{n}, v_{n}\right)$ with $\left(u_{n} \phi_{j}^{\varepsilon}, 0\right)$ we obtain

$$
\begin{aligned}
0 & =\lim _{n \rightarrow \infty}\left\langle J_{\nu}^{\prime}\left(u_{n}, v_{n}\right),\left(u_{n} \phi_{j}^{\varepsilon}, 0\right)\right\rangle \\
& =\lim _{n \rightarrow \infty}\left(\int_{\mathbb{R}^{N}}\left[\left|\nabla u_{n}\right|^{2} \phi_{j}^{\varepsilon}+u_{n} \nabla u_{n} \cdot \nabla \phi_{j}^{\varepsilon}-\lambda_{1} \frac{u_{n}^{2} \phi_{j}^{\varepsilon}}{|x|^{2}}-\phi_{j}^{\varepsilon}\left|u_{n}\right|^{2^{*}}-\alpha \nu h(x)\left|u_{n}\right|^{\alpha}\left|v_{n}\right|^{\beta} \phi_{j}^{\varepsilon}\right] d x\right) .
\end{aligned}
$$

From $(\mathbf{H})$ and since $0 \notin \operatorname{supp}\left(\phi_{j}^{\varepsilon}\right)$ provided $\varepsilon$ is small enough, letting $\varepsilon \rightarrow 0$ we obtain that $\mu_{j}-\rho_{j} \leq 0$. Since $S \rho_{j}^{2 / 2^{*}} \leq \mu_{j}$, we conclude that, for all $j \in \mathcal{J}$, either $\rho_{j}=0$ or $\rho_{j} \geq S^{N / 2}$, which implies that $\mathcal{J}$ is finite. The claim is thereby proved. Notice that, by an analogous argument, the same conclusion holds for $\overline{\rho_{k}}$ and $\mathcal{K}$, i.e.

$$
\mathcal{K} \text { is finite, and, for } k \in \mathcal{K} \text {, either } \bar{\rho}_{k}=0 \text { or } \bar{\rho}_{k} \geq S^{N / 2} \text {. }
$$

To analyze the concentration at 0 , we follow closely the argument used in [1]. By choosing suitable cut-off functions centered at the origin, we can easily prove that $\mu_{0}-\lambda_{1} \gamma_{0} \leq \rho_{0}$ and $\bar{\mu}_{0}-\lambda_{2} \bar{\gamma}_{0} \leq \bar{\rho}_{0}$. On the other hand, using the definition of $S\left(\lambda_{1}\right)$ and $S\left(\lambda_{2}\right)$, we obtain that

$$
\mu_{0}-\lambda_{1} \gamma_{0} \geq S\left(\lambda_{1}\right) \rho_{0}^{2 / 2^{*}} \quad \text { and } \quad \bar{\mu}_{0}-\lambda_{2} \bar{\gamma}_{0} \geq S\left(\lambda_{2}\right) \bar{\rho}_{0}^{2 / 2^{*}} .
$$

It follows that

$$
\text { either } \rho_{0}=0 \text { or } \rho_{0} \geq S^{\frac{N}{2}}\left(\lambda_{1}\right)
$$

and

$$
\text { either } \bar{\rho}_{0}=0 \text { or } \bar{\rho}_{0} \geq S^{\frac{N}{2}}\left(\lambda_{2}\right) \text {. }
$$

By choosing a suitable cut-off function supported in a neighborhood of $\infty$, we can analogously prove that

$$
\begin{gathered}
\mu_{\infty}-\lambda_{1} \gamma_{\infty} \geq S\left(\lambda_{1}\right) \rho_{\infty}^{2 / 2^{*}} \text { and } \bar{\mu}_{\infty}-\lambda_{2} \bar{\gamma}_{\infty} \geq S\left(\lambda_{2}\right) \bar{\rho}_{\infty}^{2 / 2^{*}}, \\
\text { either } \rho_{\infty}=0 \text { or } \rho_{\infty} \geq S^{\frac{N}{2}}\left(\lambda_{1}\right),
\end{gathered}
$$

and

$$
\text { either } \bar{\rho}_{\infty}=0 \text { or } \bar{\rho}_{\infty} \geq S^{\frac{N}{2}}\left(\lambda_{2}\right) \text {. }
$$

From (25) we have that

$$
c=\left(\frac{1}{2}-\frac{1}{\alpha+\beta}\right)\left\|\left(u_{n}, v_{n}\right)\right\|_{\mathbb{D}}^{2}+\left(\frac{1}{\alpha+\beta}-\frac{1}{2^{*}}\right)\left(\int_{\mathbb{R}^{N}}\left|u_{n}\right|^{2^{*}} d x+\int_{\mathbb{R}^{N}}\left|v_{n}\right|^{2^{*}} d x\right)+o(1)
$$


as $n \rightarrow+\infty$. Letting $n \rightarrow+\infty$, from (26), (27), (28), (33), and (36), we obtain

$$
\begin{gathered}
c \geq\left(\frac{1}{2}-\frac{1}{\alpha+\beta}\right)\left[\left\|\left(u_{0}, v_{0}\right)\right\|_{\mathbb{D}}^{2}+\sum_{j \in \mathcal{J}} \mu_{j}+\left(\mu_{0}-\lambda_{1} \gamma_{0}\right)+\left(\mu_{\infty}-\lambda_{1} \gamma_{\infty}\right)\right. \\
\left.+\sum_{k \in \mathcal{K}} \bar{\mu}_{k}+\left(\bar{\mu}_{0}-\lambda_{2} \bar{\gamma}_{0}\right)+\left(\bar{\mu}_{\infty}-\lambda_{2} \bar{\gamma}_{\infty}\right)\right] \\
+\left(\frac{1}{\alpha+\beta}-\frac{1}{2^{*}}\right)\left[\int_{\mathbb{R}^{N}}\left|u_{0}\right|^{2^{*}}+\int_{\mathbb{R}^{N}}\left|v_{0}\right|^{2^{*}}+\sum_{j \in \mathcal{J}} \rho_{j}+\sum_{k \in \mathcal{K}} \bar{\rho}_{k}+\rho_{0}+\bar{\rho}_{0}+\rho_{\infty}+\bar{\rho}_{\infty}\right] \\
\geq\left(\frac{1}{2}-\frac{1}{\alpha+\beta}\right)\left[S\left(\sum_{j \in \mathcal{J}} \rho_{j}^{2 / 2^{*}}+\sum_{k \in \mathcal{K}} \bar{\rho}_{k}^{2 / 2^{*}}\right)+S\left(\lambda_{1}\right)\left(\rho_{0}^{2 / 2^{*}}+\rho_{\infty}^{2 / 2^{*}}\right)+S\left(\lambda_{2}\right)\left(\bar{\rho}_{0}^{2 / 2^{*}}+\bar{\rho}_{\infty}^{2 / 2^{*}}\right)\right] \\
+\left(\frac{1}{\alpha+\beta}-\frac{1}{2^{*}}\right)\left[\sum_{j \in \mathcal{J}} \rho_{j}+\sum_{k \in \mathcal{K}} \bar{\rho}_{k}+\rho_{0}+\bar{\rho}_{0}+\rho_{\infty}+\bar{\rho}_{\infty}\right] .
\end{gathered}
$$

If $\rho_{j} \neq 0$, from above and (31), we obtain

$$
c \geq\left(\frac{1}{2}-\frac{1}{\alpha+\beta}\right) S^{1+\frac{N}{2} \frac{2}{2^{*}}}+\left(\frac{1}{\alpha+\beta}-\frac{1}{2^{*}}\right) S^{\frac{N}{2}}=\frac{1}{N} S^{N / 2},
$$

thus giving rise to a contradiction with the hypothesis on $c$. Hence $\mu_{j}=\rho_{j}=0$ for all $j \in \mathcal{J}$. In the same way, using (32), we can prove that $\bar{\mu}_{k}=\bar{\rho}_{k}=0$ for all $k \in \mathcal{K}$. If $\rho_{0} \neq 0$, from (39) and (34), we obtain

$$
c \geq \frac{1}{N} S^{N / 2}\left(\lambda_{1}\right)
$$

thus contradicting the hypothesis on $c$. Hence $\rho_{0}=0$. In the same way, using (35), we can prove that $\bar{\rho}_{0}=0$. Analogously, from $(37-38)$ it follows that $\rho_{\infty}=\bar{\rho}_{\infty}=0$. Hence $\left\{\left(u_{n}, v_{n}\right)\right\}_{n \in \mathbb{N}}$ converges strongly to $\left(u_{0}, v_{0}\right)$ in $L^{2^{*}}\left(\mathbb{R}^{N}\right) \times L^{2^{*}}\left(\mathbb{R}^{N}\right)$. To conclude, it is now enough to observe that

$$
\left\|\left(u_{n}, v_{n}\right)-\left(u_{0}, v_{0}\right)\right\|_{\mathbb{D}}^{2}=\left\langle J_{\nu}^{\prime}\left(u_{n}, v_{n}\right),\left(u_{n}-u_{0}, v_{n}-v_{0}\right)\right\rangle+o(1)=o(1) \text { as } n \rightarrow+\infty .
$$

Hence $\left\{\left(u_{n}, v_{n}\right)\right\}_{n \in \mathbb{N}}$ converges strongly to $\left(u_{0}, v_{0}\right)$ in $\mathbb{D}$.

Notice that the energy level below which the Palais-Smale condition holds provided by Lemma 3.2 is independent of the parameter $\nu$. If $\nu$ is sufficiently small, the above Palais-Smale condition can be extended to higher energy levels. To obtain such an improvement, we first observe that, for $\alpha, \beta>2$ and $\nu$ small enough, any ground state solution to (1) must be necessarily semi-trivial. To prove this, we need the following algebraic lemma.

Lemma 3.3. Let $A, B>0$, and $\theta \geq 2$ be fixed. For any $\nu>0$, let

$$
S_{\nu}=\left\{\sigma \in \mathbb{R}^{+} \mid A \sigma^{\frac{N-2}{N}} \leq \sigma+B \nu \sigma^{\frac{\theta}{2} \frac{N-2}{N}}\right\} .
$$

Then, for all $\varepsilon>0$, there exists $\nu_{1}>0$ depending only on $\varepsilon, A, B, \theta$, and $N$, such that

$$
\inf S_{\nu} \geq(1-\varepsilon) A^{\frac{N}{2}} \quad \text { for all } 0<\nu<\nu_{1}
$$

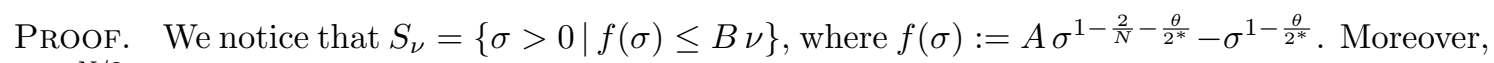
$f\left(A^{N / 2}\right)=0$. If $\theta \leq 2^{*}$, then $f$ is a strictly decreasing function. If $\theta>2^{*}$, then $f$ has a global negative minimum at $\sigma=\left(\frac{A(\theta-2)}{\theta-2^{*}}\right)^{N / 2}>A^{N / 2}$ and tends to 0 as $\sigma \rightarrow+\infty$. In any case, $f$ is strictly decreasing in $\left(0, A^{N / 2}\right]$, has only one zero at $A^{N / 2}, \lim _{\sigma \rightarrow 0^{+}} f(\sigma)=+\infty$, and $f(\sigma)<0$ in $\left(A^{N / 2},+\infty\right)$. Hence inf $S_{\nu}=f^{-1}(B \nu) \rightarrow A^{N / 2}$ as $\nu \rightarrow 0^{+}$and the conclusion follows. 
The following theorem ensures that, when $\nu$ is sufficiently small and $\alpha, \beta \geq 2$, all ground states solutions are semi-trivial.

Theorem 3.4. Assume that $\alpha, \beta \geq 2$, then there exists $\nu_{1}>0$ such that for all $0<\nu \leq \nu_{1}$

$$
c^{*}(\nu)=\inf _{\mathcal{N}_{\nu}} J_{\nu}(u, v)= \begin{cases}\frac{1}{N} S^{\frac{N}{2}}\left(\lambda_{2}\right), & \text { if } \lambda_{1} \leq \lambda_{2} \\ \frac{1}{N} S^{\frac{N}{2}}\left(\lambda_{1}\right), & \text { if } \lambda_{1}>\lambda_{2}\end{cases}
$$

Moreover $c^{*}(\nu)$ is achieved by and only by

$$
\left\{\begin{array}{l}
\left(0, \pm z_{\mu}^{\lambda_{2}}\right), \quad \mu>0, \text { if } \lambda_{1}<\lambda_{2}, \\
\left( \pm z_{\mu}^{\lambda_{1}}, 0\right), \quad \mu>0, \quad \text { if } \lambda_{2}<\lambda_{1}, \\
\left(0, \pm z_{\mu}^{\lambda_{2}}\right), \quad\left( \pm z_{\mu}^{\lambda_{1}}, 0\right), \quad \mu>0, \quad \text { if } \lambda_{1}=\lambda_{2} .
\end{array}\right.
$$

Proof. Let us prove the theorem in the case $\lambda_{1} \leq \lambda_{2}$. Since $\left(0, z_{1}^{\lambda_{2}}\right) \in \mathcal{N}_{\nu}$, then, for all $\nu>0, c^{*}(\nu) \leq J_{\nu}\left(0, z_{1}^{\lambda_{2}}\right)=\frac{1}{N} S^{\frac{N}{2}}\left(\lambda_{2}\right)$. Assume by contradiction that $c^{*}\left(\nu_{n}\right)<\frac{1}{N} S^{\frac{N}{2}}\left(\lambda_{2}\right)$ for some sequence $\nu_{n} \rightarrow 0^{+}$. Since $\lambda_{1} \leq \lambda_{2}$, there results that $c^{*}\left(\nu_{n}\right)<\frac{1}{N}\left(\min \left\{S\left(\lambda_{1}\right), S\left(\lambda_{2}\right)\right\}\right)^{N / 2}$. By Lemmas 3.1 and 3.2, the Palais-Smale condition holds at level $c^{*}\left(\nu_{n}\right)$ on the Nehari manifold, hence $c^{*}\left(\nu_{n}\right)$ is achieved by some $\left(\phi_{n}, \psi_{n}\right) \in \mathcal{N}_{\nu}$. Since also $\left(\left|\phi_{n}\right|,\left|\psi_{n}\right|\right) \in \mathcal{N}_{\nu}$ and $J_{\nu}\left(\phi_{n}, \psi_{n}\right)=$ $J_{\nu}\left(\left|\phi_{n}\right|,\left|\psi_{n}\right|\right)$, we have that also $\left(\left|\phi_{n}\right|,\left|\psi_{n}\right|\right)$ is a ground state for $J_{\nu}$. Hence we can assume that $\phi_{n} \geq 0$ and $\psi_{n} \geq 0$. Moreover, by classical regularity results, $\phi_{n}$ and $\psi_{n}$ are smooth in $\mathbb{R}^{N} \backslash\{0\}$. Moreover, $\phi_{n} \not \equiv 0$ and $\psi_{n} \not \equiv 0$ for all $n \in \mathbb{N}$. Indeed, assume by contradiction that $\psi_{n} \equiv 0$ for some $n$, then $\phi_{n} \not \equiv 0$ solves

$$
-\Delta \phi_{n}-\lambda_{1} \frac{\phi_{n}}{|x|^{2}}=\phi_{n}^{2^{*}-1}
$$

Hence, by [28], $\phi_{n}=z_{\mu_{n}}^{\lambda_{1}}$ for some $\mu_{n}>0$. Therefore, (11) yields that

$$
c^{*}\left(\nu_{n}\right)=J_{\nu}\left(\phi_{n}, 0\right)=I_{\lambda_{1}}\left(\phi_{n}\right)=\frac{1}{N} S^{\frac{N}{2}}\left(\lambda_{1}\right),
$$

thus giving rise to a contradiction with the fact that $c^{*}\left(\nu_{n}\right)<\frac{1}{N}\left(\min \left\{S\left(\lambda_{1}\right), S\left(\lambda_{2}\right)\right\}\right)^{N / 2}$. In the same way, we can prove that $\phi_{n} \not \equiv 0$ for all $n \in \mathbb{N}$. The maximum principle in $\mathbb{R}^{N} \backslash\{0\}$ implies that $\phi_{n}>0$ and $\psi_{n}>0$ in $\mathbb{R}^{N} \backslash\{0\}$ for all $n \in \mathbb{N}$. Notice that

$$
c^{*}\left(\nu_{n}\right)=J_{\nu_{n}}\left(\phi_{n}, \psi_{n}\right)=\frac{1}{N}\left(\int_{\mathbb{R}^{N}} \phi_{n}^{2^{*}} d x+\int_{\mathbb{R}^{N}} \psi_{n}^{2^{*}} d x\right)+\nu(\alpha+\beta)\left(\frac{1}{2}-\frac{1}{\alpha+\beta}\right) \int_{\mathbb{R}^{N}} h(x) \phi_{n}^{\alpha} \psi_{n}^{\beta} d x,
$$

then, by setting $\sigma_{1}^{n}:=\int_{\mathbb{R}^{N}} \phi_{n}^{2^{*}} d x$ and $\sigma_{2}^{n}:=\int_{\mathbb{R}^{N}} \psi_{n}^{2^{*}}$, we obtain that $\sigma_{1}^{n}>0, \sigma_{2}^{n}>0$,

$$
\max \left\{\sigma_{1}^{n}, \sigma_{2}^{n}\right\}<S^{\frac{N}{2}}\left(\lambda_{2}\right), \text { and } \sigma_{1}^{n}+\sigma_{2}^{n}<S^{\frac{N}{2}}\left(\lambda_{2}\right) .
$$

Since $\left(\phi_{n}, \psi_{n}\right)$ are solutions to system (1) with $\nu=\nu_{n}$, we obtain that

$$
S\left(\lambda_{1}\right)\left(\sigma_{1}^{n}\right)^{\frac{2}{2^{*}}} \leq \sigma_{1}^{n}+\nu_{n} \alpha \int_{\mathbb{R}^{N}} h(x) \phi_{n}^{\alpha} \psi_{n}^{\beta} d x
$$

and

Since

$$
S\left(\lambda_{2}\right)\left(\sigma_{2}^{n}\right)^{\frac{2}{2^{*}}} \leq \sigma_{2}^{n}+\nu_{n} \beta \int_{\mathbb{R}^{N}} h(x) \phi_{n}^{\alpha} \psi_{n}^{\beta} d x
$$

$$
\int_{\mathbb{R}^{N}} h(x) \phi_{n}^{\alpha} \psi_{n}^{\beta} d x \leq C(h)\left(\int_{\mathbb{R}^{N}} \phi_{n}^{2^{*}} d x\right)^{\frac{\alpha}{2^{*}}}\left(\int_{\mathbb{R}^{N}} \psi_{n}^{2^{*}} d x\right)^{\frac{\beta}{2^{*}}}
$$


then, using (40) and the fact that $S\left(\lambda_{2}\right) \leq S\left(\lambda_{1}\right)$, we conclude that

$$
S\left(\lambda_{2}\right)\left(\sigma_{1}^{n}\right)^{\frac{2}{2^{*}}} \leq \sigma_{1}^{n}+\alpha C(h)\left(S\left(\lambda_{2}\right)\right)^{\frac{\beta(N-2)}{4}} \nu_{n}\left(\sigma_{1}^{n}\right)^{\frac{\alpha}{2^{*}}}
$$

and

$$
S\left(\lambda_{2}\right)\left(\sigma_{2}^{n}\right)^{\frac{2}{2^{*}}} \leq \sigma_{2}^{n}+\beta C(h)\left(S\left(\lambda_{2}\right)\right)^{\frac{\alpha(N-2)}{4}} \nu_{n}\left(\sigma_{2}^{n}\right)^{\frac{\beta}{2^{*}}} .
$$

Let us fix $\varepsilon>0$ such that $2-2 \varepsilon>\frac{3}{2}$. Since $\alpha, \beta \geq 2$, we can use Lemma 3.3 to conclude that, for $n$ sufficiently large,

$$
\sigma_{1}^{n} \geq(1-\varepsilon) S^{\frac{N}{2}}\left(\lambda_{2}\right) \text { and } \sigma_{2}^{n} \geq(1-\varepsilon) S^{\frac{N}{2}}\left(\lambda_{2}\right) .
$$

Then

$$
\sigma_{1}^{n}+\sigma_{2}^{n} \geq(2-2 \varepsilon) S^{\frac{N}{2}}\left(\lambda_{2}\right)>\frac{3}{2} S^{\frac{N}{2}}\left(\lambda_{2}\right)
$$

a contradiction with (40). Therefore $c^{*}(\nu)=\frac{1}{N} S^{\frac{N}{2}}\left(\lambda_{2}\right)$ provided $\nu$ is sufficiently small. Assume now that $(\phi, \psi)$ is a minimizer attaining $c^{*}(\nu)=\frac{1}{N} S^{\frac{N}{2}}\left(\lambda_{2}\right)$ for $\nu$ small. Repeating the above argument, we can easily prove that either $\phi \equiv 0$ or $\psi \equiv 0$. If $\lambda_{1}<\lambda_{2}$, then necessarily $\phi \equiv 0$. If not, then $\psi \equiv 0$ and $\phi \not \equiv 0$ weakly solves

$$
-\Delta \phi-\lambda_{1} \frac{\phi}{|x|^{2}}=|\phi|^{2^{*}-2} \phi .
$$

We notice that $\phi$ does not change sign. Indeed $\phi_{+}=\max \{\phi, 0\}, \phi_{-}=\max \{-\phi, 0\} \in N_{\lambda_{1}}$ (see $(13))$, hence $\left(\phi_{+}, 0\right),\left(\phi_{-}, 0\right) \in \mathcal{N}_{\nu}$. If both $\phi_{+} \not \equiv 0$ and $\phi_{-} \not \equiv 0$, then

$$
c^{*}(\nu)=J_{\nu}(\phi, 0)=\frac{1}{N} \int_{\mathbb{R}^{N}}|\phi|^{2^{*}} d x=\frac{1}{N} \int_{\mathbb{R}^{N}} \phi_{+}^{2^{*}} d x+\frac{1}{N} \int_{\mathbb{R}^{N}} \phi_{-}^{2^{*}} d x>J_{\nu}\left(\phi_{+}, 0\right) \geq c^{*}(\nu),
$$

a contradiction. Hence $\phi$ does not change sign and, by [28], $\phi= \pm z_{\mu}^{\lambda_{1}}$ for some $\mu>0$. Then

$$
c^{*}(\nu)=J_{\nu}(\phi, 0)=\frac{1}{N} S^{\frac{N}{2}}\left(\lambda_{1}\right)>\frac{1}{N} S^{\frac{N}{2}}\left(\lambda_{2}\right),
$$

which is a contradiction. Hence $\phi \equiv 0$ and any minimizer in this case has necessarily the form $(0, \psi)$, where $\psi \not \equiv 0$ weakly solves

$$
-\Delta \psi-\lambda_{2} \frac{\psi}{|x|^{2}}=|\psi|^{2^{*}-2} \psi
$$

Arguing as above, it is easy to verify that $\psi$ does not change sign, and then, by [28], $\psi(x)= \pm z_{\mu}^{\lambda_{2}}$ for some $\mu>0$ and the conclusion follows. In the case $\lambda_{1}=\lambda_{2}$, the couples $\left( \pm z_{\mu}^{\lambda_{1}}, 0\right)$ and $\left(0, \pm z_{\mu}^{\lambda_{2}}\right)$, $\mu>0$, completely describe the family of minimizers.

In order to find positive mountain pass solutions to (1), it is convenient to consider, under the same hypotheses on $h, \lambda_{1}, \lambda_{2}$ and $\nu$, the following modified problem

$$
\left\{\begin{array}{l}
-\Delta u-\lambda_{1} \frac{u}{|x|^{2}}=u_{+}^{2^{*}-1}+\nu h(x) \alpha u_{+}^{\alpha-1} v_{+}^{\beta}, \quad \text { in } \mathbb{R}^{N}, \\
-\Delta v-\lambda_{2} \frac{v}{|x|^{2}}=v_{+}^{2^{*}-1}+\nu h(x) \beta u_{+}^{\alpha} v_{+}^{\beta-1}, \quad \text { in } \mathbb{R}^{N}
\end{array}\right.
$$

where $u_{+}=\max \{u, 0\}$. Weak $\mathcal{D}^{1,2}\left(\mathbb{R}^{N}\right)$-solutions to problem (41) are critical points of the functional

$$
\bar{J}_{\nu}: \mathbb{D} \rightarrow \mathbb{R}, \quad \bar{J}_{\nu}(u, v)=\bar{I}_{\lambda_{1}}(u)+\bar{I}_{\lambda_{2}}(v)-\nu \int_{\mathbb{R}^{N}} h(x) u_{+}^{\alpha} v_{+}^{\beta} d x
$$


where

$$
\bar{I}_{\lambda_{i}}(w)=\frac{1}{2} Q_{\lambda_{i}}(w)-\frac{1}{2^{*}} \int_{\mathbb{R}^{N}} w_{+}^{2^{*}} d x, \quad i=1,2
$$

If $(u, v)$ is a critical point of $\bar{J}_{\nu}$, then, by testing with $u_{-}=\max \{-u, 0\}$ and $v_{-}=\max \{-v, 0\}$, we easily obtain that $u \geq 0$ and $v \geq 0$. Hence critical points of $\bar{J}_{\nu}$ provide positive solutions to the original problem (1). We denote by $\overline{\mathcal{N}}_{\nu}$ the Nehari manifold associated to $\bar{J}_{\nu}$, i.e.

$$
\overline{\mathcal{N}}_{\nu}=\left\{(u, v) \in \mathbb{D} \backslash\{(0,0)\}:\left\langle\left(\bar{J}_{\nu}\right)^{\prime}(u, v),(u, v)\right\rangle=0\right\} .
$$

The following lemma provides, for $\nu$ small and $\alpha, \beta \geq 2$, an improved Palais-Smale condition for $\bar{J}_{\nu}$ above the critical threshold of Lemma 3.2 .

Lemma 3.5. Let $\mathbf{( H ) ~ h o l d ~ a n d ~} \alpha, \beta \geq 2$ satisfy (21). If $0<\lambda_{1} \leq \lambda_{2}<(N-2)^{2} / 4$ and

$$
S^{N / 2}\left(\lambda_{1}\right)+S^{N / 2}\left(\lambda_{2}\right)<S^{N / 2}
$$

then there exists $\nu_{1}>0$ (depending only on $N, \lambda_{1}, \lambda_{2}, \alpha, \beta$, and $h$ ) such that if $\nu \leq \nu_{1}$ and $\left\{\left(u_{n}, v_{n}\right)\right\}_{n \in \mathbb{N}} \subset \mathbb{D}$ is a Palais-Smale sequence for $\bar{J}_{\nu}$ at level $c \in \mathbb{R}$ with c satisfying

$$
\frac{1}{N} S^{N / 2}\left(\lambda_{1}\right)<c<\frac{1}{N}\left(S^{N / 2}\left(\lambda_{1}\right)+S^{N / 2}\left(\lambda_{2}\right)\right)
$$

and

$$
c \neq \frac{\ell}{N} S^{N / 2}\left(\lambda_{2}\right) \text { for all } \ell \in \mathbb{N} \backslash\{0\},
$$

then $\left\{\left(u_{n}, v_{n}\right)\right\}_{n \in \mathbb{N}}$ admits a subsequence strongly converging in $\mathbb{D}$.

Proof. Arguing as in the proof of Lemma 3.2, it is easy to verify that $\left\{\left(u_{n}, v_{n}\right)\right\}_{n \in \mathbb{N}}$ is bounded in $\mathbb{D}$ and admits a subsequence, still denoted as $\left\{\left(u_{n}, v_{n}\right)\right\}_{n \in \mathbb{N}}$, weakly converging to some $\left(u_{0}, v_{0}\right) \in \mathbb{D}$. We claim that $\left(\left(u_{n}\right)_{-},\left(v_{n}\right)_{-}\right) \rightarrow(0,0)$ strongly in $\mathbb{D}$. Indeed $\left(\bar{J}_{\nu}\right)^{\prime}\left(u_{n}, v_{n}\right) \rightarrow 0$ strongly in $\mathbb{D}$ implies

$$
\left\langle\left(\bar{J}_{\nu}\right)^{\prime}\left(u_{n}, v_{n}\right),\left(\left(u_{n}\right)_{-}, 0\right)\right\rangle=-\int_{\mathbb{R}^{N}}\left|\nabla\left(u_{n}\right)_{-}\right|^{2}+\lambda_{1} \int_{\mathbb{R}^{N}} \frac{\left(u_{n}\right)_{-}^{2}}{|x|^{2}} d x=o(1) \quad \text { as } n \rightarrow \infty .
$$

Thus $\left(u_{n}\right)_{-} \rightarrow 0$ strongly in $\mathcal{D}^{1,2}\left(\mathbb{R}^{N}\right)$. The same holds for $\left(v_{n}\right)_{-}$. Hence $\left(\left(u_{n}\right)_{+},\left(v_{n}\right)_{+}\right)$is a bounded Palais-Smale sequence of $\bar{J}_{\nu}$, and, having positive components, of $J_{\nu}$. Therefore, without loss of generality, we can assume directly that

$$
u_{n} \geq 0, \quad v_{n} \geq 0, \quad \text { and }\left\{\left(u_{n}, v_{n}\right)\right\}_{n \in \mathbb{N}} \text { is Palais-Smale sequence for } J_{\nu} \text { at level } c .
$$

From the Concentration Compactness Principle [17, 18] and the same arguments as in the proof of Lemma 3.2, we deduce the existence of a subsequence, still denoted as $\left\{\left(u_{n}, v_{n}\right)\right\}_{n \in \mathbb{N}}$, two at most countable sets $\mathcal{J}$ and $\mathcal{K}$, sets of points $\left\{x_{j} \in \mathbb{R}^{N} \backslash\{0\}, j \in \mathcal{J}\right\}$ and $\left\{y_{k} \in \mathbb{R}^{N} \backslash\{0\}, k \in \mathcal{K}\right\}$, real numbers $\mu_{j}, \rho_{j}, j \in \mathcal{J}, \bar{\mu}_{k}, \bar{\rho}_{k}, k \in \mathcal{K}, \mu_{0}, \rho_{0}, \gamma_{0}, \bar{\mu}_{0}, \bar{\rho}_{0}$, and $\bar{\gamma}_{0}$, such that (26-35) hold. Moreover, defining $\rho_{\infty}, \mu_{\infty}$, and $\gamma_{\infty}$ as in (29-30) (and analogously $\bar{\rho}_{\infty}, \bar{\mu}_{\infty}$, and $\bar{\gamma}_{\infty}$ for the concentration of $v_{n}$ at $\left.\infty\right),(37)$ and $(38)$ are satisfied.

Claim 1. We claim that either $u_{n} \rightarrow u_{0}$ strongly in $L^{2^{*}}\left(\mathbb{R}^{N}\right)$ or $v_{n} \rightarrow v_{0}$ strongly in $L^{2^{*}}\left(\mathbb{R}^{N}\right)$. Assume by contradiction that the $L^{2^{*}}\left(\mathbb{R}^{N}\right)$-convergence of neither $u_{n}$ nor $v_{n}$ is strong. Hence there exist $j_{0} \in \mathcal{J} \cup\{0\} \cup\{\infty\}$ and $k_{0} \in \mathcal{K} \cup\{0\} \cup\{\infty\}$ such that $\rho_{j_{0}}>0$ and $\bar{\rho}_{k_{0}}>0$. From (39), (31), 
(32), (34), (35), (37), and (38), we obtain that

$$
\begin{aligned}
c=J_{\nu}\left(u_{n}, v_{n}\right)+o(1) & \geq\left(\frac{1}{2}-\frac{1}{\alpha+\beta}\right)\left(S\left(\lambda_{1}\right) \rho_{j_{0}}^{2 / 2^{*}}+S\left(\lambda_{2}\right) \bar{\rho}_{k_{0}}^{2 / 2^{*}}\right)+\left(\frac{1}{\alpha+\beta}-\frac{1}{2^{*}}\right)\left(\rho_{j_{0}}+\bar{\rho}_{k_{0}}\right) \\
& \geq \frac{1}{N}\left(S^{N / 2}\left(\lambda_{1}\right)+S^{N / 2}\left(\lambda_{2}\right)\right)
\end{aligned}
$$

which is in contradiction with (45).

Claim 2. We claim that either $u_{n} \rightarrow u_{0}$ strongly in $\mathcal{D}^{1,2}\left(\mathbb{R}^{N}\right)$ or $v_{n} \rightarrow v_{0}$ strongly in $\mathcal{D}^{1,2}\left(\mathbb{R}^{N}\right)$. Indeed, assume that $u_{n}$ converges to $u_{0}$ strongly in $L^{2^{*}}\left(\mathbb{R}^{N}\right)$. Then

$$
\left\|u_{n}-u_{0}\right\|_{\lambda_{1}}^{2}=\left\langle J_{\nu}^{\prime}\left(u_{n}, v_{n}\right),\left(u_{n}-u_{0}, 0\right)\right\rangle+o(1)=o(1) \text { as } n \rightarrow+\infty .
$$

Hence $u_{n}$ converges strongly to $u_{0}$ in $\mathcal{D}^{1,2}\left(\mathbb{R}^{N}\right)$. The same argument yields the claim when $v_{n}$ converges to $v_{0}$ strongly in $L^{2^{*}}\left(\mathbb{R}^{N}\right)$.

In view of the above claims, we distinguish two cases.

Case 1. $v_{n}$ converges strongly to $v_{0}$ in $\mathcal{D}^{1,2}\left(\mathbb{R}^{N}\right)$. In this case it is easy to prove that $\mathcal{J} \cup\{0\} \cup\{\infty\}$ is reduced to be at most a singleton set: indeed, if $\mathcal{J} \cup\{0\} \cup\{\infty\}$ contains more than one point, (39), (31), (32), (34), (35), (37), and (38) yield that

$$
c \geq \frac{2}{N} S^{N / 2}\left(\lambda_{1}\right) \geq \frac{1}{N}\left(S^{N / 2}\left(\lambda_{1}\right)+S^{N / 2}\left(\lambda_{2}\right)\right)
$$

thus contradicting (45). Hence $\mathcal{J} \cup\{0\} \cup\{\infty\}$ is at most a singleton set. In order to prove that also $u_{n}$ converges strongly in $\mathcal{D}^{1,2}\left(\mathbb{R}^{N}\right)$ (up to a subsequence), we argue by contradiction and assume that $u_{n}$ converges weakly but none of its subsequences converges strongly to $u_{0}$. We claim that $v_{0} \not \equiv 0$. Indeed, if, by contradiction, $v_{0} \equiv 0$ and $u_{0} \not \equiv 0$, we have that $u_{0}$ is a positive weak $\mathcal{D}^{1,2}\left(\mathbb{R}^{N}\right)$-solution to

$$
-\Delta u_{0}-\lambda_{1} \frac{u_{0}}{|x|^{2}}-u_{0}^{2^{*}-1}=0 .
$$

Hence $u_{0}=z_{\mu}^{\lambda_{1}}$ for some $\mu>0$ and $\int_{\mathbb{R}^{N}}\left|u_{0}\right|^{2^{*}}=S^{N / 2}\left(\lambda_{1}\right)$. Hence from (39) and (31-38), taking into account the definition of $S\left(\lambda_{1}\right)$ and the fact that $u_{n}$ concentrates exactly at one point, we deduce that

$$
c \geq \frac{1}{N}\left(\int_{\mathbb{R}^{N}} u_{0}^{2^{*}} d x+S^{\frac{N}{2}}\left(\lambda_{1}\right)\right) \geq \frac{2}{N} S^{\frac{N}{2}}\left(\lambda_{1}\right),
$$

in contradiction with (45) and the fact that $S\left(\lambda_{2}\right) \leq S\left(\lambda_{1}\right)$. On the other hand, if $v_{0} \equiv 0$ and $u_{0} \equiv 0$, then $u_{n}$ solves

$$
-\Delta u_{n}-\lambda_{1} \frac{u_{n}}{|x|^{2}}-u_{n}^{2^{*}-1}=o(1), \quad \text { in the dual space }\left(\mathcal{D}^{1,2}\left(\mathbb{R}^{N}\right)\right)^{\star} .
$$

Since the concentration of $u_{n}$ occurs at exactly one point, there exists $j \in \mathcal{J} \cup\{0, \infty\}$ such that

$$
c=J_{\nu}\left(u_{n}, v_{n}\right)+o(1)=\frac{1}{N} \int_{\mathbb{R}^{N}} u_{n}^{2^{*}} d x+o(1) \underset{n \rightarrow \infty}{\longrightarrow} \frac{1}{N} \rho_{j} .
$$

If $j \neq 0$ and $j \neq \infty$, then $\left\{u_{n}\right\}_{n \in \mathbb{N}}$ is a positive Palais-Smale sequence for the functional

$$
I_{0}(u)=\frac{1}{2} \int_{\mathbb{R}^{N}}|\nabla u|^{2} d x-\frac{1}{2^{*}} \int_{\mathbb{R}^{N}} u^{2^{*}} d x, \quad u \in \mathcal{D}^{1,2}\left(\mathbb{R}^{N}\right) .
$$

Hence, in view of the characterization of Palais-Smale sequences of $I_{0}$ proved in [27], we obtain that $\rho_{j}=\ell S^{\frac{N}{2}}$ for some $\ell \in \mathbb{N} \backslash\{0\}$, thus contradicting (44) and (45). In the case where $j \in\{0, \infty\}$, $u_{n}$ is a nonnegative Palais-Smale sequence for the functional $I_{\lambda_{1}}$ defined in (6) at level $c$, then an 
analogous argument as above and the noncompactness analysis performed in [26, Theorem 3.1] imply that

$$
c=J_{\nu}\left(u_{n}, v_{n}\right)+o(1)=I_{\lambda_{1}}\left(u_{n}\right)+o(1) \underset{n \rightarrow \infty}{\longrightarrow} \frac{\ell}{N} S^{\frac{N}{2}}\left(\lambda_{1}\right)
$$

for some $\ell \in \mathbb{N}$. Consequently, a contradiction with assumptions (45) and (46) is reached. Hence we conclude that $v_{0} \not \equiv 0$.

The above discussion allows us to exclude the case $v_{0} \equiv 0$. Thus we may assume that $v_{n}$ converges strongly to $v_{0}$ in $\mathcal{D}^{1,2}\left(\mathbb{R}^{N}\right), v_{0} \not \equiv 0$, and $u_{n}$ converge weakly (and not strongly) to $u_{0}$ in $\mathcal{D}^{1,2}\left(\mathbb{R}^{N}\right)$. If $u_{0} \equiv 0$, then $v_{0}$ weakly solves

$$
-\Delta v_{0}-\lambda_{2} \frac{v_{0}}{|x|^{2}}-v_{0}^{2^{*}-1}=0,
$$

hence $v_{0}=z_{\mu}^{\lambda_{2}}$ for some $\mu>0$ and $\int_{\mathbb{R}^{N}}\left|v_{0}\right|^{2^{*}}=S^{N / 2}\left(\lambda_{2}\right)$. From (39) and concentration of $u_{n}$ at exactly one point, we deduce that

$$
c=J_{\nu}\left(u_{n}, v_{n}\right)+o(1) \geq \frac{1}{N}\left(S^{\frac{N}{2}}\left(\lambda_{1}\right)+S^{\frac{N}{2}}\left(\lambda_{2}\right)\right)
$$

a contradiction with assumption (45). Hence both $u_{0} \not \equiv 0$ and $v_{0} \not \equiv 0$. Letting $n \rightarrow+\infty$ in

$$
\begin{aligned}
c & =J_{\nu}\left(u_{n}, v_{n}\right)-\frac{1}{2}\left\langle J_{\nu}^{\prime}\left(u_{n}, v_{n}\right),\left(u_{n}, v_{n}\right)\right\rangle+o(1) \\
& =\frac{1}{N}\left(\int_{\mathbb{R}^{N}} u_{n}^{2^{*}} d x+\int_{\mathbb{R}^{N}} v_{n}^{2^{*}} d x\right)-\nu\left(1-\frac{\alpha+\beta}{2}\right) \int_{\mathbb{R}^{N}} h(x) u_{n}^{\alpha} v_{n}^{\beta} d x+o(1),
\end{aligned}
$$

we obtain that, for some $j \in \mathcal{J} \cup\{0, \infty\}$,

$$
c=\frac{1}{N}\left(\int_{\mathbb{R}^{N}} u_{0}^{2^{*}} d x+\int_{\mathbb{R}^{N}} v_{0}^{2^{*}} d x+\rho_{j}\right)+\nu \frac{\alpha+\beta-2}{2} \int_{\mathbb{R}^{N}} h(x) u_{0}^{\alpha} v_{0}^{\beta} d x .
$$

On the other hand, letting $n \rightarrow+\infty$ in $\left\langle J_{\nu}^{\prime}\left(u_{n}, v_{n}\right),\left(u_{0}, v_{0}\right)\right\rangle=o(1)$, we obtain that

$$
\left\|\left(u_{0}, v_{0}\right)\right\|_{\mathbb{D}}^{2}=\int_{\mathbb{R}^{N}} u_{0}^{2^{*}} d x+\int_{\mathbb{R}^{N}} v_{0}^{2^{*}} d x+\nu(\alpha+\beta) \int_{\mathbb{R}^{N}} h(x) u_{0}^{\alpha} v_{0}^{\beta} d x,
$$

i.e. $\left(u_{0}, v_{0}\right) \in \mathcal{N}_{\nu}$. Moreover, from (8), (48), (31), (34), (37), and assumption (45), we deduce that

$$
\begin{aligned}
J_{\nu}\left(u_{0}, v_{0}\right) & =\frac{1}{N}\left(\int_{\mathbb{R}^{N}} u_{0}^{2^{*}} d x+\int_{\mathbb{R}^{N}} v_{0}^{2^{*}} d x\right)+\nu \frac{\alpha+\beta-2}{2} \int_{\mathbb{R}^{N}} h(x) u_{0}^{\alpha} v_{0}^{\beta} d x \\
& =c-\frac{\rho_{j}}{N}<\frac{1}{N}\left(S^{N / 2}\left(\lambda_{1}\right)+S^{N / 2}\left(\lambda_{2}\right)\right)-\frac{1}{N} S^{N / 2}\left(\lambda_{1}\right)=\frac{1}{N} S^{N / 2}\left(\lambda_{2}\right) .
\end{aligned}
$$

Hence we conclude that

$$
\min _{\mathcal{N}_{\nu}} J_{\nu} \leq J_{\nu}\left(u_{0}, v_{0}\right)<\frac{1}{N} S^{N / 2}\left(\lambda_{2}\right),
$$

giving rise to a contradiction with Theorem 3.4 if $\nu \leq \nu_{1}$, for $\nu_{1}$ as in Theorem 3.4. Hence, if $v_{n}$ converges strongly, also the convergence of $u_{n}$ must be strong.

Case 2. $u_{n}$ converges strongly to $u_{0}$ in $\mathcal{D}^{1,2}\left(\mathbb{R}^{N}\right)$. In order to prove that $v_{n} \rightarrow v_{0}$ strongly in $\mathcal{D}^{1,2}\left(\mathbb{R}^{N}\right)$, we again argue by contradiction. Assume that $v_{n}$ converges weakly (and not strongly) to $v_{0}$ in $\mathcal{D}^{1,2}\left(\mathbb{R}^{N}\right)$. First we claim that $u_{0} \not \equiv 0$. To prove the claim we argue by contradiction. Assume that $u_{0} \equiv 0$, then is easy to check that $\left\{v_{n}\right\}_{n \in \mathbb{N}}$ is a positive Palais-Smale sequence for 
the functional $I_{\lambda_{2}}$ defined in (6), at the energy level $c=\lim _{n \rightarrow \infty} I_{\lambda_{2}}\left(v_{n}\right)$. Since $v_{n} \rightarrow v_{0}$ weakly in $\mathcal{D}^{1,2}\left(\mathbb{R}^{N}\right)$, then $v_{0}$ is a weak $\mathcal{D}^{1,2}\left(\mathbb{R}^{N}\right)$-solution to

$$
-\Delta v_{0}-\lambda_{2} \frac{v_{0}}{|x|^{2}}-v_{0}^{2^{*}-1}=0 .
$$

By [26] we obtain that, for some $m, \ell \in \mathbb{N}$,

$$
c=\lim _{n \rightarrow \infty} I_{\lambda_{2}}\left(v_{n}\right)=I_{\lambda_{2}}\left(v_{0}\right)+\frac{m}{N} S^{N / 2}+\frac{\ell}{N} S^{N / 2}\left(\lambda_{2}\right)
$$

From (44) and (45), we deduce that $m=0$. If $v_{0} \equiv 0$, then $c=\frac{\ell}{N} S^{N / 2}\left(\lambda_{2}\right)$ for some $\ell \in \mathbb{N}$, a contradiction with (45-46). If $v_{0} \not \equiv 0$, then, from [28], $v_{0}=z_{\mu}^{\lambda_{2}}$ for some $\mu>0$. Then $I_{\lambda_{2}}\left(v_{0}\right)=\frac{1}{N} S^{N / 2}\left(\lambda_{2}\right)$, hence $c=\frac{\ell+1}{N} S^{N / 2}\left(\lambda_{2}\right)$ with $\ell \in \mathbb{N}$, still giving rise to a contradiction with assumption (46). Hence the claim follows and we can assume $u_{0} \not \equiv 0$.

We now claim that $v_{0} \not \equiv 0$. Indeed, if $v_{0} \equiv 0$, then $u_{0}$ weakly solves

$$
-\Delta u_{0}-\lambda_{1} \frac{u_{0}}{|x|^{2}}-u_{0}^{2^{*}-1}=0,
$$

hence, by [28], $u_{0}=z_{\mu}^{\lambda_{1}}$ for some $\mu>0$ and $\int_{\mathbb{R}^{N}}\left|u_{0}\right|^{2^{*}}=S^{N / 2}\left(\lambda_{1}\right)$. Therefore, from (39), (32), (35), and (38), we deduce that $c \geq \frac{1}{N}\left(S^{N / 2}\left(\lambda_{1}\right)+S^{N / 2}\left(\lambda_{2}\right)\right)$, a contradiction with (45). Hence we can assume that $v_{0} \not \equiv 0$. It is clear that $\left(u_{0}, v_{0}\right)$ is a solution to problem (1) and

$$
\begin{aligned}
J_{\nu}\left(u_{0}, v_{0}\right) & =\frac{1}{N}\left(\int_{\mathbb{R}^{N}} u_{0}^{2^{*}} d x+\int_{\mathbb{R}^{N}} v_{0}^{2^{*}} d x\right)+\nu \frac{\alpha+\beta-2}{2} \int_{\mathbb{R}^{N}} h(x) u_{0}^{\alpha} v_{0}^{\beta} d x \\
& \leq c<\frac{1}{N}\left(S^{\frac{N}{2}}\left(\lambda_{1}\right)+S^{\frac{N}{2}}\left(\lambda_{2}\right)\right) .
\end{aligned}
$$

Since $\lambda_{2}<\Lambda_{N}$, then, for some $\varepsilon$ sufficiently small,

$$
\frac{1-\varepsilon}{N}\left(S^{\frac{N}{2}}\left(\lambda_{1}\right)+S^{\frac{N}{2}}\left(\lambda_{2}\right)\right)>\frac{1}{N} S^{\frac{N}{2}}\left(\lambda_{1}\right) .
$$

Fix such an $\varepsilon$. From (47) and the fact that $\left\{v_{n}\right\}_{n \in \mathbb{N}}$ converges weakly and not strongly in $\mathcal{D}^{1,2}\left(\mathbb{R}^{N}\right)$, it follows that at least one of the numbers $\bar{\rho}_{k}, k \in \mathcal{K}, \bar{\rho}_{0}, \bar{\rho}_{\infty}$ is strictly positive and

$$
c=\frac{1}{N}\left(\int_{\mathbb{R}^{N}} u_{0}^{2^{*}} d x+\int_{\mathbb{R}^{N}} v_{0}^{2^{*}} d x+\sum_{k \in \mathcal{K}} \bar{\rho}_{k}+\bar{\rho}_{0}+\bar{\rho}_{\infty}\right)+\nu \frac{\alpha+\beta-2}{2} \int_{\mathbb{R}^{N}} h(x) u_{0}^{\alpha} v_{0}^{\beta} d x,
$$

hence, by (49), (32), (35), (38), and (45),

$$
J_{\nu}\left(u_{0}, v_{0}\right)=c-\frac{1}{N}\left(\sum_{k \in \mathcal{K}} \bar{\rho}_{k}+\bar{\rho}_{0}+\bar{\rho}_{\infty}\right)<\frac{S^{\frac{N}{2}}\left(\lambda_{1}\right)+S^{\frac{N}{2}}\left(\lambda_{2}\right)}{N}-\frac{S^{\frac{N}{2}}\left(\lambda_{2}\right)}{N}=\frac{1}{N} S^{\frac{N}{2}}\left(\lambda_{1}\right) .
$$

Using the definition of $S^{\frac{N}{2}}\left(\lambda_{1}\right)$ we obtain that

$$
\int_{\mathbb{R}^{N}} u_{0}^{2^{*}} d x+\alpha \nu \int_{\mathbb{R}^{N}} h(x) u_{0}^{\alpha} v_{0}^{\beta} d x=\int_{\mathbb{R}^{N}}\left|\nabla u_{0}\right|^{2} d x-\lambda_{1} \int_{\mathbb{R}^{N}} \frac{u_{0}^{2}}{|x|^{2}} d x \geq S\left(\lambda_{1}\right)\left(\int_{\mathbb{R}^{N}} u_{0}^{2^{*}} d x\right)^{\frac{2}{2^{*}}} .
$$

Setting $a:=\int_{\mathbb{R}^{N}} u_{0}^{2^{*}} d x$ and using Hölder's inequality and (49), there results that

$$
a+C \nu a^{\frac{\alpha}{2^{*}}} \geq S\left(\lambda_{1}\right) a^{\frac{2}{2^{*}}}
$$


where the constant $C>0$ depends only on $N, \lambda_{1}, \lambda_{2}, \beta, \alpha, h$, and is independent of $u_{0}, v_{0}$, and $\nu$. Since $\alpha \geq 2$, then by Lemma 3.3 there exists $\nu_{1}=\nu_{1}(\varepsilon)>0$, depending only on the data and independent of $u_{0}$ and $v_{0}$ such that, if $\nu \leq \nu_{1}$, then

$$
a \geq(1-\varepsilon) S^{\frac{N}{2}}\left(\lambda_{1}\right) \text {. }
$$

In a similar way, setting $b=\int_{\mathbb{R}^{N}} v_{0}^{2^{*}} d x$, we find some $\nu_{2}=\nu_{2}(\varepsilon)>0$ independent of $u_{0}$ and $v_{0}$ such that, if $\nu \leq \nu_{2}$, then

$$
b \geq(1-\varepsilon) S^{\frac{N}{2}}\left(\lambda_{2}\right) .
$$

From the two above estimates, (49), and the choice of $\varepsilon$, we obtain that

$$
J_{\nu}\left(u_{0}, v_{0}\right) \geq \frac{1-\varepsilon}{N}\left(S^{\frac{N}{2}}\left(\lambda_{1}\right)+S^{\frac{N}{2}}\left(\lambda_{2}\right)\right)>\frac{1}{N} S^{\frac{N}{2}}\left(\lambda_{1}\right),
$$

thus giving rise to a contradiction with (50). Hence $v_{n} \rightarrow v_{0}$ strongly in $\mathcal{D}^{1,2}\left(\mathbb{R}^{N}\right)$ and the statement is proved also in case 2 .

Remark 3.6. If $h$ is a radial function, we can perform the above concentration compactness analysis in the space $\mathbb{D}_{r}:=\mathcal{D}_{r}^{1,2}\left(\mathbb{R}^{N}\right) \times \mathcal{D}_{r}^{1,2}\left(\mathbb{R}^{N}\right)=\{(u, v) \in \mathbb{D}: u$ and $v$ are radially symmetric $\}$. In this case, assumption (44) is no more needed, due to the Strauss Lemma which ensures compactness of the inclusion $\mathcal{D}_{r}^{1,2}\left(\mathbb{R}^{N}\right)$ into $L^{p}\left(\left\{x \in \mathbb{R}^{N}: R_{1} \leq|x| \leq R_{2}\right\}\right)$ for all $p>1$ and for all $R_{2}>R_{1}>0$; in particular no concentration at points different from 0 and $\infty$ can occur.

3.2. Existence of ground state solutions. As a direct consequence of the Palais-Smale condition proved in Lemma 3.2, the following result about existence of ground state solutions holds true. As stated in Theorem 3.4, there is no hope to find non semitrivial ground state solutions to (1) for $\alpha, \beta \geq 2$, unless $\nu$ is sufficiently large.

Theorem 3.7. Let $\mathbf{( H )}$ and (21) hold.

i) There exists $\nu_{0}>0$ such that, for all $\nu \geq \nu_{0}$, (1) admits a ground state solution $(\phi, \psi) \in \mathbb{D}$ (according to Definition 2.1) such that $\phi>0$ and $\psi>0$ in $\mathbb{R}^{N} \backslash\{0\}$.

ii) If $\alpha<2$ and $\beta<2$, then (1) admits a ground state solution $(\phi, \psi) \in \mathbb{D}$ such that $\phi>0$ and $\psi>0$ in $\mathbb{R}^{N} \backslash\{0\}$.

Proof. Letting $c^{*}(\nu):=\inf _{(u, v) \in \mathcal{N}_{\nu}} J_{\nu}(u, v)$, a direct calculation shows that

$$
0<c^{*}(\nu) \leq \frac{1}{N}\left(\min \left\{S\left(\lambda_{1}\right), S\left(\lambda_{2}\right)\right\}\right)^{\frac{N}{2}} .
$$

In view of the compactness Lemma 3.2, to prove that $c^{*}(\nu)$ is attained is enough to prove that $c^{*}(\nu)<\frac{1}{N}\left(\min \left\{S\left(\lambda_{1}\right), S\left(\lambda_{2}\right)\right\}\right)^{N / 2}$. To this aim, we fix $(u, v) \in \mathbb{D}$ such $\int_{\mathbb{R}^{N}} h(x)|u|^{\alpha}|v|^{\beta} d x>0$. Let $t_{\nu}=t_{[\nu,(u, v)]}>0$ be such that $t_{\nu}(u, v) \in \mathcal{N}_{\nu}$, see $(7)$. Notice that $\lim _{\nu \rightarrow+\infty} t_{\nu}=0$ and

$$
\lim _{\nu \rightarrow+\infty} t_{\nu}^{\alpha+\beta-2} \nu=\frac{\|(u, v)\|_{\mathbb{D}}^{2}}{(\alpha+\beta) \int_{\mathbb{R}^{N}} h(x)|u|^{\alpha}|v|^{\beta} d x} .
$$

Therefore we have that

$$
J_{\nu}\left(t_{\nu}(u, v)\right)=t_{\nu}^{2}\|(u, v)\|_{\mathbb{D}}^{2}\left(\frac{1}{2}-\frac{1}{\alpha+\beta}-o(1)\right) \quad \text { as } \nu \rightarrow+\infty .
$$

Hence $J_{\nu}\left(t_{\nu}(u, v)\right)=o(1)$ as $\nu \rightarrow \infty$ and then there exists $\nu_{0}>0$ such that, if $\nu \geq \nu_{0}$, then

$$
c^{*}(\nu) \leq J_{\nu}\left(t_{\nu}(u, v)\right)<\frac{1}{N}\left(\min \left\{S\left(\lambda_{1}\right), S\left(\lambda_{2}\right)\right\}\right)^{N / 2} .
$$


The existence of a ground state $(\phi, \psi) \in \mathcal{N}_{\nu}$ for all $\nu \geq \nu_{0}$ follows easily from Lemma 3.2. By evenness of $J_{\nu}$ and of the Nehari manifold, we can assume that $\phi \geq 0, \psi \geq 0$, and, by classical regularity results, $\phi$ and $\psi$ are smooth in $\mathbb{R}^{N} \backslash\{0\}$. Moreover, $\phi \not \equiv 0$ and $\psi \neq \equiv 0$. Indeed, if, by contradiction, $\psi \equiv 0$, then $\phi \geq 0, \phi \neq 0$, solves

$$
-\Delta \phi-\lambda_{1} \frac{\phi}{|x|^{2}}=\phi^{2^{*}-1}
$$

Hence $\phi=z_{\mu}^{\lambda_{1}}$ for some $\mu>0$ and, consequently, $c^{*}=J_{\nu}(\phi, 0)=I_{\lambda_{1}}(\phi)=\frac{1}{N} S^{\frac{N}{2}}\left(\lambda_{1}\right)$, thus giving rise to a contradiction with the fact that $c^{*}<\frac{1}{N}\left(\min \left\{S\left(\lambda_{1}\right), S\left(\lambda_{2}\right)\right\}\right)^{N / 2}$. In the same way, we can prove that $\psi \neq \equiv 0$. The maximum principle in $\mathbb{R}^{N} \backslash\{0\}$ implies that $\phi>0$ and $\psi>0$ in $\mathbb{R}^{N} \backslash\{0\}$. Statement i) is thereby proved.

If $\alpha<2$ and $\beta<2$, then by Theorem 2.2, parts iii) and iv), we have that $\left(z_{1}^{\lambda_{1}}, 0\right)$ and $\left(0, z_{1}^{\lambda_{2}}\right)$ are saddle points for $J_{\nu}$ in $\mathcal{N}_{\nu}$. More precisely, in view of (19), the assumption $\beta<2$ yields that

$$
c^{*}(\nu)=\inf _{\mathcal{N}_{\nu}} J_{\nu}<J_{\nu}\left(z_{1}^{\lambda_{1}}, 0\right)
$$

and, analogously, from $\alpha<2$ we can deduce that

$$
c^{*}(\nu)=\inf _{\mathcal{N}_{\nu}} J_{\nu}<J_{\nu}\left(0, z_{1}^{\lambda_{2}}\right)
$$

Therefore, in view of $(11), c^{*}(\nu)<\frac{1}{N}\left(\min \left\{S\left(\lambda_{1}\right), S\left(\lambda_{2}\right)\right\}\right)^{N / 2}$. Using Lemma 3.2 and arguing as above, we conclude that there exists a ground state $(\phi, \psi) \in \mathcal{N}_{\nu}$ such that $\phi>0$ and $\psi>0$ in $\mathbb{R}^{N} \backslash\{0\}$.

3.3. Existence of mountain pass solutions. For $\alpha, \beta>2$ (also for $\alpha, \beta \geq 2$ if $\nu$ is small), the semi-trivial solutions turn out to be local minimum points for the functional $J_{\nu}$ restricted to the Nehari manifold, which consequently exhibits a mountain pass geometry.

The improved Palais-Smale condition provided by Lemma 3.5 and the mountain pass geometry exhibited by the functional $J_{\nu}$ for $\alpha, \beta \geq 2$, allow us to prove the existence of mountain pass critical points for $\nu$ small. We notice that the conditions $\alpha, \beta \geq 2$ and (21) can both hold only in dimension $N=3$.

Theorem 3.8. Let $(\mathbf{H})$ and (21) hold. Assume $N=3, \alpha, \beta \geq 2$, and $\lambda_{1} \leq \lambda_{2}<1 / 4$ are such that (44) and

$$
\frac{1}{2}<\frac{\Lambda_{3}-\lambda_{2}}{\Lambda_{3}-\lambda_{1}}
$$

are satisfied. Then there exists $\nu_{1}>0$ (depending only on $N, \lambda_{1}, \lambda_{2}, \alpha, \beta$, and $h$ ) such that, if $\nu \leq \nu_{1}$, the restriction of $\bar{J}_{\nu}$ on $\overline{\mathcal{N}}_{\nu}$ has a mountain pass critical point $\left\{\left(u_{0}, v_{0}\right)\right\} \in \overline{\mathcal{N}}_{\nu}$ such that $u_{0}>0$ and $v_{0}>0$ in $\mathbb{R}^{N} \backslash\{0\}$, and, consequently, problem (1) admits a positive weak solution.

Proof. Our goal is to construct a mountain pass level for the functional on the Nehari manifold at which the Palais-Smale condition holds. Let us consider the set of paths in $\overline{\mathcal{N}}_{\nu}$ joining $\left(z_{1}^{\lambda_{1}}, 0\right)$ with $\left(0, z_{1}^{\lambda_{2}}\right)$, i.e.

$$
\Sigma_{\nu}=\left\{\begin{array}{c}
\gamma=\left(\gamma_{1}, \gamma_{2}\right):[0,1] \rightarrow \overline{\mathcal{N}}_{\nu} \text { continuous such that } \\
\gamma_{1}(0)=z_{1}^{\lambda_{1}}, \gamma_{1}(1)=0, \gamma_{2}(0)=0, \gamma_{2}(1)=z_{1}^{\lambda_{2}}
\end{array}\right\}
$$

and define the associated mountain pass level

$$
C_{M P}(\nu)=\inf _{\gamma \in \Sigma} \max _{t \in[0,1]} \bar{J}_{\nu}(\gamma(t)) .
$$


By (51) we obtain that

$$
\frac{2}{N} S^{\frac{N}{2}}\left(\lambda_{2}\right)>\frac{1}{N} S^{\frac{N}{2}}\left(\lambda_{1}\right)
$$

hence, by the monotonicity of $\lambda \mapsto S(\lambda)$, there $\varepsilon_{1}>0$ sufficiently small (depending only on $N, \lambda_{1}$, and $\lambda_{2}$ ) such that

$$
\frac{2}{N}\left(1-\varepsilon_{1}\right)\left(\frac{S\left(\lambda_{1}\right)+S\left(\lambda_{2}\right)}{2}\right)^{\frac{N}{2}}>\frac{2}{N} S^{\frac{N}{2}}\left(\lambda_{2}\right)>\frac{\left(1+\varepsilon_{1}\right)}{N} S^{\frac{N}{2}}\left(\lambda_{1}\right) \quad \text { if } \lambda_{1}<\lambda_{2}
$$

and

$$
\frac{2}{N}\left(1-\varepsilon_{1}\right)\left(\frac{S\left(\lambda_{1}\right)+S\left(\lambda_{2}\right)}{2}\right)^{\frac{N}{2}}>\frac{\left(1+\varepsilon_{1}\right)}{N} S^{\frac{N}{2}}\left(\lambda_{1}\right) \text { if } \lambda_{1}=\lambda_{2} .
$$

We now claim that there exists $\nu_{1}=\nu_{1}\left(\varepsilon_{1}\right)$ such that, for $0<\nu<\nu_{1}$

$$
\max _{t \in[0,1]} \bar{J}_{\nu}(\gamma(t)) \geq \frac{2}{N}\left(1-\varepsilon_{1}\right)\left(\frac{S\left(\lambda_{1}\right)+S\left(\lambda_{2}\right)}{2}\right)^{\frac{N}{2}}, \quad \text { for all } \gamma \in \Sigma_{\nu} .
$$

Let $\left(\gamma_{1}, \gamma_{2}\right) \in \Sigma_{\nu}$. Notice that, since $\left(\gamma_{1}(t), \gamma_{2}(t)\right) \in \overline{\mathcal{N}}_{\nu}$, then

$$
\begin{aligned}
& \int_{\mathbb{R}^{N}}\left|\nabla \gamma_{1}(t)\right|^{2} d x-\lambda_{1} \int_{\mathbb{R}^{N}} \frac{\gamma_{1}^{2}(t)}{|x|^{2}} d x+\int_{\mathbb{R}^{N}}\left|\nabla \gamma_{2}(t)\right|^{2} d x-\lambda_{2} \int_{\mathbb{R}^{N}} \frac{\gamma_{2}^{2}(t)}{|x|^{2}} d x \\
& =\int_{\mathbb{R}^{N}}\left(\gamma_{1}(t)\right)_{+}^{2^{*}} d x+\int_{\mathbb{R}^{N}}\left(\gamma_{2}(t)\right)_{+}^{2^{*}} d x+\nu(\alpha+\beta) \int_{\mathbb{R}^{N}} h(x)\left(\gamma_{1}(t)\right)_{+}^{\alpha}\left(\gamma_{2}(t)\right)_{+}^{\beta} d x
\end{aligned}
$$

and

$$
\bar{J}_{\nu}\left(\gamma_{1}(t), \gamma_{2}(t)\right)=\frac{1}{N}\left(\int_{\mathbb{R}^{N}}\left(\gamma_{1}(t)\right)_{+}^{2^{*}} d x+\int_{\mathbb{R}^{N}}\left(\gamma_{2}(t)\right)_{+}^{2_{+}^{*}} d x\right)+\nu \frac{\alpha+\beta-2}{2} \int_{\mathbb{R}^{N}} h(x)\left(\gamma_{1}(t)\right)_{+}^{\alpha}\left(\gamma_{2}(t)\right)_{+}^{\beta} d x
$$

To deduce (54), we can assume that $\max _{t \in[0,1]} \int_{\mathbb{R}^{N}}\left(\gamma_{i}(t)\right)_{+}^{2^{*}} d x \leq 2 S^{N / 2}\left(\lambda_{1}\right)$ for $i=1$, 2 without loss of generality, otherwise there is nothing to prove. Consider $f_{i}(t)=\int_{\mathbb{R}^{N}}\left(\gamma_{i}(t)\right)_{+}^{2^{*}} d x$ where $i=1,2$, then $f_{1}(0)=\int_{\mathbb{R}^{N}}\left(z_{1}^{\lambda_{1}}\right)^{2^{*}} d x>f_{2}(0)=0$ and $f_{1}(1)=0<f_{2}(1)=\int_{\mathbb{R}^{N}}\left(z_{1}^{\lambda_{2}}\right)^{2^{*}} d x$, hence, by continuity, there exists $t_{1} \in(0,1)$ such that $f_{1}\left(t_{1}\right)=f_{2}\left(t_{1}\right)>0$. From the definition of $S\left(\lambda_{1}\right)$ and $S\left(\lambda_{2}\right)$, it follows that

$$
\begin{aligned}
S\left(\lambda_{1}\right) & \left(\int_{\mathbb{R}^{N}}\left(\gamma_{1}\left(t_{1}\right)\right)_{+}^{2^{*}} d x\right)^{\frac{2}{2^{*}}}+S\left(\lambda_{2}\right)\left(\int_{\mathbb{R}^{N}}\left(\gamma_{2}\left(t_{1}\right)\right)_{+}^{2^{*}} d x\right)^{\frac{2}{2^{*}}} \\
& \leq \int_{\mathbb{R}^{N}}\left|\nabla \gamma_{1}\left(t_{1}\right)\right|^{2} d x-\lambda_{1} \int_{\mathbb{R}^{N}} \frac{\gamma_{1}^{2}\left(t_{1}\right)}{|x|^{2}} d x+\int_{\mathbb{R}^{N}}\left|\nabla \gamma_{2}\left(t_{1}\right)\right|^{2} d x-\lambda_{2} \int_{\mathbb{R}^{N}} \frac{\gamma_{2}^{2}\left(t_{1}\right)}{|x|^{2}} d x \\
& =\int_{\mathbb{R}^{N}}\left(\gamma_{1}\left(t_{1}\right)\right)_{+}^{2^{*}} d x+\int_{\mathbb{R}^{N}}\left(\gamma_{2}\left(t_{1}\right)\right)_{+}^{2^{*}} d x+\nu(\alpha+\beta) \int_{\mathbb{R}^{N}} h(x)\left(\gamma_{1}\left(t_{1}\right)\right)_{+}^{\alpha}\left(\gamma_{2}\left(t_{1}\right)\right)_{+}^{\beta} d x .
\end{aligned}
$$

We set $s=\int_{\mathbb{R}^{N}}\left(\gamma_{1}\left(t_{1}\right)\right)_{+}^{2^{*}} d x=\int_{\mathbb{R}^{N}}\left(\gamma_{2}\left(t_{1}\right)\right)_{+}^{2^{*}} d x$, then, using Hölder's inequality, we obtain that

$$
\left(S\left(\lambda_{1}\right)+S\left(\lambda_{2}\right)\right) s^{\frac{2}{2^{*}}} \leq 2 s+\nu B s^{\frac{\alpha+\beta}{2^{*}}},
$$

where $B$ is a constant depending on $h, \alpha$, and $\beta$. Since $\alpha+\beta>2$, from Lemma 3.3 we deduce that there exists $\nu_{1}=\nu_{1}\left(\varepsilon_{1}\right)$ such that $s \geq\left(1-\varepsilon_{1}\right)\left(\frac{S\left(\lambda_{1}\right)+S\left(\lambda_{2}\right)}{2}\right)^{N / 2}$ for all $0<\nu<\nu_{1}$. Hence

$$
\max _{t \in[0,1]} \bar{J}_{\nu}(\gamma(t)) \geq \frac{1}{N}\left(\int_{\mathbb{R}^{N}}\left(\gamma_{1}\left(t_{1}\right)\right)_{+}^{2^{*}} d x+\int_{\mathbb{R}^{N}}\left(\gamma_{2}\left(t_{1}\right)\right)_{+}^{2^{*}} d x\right) \geq \frac{2}{N}\left(1-\varepsilon_{1}\right)\left(\frac{S\left(\lambda_{1}\right)+S\left(\lambda_{2}\right)}{2}\right)^{\frac{N}{2}},
$$


thus proving claim (54).

From (52), (53), and (54), it follows that $C_{M P(\nu)}>\frac{\left(1+\varepsilon_{1}\right)}{N} S^{\frac{N}{2}}\left(\lambda_{1}\right)=\left(1+\varepsilon_{1}\right) \bar{J}_{\nu}\left(z_{1}^{\lambda_{1}}, 0\right)$, and hence $\bar{J}_{\nu}$ exhibits a mountain pass geometry.

Let us now set $\gamma_{1}(t)=(1-t)^{1 / 2} z_{1}^{\lambda_{1}}$ and $\gamma_{2}(t)=t^{1 / 2} z_{1}^{\lambda_{2}}, t \in[0,1]$. By the definition of the Nehari Manifold, there exists a continuous positive function $k(t)$ such that $k(t)\left(\gamma_{1}(t), \gamma_{2}(t)\right) \in \overline{\mathcal{N}}_{\nu} \cap \mathcal{N}_{\nu}$ for all $t \in[0,1]$. It is clear that $k(0)=k(1)=1$. For simplicity of notation, we set

$$
a:=\left\|z_{1}^{\lambda_{1}}\right\|_{\lambda_{1}}^{2}=\int_{\mathbb{R}^{N}}\left|z_{1}^{\lambda_{1}}\right|^{2^{*}} d x=S^{\frac{N}{2}}\left(\lambda_{1}\right) \quad \text { and } \quad b:=\left\|z_{1}^{\lambda_{2}}\right\|_{\lambda_{2}}^{2}=\int_{\mathbb{R}^{N}}\left|z_{1}^{\lambda_{2}}\right|^{2^{*}} d x=S^{\frac{N}{2}}\left(\lambda_{2}\right) .
$$

From (7), it follows that

$$
\begin{aligned}
& \left\|\left((1-t)^{\frac{1}{2}} z_{1}^{\lambda_{1}}, t^{\frac{1}{2}} z_{1}^{\lambda_{2}}\right)\right\|_{\mathbb{D}}^{2} \\
& \quad=k^{2^{*}-2}(t)\left((1-t)^{\frac{2^{*}}{2}} a+t^{\frac{2^{*}}{2}} b\right)+(\alpha+\beta) \nu k^{\alpha+\beta-2}(t)(1-t)^{\frac{\alpha}{2}} t^{\frac{\beta}{2}} \int_{\mathbb{R}^{N}} h(x)\left|z_{1}^{\lambda_{1}}\right|^{\alpha}\left|z_{1}^{\lambda_{2}}\right|^{\beta} d x .
\end{aligned}
$$

Hence

$$
k^{2^{*}-2}(t)<\frac{\left\|\left(\gamma_{1}(t), \gamma_{2}(t)\right)\right\|_{\mathbb{D}}^{2}}{\int_{\mathbb{R}^{N}}\left[\left|\gamma_{1}(t)\right|^{2^{*}}+\left|\gamma_{2}(t)\right|^{2^{*}}\right]}=\frac{(1-t) a+t b}{(1-t)^{\frac{2^{*}}{2}} a+t^{\frac{2^{*}}{2}} b} \quad \text { for all } 0<t<1 .
$$

Thus from (10) we have that

$$
\bar{J}_{\nu}\left(k(t)\left(\gamma_{1}(t), \gamma_{2}(t)\right)\right)<\frac{k^{2}(t)}{N}((1-t) a+t b), \quad \text { for all } t \in(0,1),
$$

and then

$$
\max _{t \in[0,1]} \bar{J}_{\nu}\left(k(t)\left(\gamma_{1}(t), \gamma_{2}(t)\right)\right)=\bar{J}_{\nu}\left(k\left(t_{2}\right)\left(\gamma_{1}\left(t_{2}\right), \gamma_{2}\left(t_{2}\right)\right)\right)<\frac{k^{2}\left(t_{2}\right)}{N}\left(\left(1-t_{2}\right) a+t_{2} b\right)
$$

for some $0<t_{2}<1$. We notice that the fact that $t_{2}$ falls inside $(0,1)$ is due to Remark 2.3. From (55), we obtain that

$$
k\left(t_{2}\right)<\left[\frac{\left(1-t_{2}\right) a+t_{2} b}{\left(1-t_{2}\right)^{\frac{2 *}{2}} a+t_{2}^{\frac{2 *}{2}} b}\right]^{\frac{N-2}{4}} .
$$

Therefore we conclude that

$$
\max _{t \in[0,1]} \bar{J}_{\nu}\left(k(t)\left(\gamma_{1}(t), \gamma_{2}(t)\right)\right)<\frac{1}{N}\left[\frac{\left(1-t_{2}\right) a+t_{2} b}{\left(1-t_{2}\right)^{\frac{2^{*}}{2}} a+t_{2}^{\frac{2^{*}}{2}} b}\right]^{\frac{N-2}{2}}\left(\left(1-t_{2}\right) a+t_{2} b\right) .
$$

Setting

$$
g(t):=\frac{1}{N}\left[\frac{(1-t) a+t b}{(1-t)^{\frac{2 *}{2}} a+t^{\frac{2^{*}}{2}} b}\right]^{\frac{N-2}{2}}((1-t) a+t b),
$$

a direct computation shows that $g(t)$ achieves its maximum at $t=1 / 2$ and $g(1 / 2)=\frac{1}{N}(a+b)$. Hence

$$
C_{M P}(\nu) \leq \max _{t \in[0,1]} \bar{J}_{\nu}\left(k(t)\left(\gamma_{1}(t), \gamma_{2}(t)\right)\right)<\frac{1}{N}\left(S^{\frac{N}{2}}\left(\lambda_{1}\right)+S^{\frac{N}{2}}\left(\lambda_{2}\right)\right) .
$$

Therefore, from above, (52), (53), and (54), we conclude that, for all $0<\nu<\nu_{1}$,

$$
\frac{1}{N} S^{\frac{N}{2}}\left(\lambda_{1}\right)<C_{M P}(\nu)<\frac{2}{N} S^{\frac{N}{2}}\left(\lambda_{1}\right) \quad \text { if } \lambda_{1}=\lambda_{2}
$$


and, due to (51),

$$
\frac{1}{N} S^{\frac{N}{2}}\left(\lambda_{1}\right)<\frac{2}{N} S^{\frac{N}{2}}\left(\lambda_{2}\right)<C_{M P}(\nu)<\frac{1}{N}\left(S^{\frac{N}{2}}\left(\lambda_{1}\right)+S^{\frac{N}{2}}\left(\lambda_{2}\right)\right)<\frac{3}{N} S^{\frac{N}{2}}\left(\lambda_{2}\right) \quad \text { if } \lambda_{1}<\lambda_{2} .
$$

In particular $C_{M P}(\nu)$ satisfies assumptions (45) and (46) of Lemma 3.5.

By the classical Mountain Pass Theorem, there exists a sequence $\left\{\left(u_{n}, v_{n}\right)\right\}_{n} \subset \overline{\mathcal{N}}_{\nu}$ such that

$$
\bar{J}_{\nu}\left(u_{n}, v_{n}\right) \rightarrow C_{M P}(\nu),\left.\quad\left(\bar{J}_{\nu}\right)^{\prime}\right|_{\overline{\mathcal{N}}_{\nu}}\left(u_{n}, v_{n}\right) \rightarrow 0, \text { and } \bar{J}_{\nu}\left(u_{n}, v_{n}\right)>\frac{\left(1+\varepsilon_{1}\right)}{N} S^{N / 2}\left(\lambda_{1}\right)
$$

Therefore, Lemma 3.5 and the analogous of Lemma 3.1 for $\bar{J}_{\nu}$ imply that $\left\{\left(u_{n}, v_{n}\right)\right\}_{n \in \mathbb{N}}$ admits a subsequence which converges strongly to a critical point $\left(u_{0}, v_{0}\right)$ of $\left.\bar{J}_{\nu}\right|_{\mathcal{N}_{\nu}}$ which is also a critical point of $\bar{J}_{\nu}$ in $\mathbb{D}$. Since $u_{0} \geq 0, u_{0} \neq \equiv 0$, and $v_{0} \geq 0, v_{0} \neq \equiv 0$, the strong maximum principle yields that $u_{0}>0$ and $v_{0}>0$ in $\mathbb{R}^{N} \backslash\{0\}$.

\section{Remark 3.9.}

(1) If $\lambda_{1}=\lambda_{2}$, then condition (51) is trivially satisfied.

(2) Condition (51) is satisfied if the values $\lambda_{1}$ and $\lambda_{2}$ are quite closed one to each other and is needed to ensure the existence of a submanifold in the Nehari manifold which separates the two manifolds $\left\{\left(z_{\mu}^{\lambda_{1}}, 0\right), \mu>0\right\}$ and $\left\{\left(0, z_{\mu}^{\lambda_{2}}\right), \mu>0\right\}$ of local minima and which stays at a higher energy level with respect to them.

Remark 3.10. Let us now consider the case where $\alpha<2<\beta$ and $\lambda_{1}=\lambda_{2}$. Arguing as in the proof of Theorem 2.2, we can verify that $\left(z_{1}^{\lambda_{1}}, 0\right)$ is a local minimum of $\bar{J}_{\nu}$ on $\overline{\mathcal{N}}_{\nu}$ and $\left(0, z_{1}^{\lambda_{2}}\right)$ is a saddle point lying at the same energy level $\frac{1}{N} S^{N / 2}\left(\lambda_{1}\right)=\frac{1}{N} S^{N / 2}\left(\lambda_{2}\right)$. Moreover, it is easy to verify that a counterpart of Lemma 3.2 for $\bar{J}_{\nu}$ holds. Hence the minimum of $\bar{J}_{\nu}$ on $\overline{\mathcal{N}}_{\nu}$ is achieved by some $\left(\phi_{\nu}, \psi_{\nu}\right) \in \mathbb{D}$ such that $\phi_{\nu}>0, \psi_{\nu}>0$ in $\mathbb{R}^{N} \backslash\{0\}$. Since $\phi_{\nu}$ and $\psi_{\nu}$ are positive, it is easy to verify that the minimum of $\bar{J}_{\nu}$ on $\overline{\mathcal{N}}_{\nu}$ is equal to the minimum of $J_{\nu}$ on $\mathcal{N}_{\nu}$, hence, setting $\lambda=\lambda_{1}=\lambda_{2}$,

$$
\begin{aligned}
c^{*}(\nu)=\bar{J}_{\nu}\left(\phi_{\nu}, \psi_{\nu}\right) & =\min _{\left\{(u, v) \in \overline{\mathcal{N}}_{\nu}\right\}} \bar{J}_{\nu}(u, v) \\
& =\min _{\left\{(u, v) \in \mathcal{N}_{\nu}\right\}} J_{\nu}(u, v)<\frac{S^{N / 2}(\lambda)}{N}=\bar{J}_{\nu}\left(z_{1}^{\lambda}, 0\right)=\bar{J}_{\nu}\left(0, z_{1}^{\lambda}\right) .
\end{aligned}
$$

It follows that the functional $\bar{J}_{\nu}$ has a mountain pass geometry and then an other non trivial critical point can exist at least for $\nu$ small. In this direction, the following results can be proved:

(1) adapting the computation performed in the proof of Lemma 3.5, it turns out that $\bar{J}_{\nu}$ satisfies the Palais-Smale condition at level $c \in \mathbb{R}$ with

$$
\frac{1}{N} S^{N / 2}(\lambda)<c<\frac{1}{N} S^{N / 2}(\lambda)+c^{*}(\nu)
$$

(2) setting

$$
\widetilde{\Sigma}_{\nu}:=\left\{\gamma=\left(\gamma_{1}, \gamma_{2}\right) \in C^{0}\left([0,1], \overline{\mathcal{N}}_{\nu}\right): \gamma_{1}(0)=z_{1}^{\lambda}, \gamma_{1}(1)=\phi_{\nu}, \gamma_{2}(0)=0, \gamma_{2}(1)=\psi_{\nu}\right\}
$$

and

$$
C_{M P 1}(\nu)=\inf _{\gamma \in \widetilde{\Sigma}_{\nu}} \max _{t \in[0,1]} \bar{J}_{\nu}(\gamma(t)),
$$

the separability condition holds, namely, for some $\varepsilon_{0}>0$ and for $\nu$ sufficiently small,

$$
\max _{t \in[0,1]} \bar{J}_{\nu}(\gamma(t))>\frac{\left(1+\varepsilon_{0}\right)}{N} S^{N / 2}\left(\lambda_{1}\right), \quad \text { for all } \gamma \in \widetilde{\Sigma}_{\nu}
$$


On the other hand, to prove the existence of a mountain pass solution, one should show the existence of a path $\left(\gamma_{1}(t), \gamma_{2}(t)\right) \in \widetilde{\Sigma}_{\nu}$ such that

$$
\max _{t \in[0,1]} \bar{J}_{\nu}\left(\gamma_{1}(t), \gamma_{2}(t)\right)<\frac{1}{N} S^{\frac{N}{2}}(\lambda)+c^{*}(\nu)
$$

and this seems to be an open problem.

\section{Critical case: variational approach.}

In this section we deal with a critical coupling term, i.e. we consider the case

$$
\alpha>1, \quad \beta>1, \quad \alpha+\beta=2^{*} \text {. }
$$

The further criticality produces an additional difficulty in proving the Palais-Smale condition. Let $\left\{\left(u_{n}, v_{n}\right)\right\}_{n \in \mathbb{N}}$ be a Palais-Smale sequence of $J_{\nu}$, i.e. $\left\{\left(u_{n}, v_{n}\right)\right\}_{n \in \mathbb{N}}$ satisfies (24). Arguing as in section 3 , it is easy to prove that $\left\{\left(u_{n}, v_{n}\right)\right\}_{n \in \mathbb{N}}$ is bounded in $\mathbb{D}$. Repeating the arguments used in the proof of Lemma 3.2 to prove strong convergence below the level $\frac{1}{N}\left(\min \left\{S\left(\lambda_{1}\right), S\left(\lambda_{2}\right)\right\}\right)^{N / 2}$, the only term which requires an extra care is $\int_{\mathbb{R}^{N}} h(x)\left|u_{n}\right|^{\alpha}\left|v_{n}\right|^{\beta} d x$, due to its criticality. To deal with such a term, we assume that $h$ vanishes at 0 and at $\infty$. More precisely $h$ is required to satisfy

$\left(\mathbf{H}_{1}\right) \quad\left\{\begin{array}{l}h \in L^{\infty}\left(\mathbb{R}^{N}\right), h \geq 0, h \not \equiv 0, h \text { is continuous in a neighborhood of } 0 \text { and } \infty, \\ h(0)=\lim _{|x| \rightarrow \infty} h(x)=0 .\end{array}\right.$

The above assumption is enough to exclude the possibility of concentration at 0 and at $\infty$. Since, if $h$ is radial, we can work in the space $\mathbb{D}_{r}=\mathcal{D}_{r}^{1,2}\left(\mathbb{R}^{N}\right) \times \mathcal{D}_{r}^{1,2}\left(\mathbb{R}^{N}\right)$ of pairs of radial $\mathcal{D}^{1,2}\left(\mathbb{R}^{N}\right)$ functions where concentration at 0 and at $\infty$ are the unique possible reasons for lack of compactness, we obtain that Palais-Smale condition holds true for $h$ radial satisfying $\left(\mathbf{H}_{1}\right)$ below level $\frac{1}{N}\left(\min \left\{S\left(\lambda_{1}\right), S\left(\lambda_{2}\right)\right\}\right)^{N / 2}$. On the other hand, in the case of a nonradial $h$, also concentration outside 0 and $\infty$ must be analyzed, and Palais-Smale condition can be proved under the additional assumption of smallness of $\nu$. Hence we treat the two cases separately.

4.1. Radial case. If $h$ is a radial function satisfying $\left(\mathbf{H}_{1}\right)$, then the following Palais-Smale condition holds.

Lemma 4.1. Let us assume that $h$ is radial, $\left(\mathbf{H}_{\mathbf{1}}\right)$ and (56) hold.

i) If $\left\{\left(u_{n}, v_{n}\right)\right\}_{n} \subset \mathbb{D}_{r}$ is a Palais-Smale sequence for $\left.J_{\nu}\right|_{\mathbb{D}_{r}}$ at level $c<\frac{1}{N}\left(\min \left\{S\left(\lambda_{1}\right), S\left(\lambda_{2}\right)\right\}\right)^{\frac{N}{2}}$, then $\left\{\left(u_{n}, v_{n}\right)\right\}_{n}$ admits a subsequence strongly converging in $\mathbb{D}$.

ii) If $\alpha, \beta \geq 2$, either $N=3$ or $N=4,0<\lambda_{1} \leq \lambda_{2}<(N-2)^{2} / 4$, and $\left\{\left(u_{n}, v_{n}\right)\right\}_{n} \subset \mathbb{D}_{r}$ is a Palais-Smale sequence for $\left.\bar{J}_{\nu}\right|_{\mathbb{D}_{r}}$ al level $c \in \mathbb{R}$ satisfying (45) and (46), then there exists $\nu_{1}>0$ (depending only on $N, \lambda_{1}, \lambda_{2}, \alpha, \beta$, and $h$ ) such that if $\nu \leq \nu_{1}$, then $\left\{\left(u_{n}, v_{n}\right)\right\}_{n}$ admits a subsequence strongly converging in $\mathbb{D}$.

Proof. Following the lines of the proofs of Lemmas 3.2 and 3.5, it is enough to prove that

$$
\lim _{\varepsilon \rightarrow 0} \limsup _{n \rightarrow \infty} \int_{\mathbb{R}^{N}} h(x)\left|u_{n}(x)\right|^{\alpha}\left|v_{n}(x)\right|^{\beta} \phi_{0}^{\varepsilon}(x) d x=0,
$$

where, for $\varepsilon>0, \phi_{0}^{\varepsilon}$ is a smooth cut-off function centered at $0,0 \leq \phi_{j}^{\varepsilon}(x) \leq 1$, satisfying

$$
\phi_{0}^{\varepsilon}(x)=\left\{\begin{array}{ll}
1, & \text { if }|x| \leq \varepsilon / 2, \\
0, & \text { if }|x| \geq \varepsilon,
\end{array} \quad \text { and } \quad\left|\nabla \phi_{0}^{\varepsilon}(x)\right| \leq \frac{4}{\varepsilon} \quad \text { for all } x \in \mathbb{R}^{N}\right.
$$


and

$$
\lim _{R \rightarrow \infty} \limsup _{n \rightarrow \infty} \int_{|x|>R} h(x)\left|u_{n}(x)\right|^{\alpha}\left|v_{n}(x)\right|^{\beta} \phi_{R}(x) d x=0
$$

where, for $R>0, \phi_{R}$ is a suitable cut-off function supported near $\infty$, such that $0 \leq \phi_{R}(x) \leq 1$,

$$
\phi_{R}(x)=\left\{\begin{array}{ll}
1, & \text { if }|x|>R+1, \\
0, & \text { if }|x|<R,
\end{array} \quad \text { and } \quad\left|\nabla \phi_{R}(x)\right| \leq \frac{4}{R} \quad \text { for all } x \in \mathbb{R}^{N} .\right.
$$

Let us prove (57). We have that

$$
\int_{\mathbb{R}^{N}} h\left|u_{n}\right|^{\alpha}\left|v_{n}\right|^{\beta} \phi_{0}^{\varepsilon} d x \leq\left(\int_{\mathbb{R}^{N}} h\left|u_{n}\right|^{2^{*}} \phi_{0}^{\varepsilon} d x\right)^{\frac{\alpha}{2^{*}}}\left(\int_{\mathbb{R}^{N}} h\left|v_{n}\right|^{2^{*}} \phi_{0}^{\varepsilon} d x\right)^{\frac{\beta}{2^{*}}} .
$$

Using the same notation of the proof of Lemma 3.2, see (26), there holds

$$
\lim _{n \rightarrow \infty} \int_{\mathbb{R}^{N}} h\left|u_{n}\right|^{2^{*}} \phi_{0}^{\varepsilon} d x=\int_{\mathbb{R}^{N}} h\left|u_{0}\right|^{2^{*}} \phi_{0}^{\varepsilon} d x+\rho_{0} h(0) \leq \int_{|x| \leq \varepsilon} h\left|u_{0}\right|^{2^{*}} d x
$$

and

$$
\lim _{n \rightarrow \infty} \int_{\mathbb{R}^{N}} h\left|v_{n}\right|^{2^{*}} \phi_{0}^{\varepsilon} d x=\int_{\mathbb{R}^{N}} h\left|v_{0}\right|^{2^{*}} \phi_{0}^{\varepsilon} d x+\bar{\rho}_{0} h(0) \leq \int_{|x| \leq \varepsilon} h\left|v_{0}\right|^{2^{*}} d x,
$$

hence there results that

$$
\lim _{\varepsilon \rightarrow 0} \limsup _{n \rightarrow \infty} \int_{\mathbb{R}^{N}} h(x)\left|u_{n}(x)\right|^{\alpha}\left|v_{n}(x)\right|^{\beta} \phi_{0}^{\varepsilon}(x) d x=0 .
$$

In a similar way, since $h$ vanishes at $\infty$, we can prove (58). Therefore, using (57) and (58), and working in the space of radial $\mathcal{D}^{1,2}\left(\mathbb{R}^{N}\right)$-functions (thus excluding concentration outside 0 and $\infty$ ), we can follow the proofs of Lemmas 3.2 and 3.5 and reach the conclusion.

Following closely the proofs of Theorems 3.7 and 3.8, the following existence result can be derived.

Theorem 4.2. Assume that $h$ is a radial function satisfying $\left(\mathbf{H}_{1}\right)$ and that (56) holds.

(1) If $\nu$ is large enough, then $J_{\nu}$ has a ground state $(\phi, \psi)$ such that $\phi>0$ and $\psi>0$ in $\mathbb{R}^{N} \backslash\{0\}$.

(2) If $N \geq 5, \alpha<2$, and $\beta<2$, then $\left(z_{\mu}^{\lambda_{1}}, 0\right)$, $\left(0, z_{\mu}^{\lambda_{2}}\right), \mu>0$, are saddle points of $J_{\nu}$ in $\mathcal{N}_{\nu} \cap \mathbb{D}_{r}$ and $J_{\nu}$ has a ground state $(\phi, \psi)$ such that $\phi>0$ and $\psi>0$ in $\mathbb{R}^{N} \backslash\{0\}$.

(3) If $N=3,4, \alpha \geq 2, \beta \geq 2, \lambda_{1} \leq \lambda_{2}<(N-2)^{2} / 4$, and

$$
\frac{\Lambda_{N}-\lambda_{2}}{\Lambda_{N}-\lambda_{1}}>2^{-\frac{2}{N-1}}
$$

there exists $\nu_{1}>0$ such that, if $\nu \leq \nu_{1}$, then the restriction of $\bar{J}_{\nu}$ on $\overline{\mathcal{N}}_{\nu} \cap \mathbb{D}_{r}$ has a mountain pass critical point $(\phi, \psi) \in \overline{\mathcal{N}}_{\nu} \cap \mathbb{D}_{r}$ such that $\phi>0$ and $\psi>0$ in $\mathbb{R}^{N} \backslash\{0\}$, and, consequently, problem (1) admits a positive radial weak solution.

We notice that conditions (56), $\alpha<2$, and $\beta<2$ can be all satisfied only in dimensions $N \geq 5$, whereas conditions (56), $\alpha \geq 2$, and $\beta \geq 2$ can be all verified only in dimensions $N=3$ and $N=4$. 
4.2. General case. The general case of a non radial function $h$ exhibits the additional problem of a possible concentration outside 0 and $\infty$ due to the criticality of the coupling term. This difficulty can be overcome if $\nu$ is sufficiently small.

Lemma 4.3. Let $\left(\mathbf{H}_{\mathbf{1}}\right)$ and (56) hold, and assume that $\left\{\left(u_{n}, v_{n}\right)\right\}_{n \in \mathbb{N}} \in \mathbb{D}$ is a Palais-Smale sequence for $J_{\nu}$ at level $c$, with

$$
c<\frac{1}{N}\left(\min \left\{S\left(\lambda_{1}\right), S\left(\lambda_{2}\right)\right\}\right)^{\frac{N}{2}}
$$

then there exists $\nu_{1}>0$ such that, if $\nu \leq \nu_{1},\left(u_{n}, v_{n}\right)$ converges strongly in $\mathbb{D}$ up to a subsequence.

PROOF. As in the proof of Lemma 4.1, it is clear that (57) and (58) hold and then we can exclude any concentration at 0 and at $\infty$ arguing as in Lemma 3.2. Therefore we have to analyze the concentration at points $x_{j} \neq 0, \infty$. Without loss of generality we can assume that $x_{j} \in \mathcal{J} \cap \mathcal{K}$ otherwise it is easy to verify that

$$
\underset{\varepsilon \rightarrow 0}{\limsup } \limsup _{n \rightarrow \infty} \int_{\mathbb{R}^{N}} h(x)\left|u_{n}\right|^{\alpha}\left|v_{n}\right|^{\beta} \phi_{j}^{\varepsilon} d x=0,
$$

where, for $\varepsilon>0, \phi_{j}^{\varepsilon}$ is a smooth cut-off function centered at $x_{j}, 0 \leq \phi_{j}^{\varepsilon}(x) \leq 1$, satisfying

$$
\phi_{j}^{\varepsilon}(x)=\left\{\begin{array}{ll}
1, & \text { if }\left|x-x_{j}\right| \leq \varepsilon / 2, \\
0, & \text { if }\left|x-x_{j}\right| \geq \varepsilon,
\end{array} \quad \text { and } \quad\left|\nabla \phi_{j}^{\varepsilon}(x)\right| \leq \frac{4}{\varepsilon} \quad \text { for all } x \in \mathbb{R}^{N},\right.
$$

and then no concentration can occur. Testing $J_{\nu}^{\prime}\left(u_{n}, v_{n}\right)$ with $\left(u_{n} \phi_{j}^{\varepsilon}, 0\right)$ we obtain

$$
\begin{aligned}
0 & =\lim _{n \rightarrow \infty}\left\langle J_{\nu}^{\prime}\left(u_{n}, v_{n}\right),\left(u_{n} \phi_{j}^{\varepsilon}, 0\right)\right\rangle \\
& =\lim _{n \rightarrow \infty}\left(\int_{\mathbb{R}^{N}}\left[\left|\nabla u_{n}\right|^{2} \phi_{j}^{\varepsilon}+u_{n} \nabla u_{n} \cdot \nabla \phi_{j}^{\varepsilon}-\lambda_{1} \frac{u_{n}^{2} \phi_{j}^{\varepsilon}}{|x|^{2}}-\phi_{j}^{\varepsilon}\left|u_{n}\right|^{2^{*}}-\alpha \nu h(x)\left|u_{n}\right|^{\alpha}\left|v_{n}\right|^{\beta} \phi_{j}^{\varepsilon}\right] d x\right) .
\end{aligned}
$$

In the same way, testing with $\left(0, v_{n} \phi_{j}^{\varepsilon}\right)$, there results that

$$
\begin{aligned}
0 & =\lim _{n \rightarrow \infty}\left\langle J_{\nu}^{\prime}\left(u_{n}, v_{n}\right),\left(0, v_{n} \phi_{j}^{\varepsilon}\right)\right\rangle \\
& =\lim _{n \rightarrow \infty}\left(\int_{\mathbb{R}^{N}}\left[\left|\nabla v_{n}\right|^{2} \phi_{j}^{\varepsilon}+v_{n} \nabla v_{n} \cdot \nabla \phi_{j}^{\varepsilon}-\lambda_{2} \frac{v_{n}^{2} \phi_{j}^{\varepsilon}}{|x|^{2}}-\phi_{j}^{\varepsilon}\left|v_{n}\right|^{2^{*}}-\beta \nu h(x)\left|u_{n}\right|^{\alpha}\left|v_{n}\right|^{\beta} \phi_{j}^{\varepsilon}\right] d x\right) .
\end{aligned}
$$

Since

$$
\int_{\mathbb{R}^{N}} h(x)\left|u_{n}\right|^{\alpha}\left|v_{n}\right|^{\beta} \phi_{j}^{\varepsilon} d x \leq\left(\int_{\mathbb{R}^{N}} h(x)\left|u_{n}\right|^{2^{*}} \phi_{j}^{\varepsilon} d x\right)^{\frac{\alpha}{2^{*}}}\left(\int_{\mathbb{R}^{N}} h(x)\left|v_{n}\right|^{2^{*}} \phi_{j}^{\varepsilon} d x\right)^{\frac{\beta}{2^{*}}}
$$

then using the fact that $h$ is bounded we obtain that, for some $\tilde{c}>0$,

$$
\limsup _{\varepsilon \rightarrow 0} \limsup _{n \rightarrow \infty} \int_{\Omega} h(x)\left|u_{n}\right|^{\alpha}\left|v_{n}\right|^{\beta} \phi_{j}^{\varepsilon} d x \leq \tilde{c} \rho_{j}^{\frac{\alpha}{2^{*}}} \bar{\rho}_{j}^{\frac{\beta}{2^{*}}},
$$

where we are using the same notation of the proof of Lemma 3.2 , see (26). Hence letting $\varepsilon \rightarrow 0$ we conclude that

$$
\mu_{j}-\rho_{j}-\tilde{c} \alpha \nu \rho_{j}^{\frac{\alpha}{2^{*}}} \bar{\rho}_{j}^{\frac{\beta}{2^{*}}} \leq 0 \text { and } \bar{\mu}_{j}-\bar{\rho}_{j}-\tilde{c} \beta \nu \rho_{j}^{\frac{\alpha}{2^{*}}} \bar{\rho}_{j}^{\frac{\beta}{2^{*}}} \leq 0 .
$$

Since $S \rho_{j}^{2 / 2^{*}} \leq \mu_{j}$ and $S \bar{\rho}_{j}^{2 / 2^{*}} \leq \bar{\mu}_{j}$, then by adding the above inequalities we obtain that

$$
S\left(\rho_{j}^{2 / 2^{*}}+\bar{\rho}_{j}^{2 / 2^{*}}\right) \leq \rho_{j}+\bar{\rho}_{j}+2^{*} \tilde{c} \nu \rho_{j}^{\frac{\alpha}{2^{*}}} \bar{\rho}_{j}^{\frac{\beta}{2^{*}}} .
$$


Thus

$$
S\left(\rho_{j}+\bar{\rho}_{j}\right)^{2 / 2^{*}} \leq\left(\rho_{j}+\bar{\rho}_{j}\right)\left(1+2^{*} \tilde{c} \nu\right)
$$

hence we conclude that either $\left(\rho_{j}+\bar{\rho}_{j}\right)=0$ or $\left(\rho_{j}+\bar{\rho}_{j}\right) \geq\left(\frac{S}{1+2^{*} \tilde{c} \nu}\right)^{N / 2}$. Hence as in (39), if concentration at $x_{j}$ occurs, we obtain that

$$
\begin{aligned}
c & \geq\left(\frac{1}{2}-\frac{1}{\alpha+\beta}\right)\left(\mu_{j}+\bar{\mu}_{j}\right)+\left(\frac{1}{\alpha+\beta}-\frac{1}{2^{*}}\right)\left(\rho_{j}+\bar{\rho}_{j}\right) \\
& \geq S\left(\frac{1}{2}-\frac{1}{\alpha+\beta}\right)\left(\rho_{j}+\bar{\rho}_{j}\right)^{2 / 2^{*}}+\left(\frac{1}{\alpha+\beta}-\frac{1}{2^{*}}\right)\left(\rho_{j}+\bar{\rho}_{j}\right) \\
& \geq \frac{1}{N}\left(\frac{S}{\left(1+2^{*} \tilde{c} \nu\right)}\right)^{\frac{N}{2}} .
\end{aligned}
$$

If $\nu$ is sufficiently small, then $c \geq \frac{1}{N}\left(\frac{S}{\left(1+2^{*} \tilde{c} \nu\right)}\right)^{N / 2} \geq \frac{1}{N}\left(\min \left\{S\left(\lambda_{1}\right), S\left(\lambda_{2}\right)\right\}\right)^{\frac{N}{2}}$, then we reach a contradiction with the hypothesis on $c$.

Remark 4.4. We notice that, while in the subcritical case the Palais-Smale condition is obtained independently of the value of $\nu$, see Lemma 3.2, to recover compactness in the case of a critical coupling term we need smallness of the parameter $\nu$.

As a direct application of Lemma 4.3, we obtain the following existence results.

Theorem 4.5. Let $\left(\mathbf{H}_{\mathbf{1}}\right)$ and (56) hold.

i) If $N \geq 5, \alpha<2$, and $\beta<2$, then there exists $\nu_{0}>0$ such that, for all $\nu \leq \nu_{0}$, (1) admits a ground state solution $(\phi, \psi) \in \mathbb{D}$ such that $\phi>0$ and $\psi>0$ in $\mathbb{R}^{N} \backslash\{0\}$.

ii) If $\lambda_{1}<\lambda_{2}$ and $\alpha<2$, then there exists $\nu_{1}>0$ such that, for all $\nu \leq \nu_{1}$, (1) admits a ground state solution $(\phi, \psi) \in \mathbb{D}$ such that $\phi>0$ and $\psi>0$ in $\mathbb{R}^{N} \backslash\{0\}$.

Proof. The proof is an easy consequence of Lemma 4.3. In case i), since the semi-trivial solutions $\left(z_{\mu}^{\lambda_{1}}, 0\right)$ and $\left(0, z_{\mu}^{\lambda_{2}}\right)$ are saddle points of $J_{\nu}$, the existence result follows by minimization on $\mathcal{N}_{\nu}$.

In case ii), since $J_{\nu}\left(z_{\mu}^{\lambda_{1}}, 0\right)>J_{\nu}\left(0, z_{\mu}^{\lambda_{2}}\right)$ and $\left(0, z_{\mu}^{\lambda_{2}}\right)$ is a saddle point, then the minimum of $J_{\nu}$ on the Nehari manifold is strictly below $\frac{1}{N}\left(\min \left\{S\left(\lambda_{1}\right), S\left(\lambda_{2}\right)\right\}\right)^{N / 2}$, and then we conclude by minimization and Lemma 4.3.

Remark 4.6. In the case where $\alpha+\beta=2^{*}, h \equiv 1$ and $\lambda_{1}=\lambda_{2}=\lambda$, then it is easy to construct, by a direct computation, positive solutions to (1) of the form $(\phi, c \phi), c>0$. More precisely:

i) If $\beta \geq \alpha$, $\phi$ solves the problem

$$
-\Delta \phi-\lambda \frac{\phi}{|x|^{2}}=\phi^{2^{*}-1}\left(1+\nu \alpha c^{\beta}\right), \phi \geq 0, \phi \in \mathcal{D}^{1,2}\left(\mathbb{R}^{N}\right) \backslash\{0\},
$$

and $c$ is a positive solution to the algebraic equation

$$
1=x^{2^{*}-2}+\nu \beta x^{\beta-2}-\nu \alpha x^{\beta},
$$

then $(\phi, c \phi)$ is a solution to (1) with $h \equiv 1$ and $\lambda_{1}=\lambda_{2}=\lambda$. We notice that solutions to (59) are suitable rescaling of $z_{1}^{\lambda}$ (see (5)), while the above algebraic equation admits at least a solution if $\beta \geq \alpha$. Indeed, if $\beta=\alpha$ then $x=1$ is a solution. If $\beta>\alpha$, then $\beta>2^{*} / 2$, and hence, setting $f(x)=x^{2^{*}-2}+\nu \beta x^{\beta-2}-\nu \alpha x^{\beta}$, there results that $f(1)>1$ and either $\beta>2$ or $\beta>2^{*}-2$. If $\beta>2$ then $\lim _{x \rightarrow 0^{+}} f(x)=0$, and hence there must exist some $\bar{x}$ such that $f(\bar{x})=1$. If $\beta>2^{*}-2$ then $\lim _{x \rightarrow+\infty} f(x)=-\infty$, and also in this case there exists $\bar{x}$ such that $f(\bar{x})=1$. 
ii) If $\beta<\alpha$, $\phi$ solves the problem

$$
-\Delta \phi-\lambda \frac{\phi}{|x|^{2}}=\phi^{2^{*}-1}\left(1+\nu \beta c^{-\alpha}\right), \phi \geq 0, \phi \in \mathcal{D}^{1,2}\left(\mathbb{R}^{N}\right) \backslash\{0\},
$$

and $c^{-1}$ is a positive solution to the algebraic equation

$$
1=x^{2^{*}-2}+\nu \alpha x^{\alpha-2}-\nu \beta x^{\alpha},
$$

then $(\phi, c \phi)$ is a solution to (1) with $h \equiv 1$ and $\lambda_{1}=\lambda_{2}=\lambda$. As above, it is easy to check that, if $\beta<\alpha$, then the above algebraic equation admits a positive solution.

\section{Critical case: perturbation argument}

In this section, a finite dimensional reduction, based on the Ambrosetti-Badiale perturbation method developed in $[3,4]$ (see also the monograph [8]), will be performed to construct solutions to problem (1) for $\alpha+\beta \leq 2^{*}$ and $\nu$ being a small perturbation parameter (not necessarily positive). Positive $\mathcal{D}^{1,2}\left(\mathbb{R}^{N}\right)$-solutions to (1) can be found as critical points of the functional $\bar{J}_{\nu}$ which can be written as

$$
\bar{J}_{\nu}(u, v)=\bar{J}_{0}(u, v)-\nu G(u, v), \quad(u, v) \in \mathbb{D},
$$

where

$$
\bar{J}_{0}(u, v)=\bar{I}_{\lambda_{1}}(u)+\bar{I}_{\lambda_{2}}(v)
$$

being the functionals $\bar{I}_{\lambda_{i}}, i=1,2$, defined in (42), and

$$
G: \mathbb{D} \rightarrow \mathbb{R}, \quad G(u, v):=\int_{\mathbb{R}^{N}} h(x) u_{+}^{\alpha} v_{+}^{\beta} d x .
$$

We observe that the unperturbed functional $\bar{J}_{0}$ has a two-dimensional manifold of non trivial and non semi-trivial critical points given by

$$
Z_{\lambda_{1}, \lambda_{2}}:=Z_{\lambda_{1}} \times Z_{\lambda_{2}}=\left\{\left(z_{\mu_{1}}^{\lambda_{1}}, z_{\mu_{2}}^{\lambda_{2}}\right), \mu_{1}>0, \mu_{2}>0\right\},
$$

where $z_{\mu_{i}}^{\lambda_{i}}$ are defined in (4-5). Through a variational perturbation argument, we are going to provide conditions on $h$ ensuring, for small $\nu$, the existence of critical points of the perturbed functional near the critical manifold $Z_{\lambda_{1}, \lambda_{2}}$. In such a variational approach, the nondegeneracy properties of the unperturbed manifold $Z_{\lambda_{1}, \lambda_{2}}$ play a key role. We say that $Z_{\lambda_{1}, \lambda_{2}}$ is nondegenerate if

$$
\operatorname{ker}\left(\bar{J}_{0}\right)^{\prime \prime}\left(z_{\mu_{1}}^{\lambda_{1}}, z_{\mu_{2}}^{\lambda_{2}}\right)=T_{\left(z_{\mu_{1}}^{\lambda_{1}}, z_{\mu_{2}}^{\lambda_{2}}\right)} Z_{\lambda_{1}, \lambda_{2}} \quad \text { for all }\left(z_{\mu_{1}}^{\lambda_{1}}, z_{\mu_{2}}^{\lambda_{2}}\right) \in Z_{\lambda_{1}, \lambda_{2}}
$$

where $\left(\bar{J}_{0}\right)^{\prime \prime}\left(z_{\mu_{1}}^{\lambda_{1}}, z_{\mu_{2}}^{\lambda_{2}}\right) \in \mathcal{L}\left(\mathbb{D}, \mathbb{D}^{\star}\right)$ denotes the second Fréchet derivative of $\bar{J}_{0}$ at $\left(z_{\mu_{1}}^{\lambda_{1}}, z_{\mu_{2}}^{\lambda_{2}}\right)$ and $T_{\left(z_{\mu_{1}}^{\lambda_{1}}, z_{\mu_{2}}^{\lambda_{2}}\right)} Z_{\lambda_{1}, \lambda_{2}}$ denotes the tangent space to $Z_{\lambda_{1}, \lambda_{2}}$ at point $\left(z_{\mu_{1}}^{\lambda_{1}}, z_{\mu_{2}}^{\lambda_{2}}\right)$.

Lemma 5.1. If $\lambda_{i} \in\left(0, \Lambda_{N}\right), i=1,2$, then (60) holds.

Proof. A direct computation shows that

$$
\operatorname{ker}\left(\bar{J}_{0}\right)^{\prime \prime}\left(z_{\mu_{1}}^{\lambda_{1}}, z_{\mu_{2}}^{\lambda_{2}}\right)=\operatorname{ker}\left(\bar{I}_{\lambda_{1}}\right)^{\prime \prime}\left(z_{\mu_{1}}^{\lambda_{1}}\right) \times \operatorname{ker}\left(\bar{I}_{\lambda_{2}}\right)^{\prime \prime}\left(z_{\mu_{2}}^{\lambda_{2}}\right) .
$$

From [13, Theorem 1.1], it follows that $\operatorname{ker}\left(\bar{I}_{\lambda_{1}}\right)^{\prime \prime}\left(z_{\mu_{1}}^{\lambda_{1}}\right)=T_{z_{\mu_{1}}{ }_{1}} Z_{\lambda_{1}}$ and $\operatorname{ker}\left(\bar{I}_{\lambda_{2}}\right)^{\prime \prime}\left(z_{\mu_{2}}^{\lambda_{2}}\right)=T_{z_{\mu_{2}}^{\lambda_{2}}} Z_{\lambda_{2}}$, hence

thus giving the conclusion.

$$
\operatorname{ker}\left(\bar{J}_{0}\right)^{\prime \prime}\left(z_{\mu_{1}}^{\lambda_{1}}, z_{\mu_{2}}^{\lambda_{2}}\right)=T_{z_{\mu_{1}}^{\lambda_{1}}} Z_{\lambda_{1}} \times T_{z_{\mu_{2}}^{\lambda_{2}}} Z_{\lambda_{2}}=T_{\left(z_{\mu_{1}}^{\lambda_{1}}, z_{\mu_{2}}^{\lambda_{2}}\right)} Z_{\lambda_{1}, \lambda_{2}},
$$


Throughout this section, we require $h$ to be a bounded function with compact support, i.e.

$\left(h_{1}\right)$

$$
\begin{aligned}
& h \in L^{\infty}\left(\mathbb{R}^{N}\right), \\
& \operatorname{supp} h \subset B_{R}:=\left\{x \in \mathbb{R}^{N}:|x|<R\right\} \text { for some } R>0 .
\end{aligned}
$$

Furthermore, some control on the behavior of $h$ at the singularity is required, i.e. we assume

$\left(h_{3}\right)$

$$
\begin{aligned}
& h(x)=O\left(|x|^{\sigma}\right) \text { as }|x| \rightarrow 0, \text { for some } \sigma \geq 0 \text { satisfying } \\
& \sigma>\max \left\{\beta\left(a_{\lambda_{2}}-a_{\lambda_{1}}\right)-2 \sqrt{1-\frac{4 \lambda_{1}}{(N-2)^{2}}}, \alpha\left(a_{\lambda_{1}}-a_{\lambda_{2}}\right)-2 \sqrt{1-\frac{4 \lambda_{2}}{(N-2)^{2}}}\right\} .
\end{aligned}
$$

It is worth noticing that $\left(\boldsymbol{h}_{\mathbf{3}}\right)$ is verified by any bounded function in the case $\lambda_{1}=\lambda_{2}$; hence if $\lambda_{1}=\lambda_{2}$, condition $\left(\boldsymbol{h}_{\mathbf{3}}\right)$ is actually contained in $\left(\boldsymbol{h}_{\mathbf{1}}\right)$. Let us also assume that exponents $\alpha, \beta$ satisfy (2) (in particular the critical case $\alpha+\beta=2^{*}$ is allowed). Since for some values of $\alpha$ and $\beta$ (i.e. for either $1<\alpha \leq 2$ or $1<\beta \leq 2) G$ may fail to be of class $C^{2}(\mathbb{D})$, we follow here the modified version of the Ambrosetti-Badiale method developed in [9] to overcome such a lack of regularity. To this aim, let us introduce the Banach space

$$
X:=\left\{(u, v) \in \mathbb{D}:\left.\left(u(x)|x|^{a_{\lambda_{1}}}\right)\right|_{B_{R}} \in L^{\infty}\left(B_{R}\right) \text { and }\left.\left(v(x)|x|^{a_{\lambda_{2}}}\right)\right|_{B_{R}} \in L^{\infty}\left(B_{R}\right)\right\}
$$

endowed with the norm

$$
\|(u, v)\|_{X}=\|(u, v)\|_{\mathbb{D}}+\underset{x \in B_{R}}{\operatorname{ess} \sup }|x|^{a_{\lambda_{1}}}|u(x)|+\underset{x \in B_{R}}{\operatorname{ess} \sup }|x|^{a_{\lambda_{2}}}|v(x)| .
$$

We now restrict our attention to a portion of the critical manifold $X$-closed to which the functional $\bar{J}_{\nu}$ recovers $C^{2}$-regularity. More precisely, we set

$$
\widetilde{Z}_{\lambda_{1}, \lambda_{2}}:=\left\{\left(z_{\mu_{1}}^{\lambda_{1}}, z_{\mu_{2}}^{\lambda_{2}}\right) \in Z_{\lambda_{1}, \lambda_{2}}: m_{1}<\mu_{1}<M_{1} \text { and } m_{2}<\mu_{2}<M_{2}\right\},
$$

with $m_{1}, m_{2}, M_{1}$, and $M_{2}$ to be chosen later on, and consider the $X$-neighborhood of $\widetilde{Z}_{\lambda_{1}, \lambda_{2}}$

$$
\mathcal{U}=\left\{\left(z_{\mu_{1}}^{\lambda_{1}}+w_{1}, z_{\mu_{2}}^{\lambda_{2}}+w_{2}\right):\left(z_{\mu_{1}}^{\lambda_{1}}, z_{\mu_{2}}^{\lambda_{2}}\right) \in \widetilde{Z}_{\lambda_{1}, \lambda_{2}},\left(w_{1}, w_{2}\right) \in X,\left\|\left(w_{1}, w_{2}\right)\right\|_{X}<\frac{a}{2}\right\},
$$

where

$$
a:=\inf \left\{\mu_{i}^{-\frac{N-2}{2}} z_{1}^{\lambda_{i}}\left(x / \mu_{i}\right)|x|^{a_{\lambda_{i}}}: x \in B_{R}, m_{i}<\mu_{i}<M_{i}, i=1,2\right\}>0 .
$$

Let us notice that, for all $(u, v)=\left(z_{\mu_{1}}^{\lambda_{1}}+w_{1}, z_{\mu_{2}}^{\lambda_{2}}+w_{2}\right) \in \mathcal{U}$ and $x \in B_{R}$, there holds

$$
\begin{aligned}
& u(x) \geq|x|^{-a_{\lambda_{1}}}\left[a-\left\|\left(w_{1}, w_{2}\right)\right\|_{X}\right]>\frac{a}{2}|x|^{-a_{\lambda_{1}}}>\frac{a}{2} R^{-a_{\lambda_{1}}}>0, \\
& v(x) \geq|x|^{-a_{\lambda_{2}}}\left[a-\left\|\left(w_{1}, w_{2}\right)\right\|_{X}\right]>\frac{a}{2}|x|^{-a_{\lambda_{2}}}>\frac{a}{2} R^{-a_{\lambda_{2}}}>0 .
\end{aligned}
$$

Therefore $\left.G\right|_{\mathcal{U}} \in C^{2}(\mathcal{U}, \mathbb{R})$. Let $\mathcal{A}_{\nu}: \mathcal{U} \rightarrow L^{\frac{2 N}{N+2}}\left(\mathbb{R}^{N}\right) \times L^{\frac{2 N}{N+2}}\left(\mathbb{R}^{N}\right)$ be given by

$$
\mathcal{A}_{\nu}(\varphi, \psi)=\left(\begin{array}{c}
\varphi_{+}^{2^{*}-1}+\nu \alpha h \varphi^{\alpha-1} \psi^{\beta} \\
\psi_{+}^{2^{*}-1}+\nu \beta h \psi^{\beta-1} \varphi^{\alpha}
\end{array}\right),
$$

and $\mathcal{R}: L^{\frac{2 N}{N+2}}\left(\mathbb{R}^{N}\right) \times L^{\frac{2 N}{N+2}}\left(\mathbb{R}^{N}\right) \rightarrow \mathbb{D}$ be the operator defined as follows: $\mathcal{R}\left(\phi_{1}, \phi_{2}\right)=\left(v_{1}, v_{2}\right)$ if and only if $\left(v_{1}, v_{2}\right)$ is the unique $\mathbb{D}$-weak solution to the linear problem

$$
\left\{\begin{array}{l}
-\Delta v_{1}-\frac{\lambda_{1}}{|x|^{2}} v_{1}=\phi_{1} \\
-\Delta v_{2}-\frac{\lambda_{2}}{|x|^{2}} v_{2}=\phi_{2}
\end{array}\right.
$$


Lemma 5.2. $\left(\mathcal{R} \circ \mathcal{A}_{\nu}\right)(\mathcal{U}) \subset X$.

Proof. To prove the lemma, it is enough to show that if $(\varphi, \psi) \in \mathcal{U}$ and $\left(v_{1}, v_{2}\right)$ is the $\mathbb{D}$-weak solution to

$$
\left\{\begin{array}{l}
-\Delta v_{1}-\frac{\lambda_{1}}{|x|^{2}} v_{1}=f \\
-\Delta v_{2}-\frac{\lambda_{2}}{|x|^{2}} v_{2}=g
\end{array}\right.
$$

with

$$
f=\varphi_{+}^{2^{*}-1}+\nu \alpha h \varphi^{\alpha-1} \psi^{\beta} \quad \text { and } \quad g=\psi_{+}^{2^{*}-1}+\nu \beta h \psi^{\beta-1} \varphi^{\alpha},
$$

then

$$
\left|v_{1}(x)\right| \leq \text { const }|x|^{-a_{\lambda_{1}}} \quad \text { and } \quad v_{2}(x) \leq \text { const }|x|^{-a_{\lambda_{2}}} \quad \text { a.e. in } B_{R} .
$$

Indeed, if $(\varphi, \psi) \in \mathcal{U}$, assumption $\left(\boldsymbol{h}_{\mathbf{3}}\right)$ ensures that there exist $s_{1}, s_{2}>N / 2$ such that

$$
\int_{\Omega}|x|^{-a_{\lambda_{1}}\left(2^{*}-2^{*} s_{1}+s_{1}\right)}|f(x)|^{s_{1}} d x<\infty \quad \text { and } \quad \int_{\Omega}|x|^{-a_{\lambda_{2}}\left(2^{*}-2^{*} s_{2}+s_{2}\right)}|g(x)|^{s_{2}} d x<\infty .
$$

Hence, from Theorem A.1 in the appendix, it follows that, for any $0<r<R$,

$$
|x|^{a_{\lambda_{1}}} v_{1}(x) \in L^{\infty}\left(B_{r}\right) \quad \text { and } \quad|x|^{a_{\lambda_{2}}} v_{2}(x) \in L^{\infty}\left(B_{r}\right) .
$$

On the other hand, standard elliptic estimates imply that $v_{1}, v_{2} \in L^{\infty}\left(B_{R} \backslash B_{r}\right)$, thus proving (61)

Lemma 5.3. $\mathcal{R} \circ \mathcal{A}_{\nu} \in C^{1}(\mathcal{U}, X)$ and

$$
d\left(\mathcal{R} \circ \mathcal{A}_{\nu}\right)\left(u_{1}, u_{2}\right)\left[v_{1}, v_{2}\right]=\left(\zeta_{1}, \zeta_{2}\right)
$$

where $\left(\zeta_{1}, \zeta_{2}\right)$ is the unique $\mathbb{D}$-weak solution to the linear problem

$$
\left\{\begin{array}{l}
-\Delta \zeta_{1}-\frac{\lambda_{1}}{|x|^{2}} \zeta_{1}=\left(2^{*}-1\right)\left(u_{1}\right)_{+}^{2^{*}-2} v_{1}+\nu \alpha(\alpha-1) h u_{1}^{\alpha-2} u_{2}^{\beta} v_{1}+\nu \alpha \beta h u_{1}^{\alpha-1} u_{2}^{\beta-1} v_{2} \\
-\Delta \zeta_{2}-\frac{\lambda_{2}}{|x|^{2}} \zeta_{2}=\left(2^{*}-1\right)\left(u_{2}\right)_{+}^{2^{*}-2} v_{2}+\nu \beta(\beta-1) h u_{2}^{\beta-2} u_{1}^{\alpha} v_{2}+\nu \beta \alpha h u_{2}^{\beta-1} u_{1}^{\alpha-1} v_{1}
\end{array}\right.
$$

Proof. By a direct calculation, it is possible to verify that $\mathcal{R} \circ \mathcal{A}_{\nu}$ is differentiable and that its Fréchet derivative is given by (62-63). Let us now show that $d\left(\mathcal{R} \circ \mathcal{A}_{\nu}\right): \mathcal{U} \rightarrow \mathcal{L}(X)$ is continuous. Indeed, let $\left(u_{1}, u_{2}\right) \in \mathcal{U}$ and $\left(u_{1}^{n}, u_{2}^{n}\right) \in \mathcal{U}$ such that $\left(u_{1}^{n}, u_{2}^{n}\right) \rightarrow\left(u_{1}, u_{2}\right)$ in $X$. We have that

$$
\begin{aligned}
& \left\|d\left(\mathcal{R} \circ \mathcal{A}_{\nu}\right)\left(u_{1}, u_{2}\right)-d\left(\mathcal{R} \circ \mathcal{A}_{\nu}\right)\left(u_{1}^{n}, u_{2}^{n}\right)\right\|_{\mathcal{L}(X)} \\
& \quad=\sup _{\left\|\left(v_{1}, v_{2}\right)\right\|_{X}=1}\left\|\left(d\left(\mathcal{R} \circ \mathcal{A}_{\nu}\right)\left(u_{1}, u_{2}\right)-d\left(\mathcal{R} \circ \mathcal{A}_{\nu}\right)\left(u_{1}^{n}, u_{2}^{n}\right)\right)\left[v_{1}, v_{2}\right]\right\|_{X} .
\end{aligned}
$$


Direct calculations yield

$$
\begin{aligned}
& \sup _{\left\|\left(v_{1}, v_{2}\right)\right\|_{X}=1}\left\|\left(d\left(\mathcal{R} \circ \mathcal{A}_{\nu}\right)\left(u_{1}, u_{2}\right)-d\left(\mathcal{R} \circ \mathcal{A}_{\nu}\right)\left(u_{1}^{n}, u_{2}^{n}\right)\right)\left[v_{1}, v_{2}\right]\right\|_{\mathbb{D}} \\
& \leq \mathrm{const} \|\left(2^{*}-1\right)\left[\left(u_{1}\right)_{+}^{2^{*}-2}-\left(u_{1}^{n}\right)_{+}^{2^{*}-2}\right] v_{1}+\nu \alpha(\alpha-1) h\left[u_{1}^{\alpha-2} u_{2}^{\beta}-\left(u_{1}^{n}\right)^{\alpha-2}\left(u_{2}^{n}\right)^{\beta}\right] v_{1} \\
& \quad+\nu \alpha \beta h\left[u_{1}^{\alpha-1} u_{2}^{\beta-1}-\left(u_{1}^{n}\right)^{\alpha-1}\left(u_{2}^{n}\right)^{\beta-1}\right] v_{2} \|_{L^{\frac{2 N}{N+2}}\left(\mathbb{R}^{N}\right)} \\
& \quad+\operatorname{const} \|\left(2^{*}-1\right)\left[\left(u_{2}\right)_{+}^{2^{*}-2}-\left(u_{2}^{n}\right)_{+}^{2^{*}-2}\right] v_{2} \\
& \quad+\nu \beta(\beta-1) h\left[u_{2}^{\beta-2} u_{1}^{\alpha}-\left(u_{2}^{n}\right)^{\beta-2}\left(u_{1}^{n}\right)^{\alpha}\right] v_{2} \\
& \quad+\nu \beta \alpha h\left[u_{2}^{\beta-1} u_{1}^{\alpha-1}-\left(u_{2}^{n}\right)^{\beta-1}\left(u_{1}^{n}\right)^{\alpha-1}\right] v_{1} \|_{L^{\frac{2 N}{N+2}}\left(\mathbb{R}^{N}\right)} \\
& \leq \mathrm{const}\left[\left\|\left(u_{1}\right)_{+}^{2^{*}-2}-\left(u_{1}^{n}\right)_{+}^{2^{*}-2}\right\|_{L^{N / 2}\left(\mathbb{R}^{N}\right)}+\left\|\left(u_{2}\right)_{+}^{2^{*}-2}-\left(u_{2}^{n}\right)_{+}^{2^{*}-2}\right\|_{L^{N / 2}\left(\mathbb{R}^{N}\right)}\right. \\
& \quad+\left\|h\left[u_{1}^{\alpha-1} u_{2}^{\beta-1}-\left(u_{1}^{n}\right)^{\alpha-1}\left(u_{2}^{n}\right)^{\beta-1}\right]\right\|_{L^{N / 2}\left(B_{R}\right)} \\
& \quad+\left\||x|^{-a_{\lambda_{1}}} \mid u_{1}^{\alpha-2} u_{2}^{\beta}-\left(u_{1}^{n}\right)^{\alpha-2}\left(u_{2}^{n}\right)^{\beta}\right\|_{L^{\frac{2 N}{N+2}}\left(B_{R}\right)} \\
& \left.\quad+\left\||x|^{-a_{\lambda_{2}}} \mid u_{2}^{\beta-2} u_{1}^{\alpha}-\left(u_{2}^{n}\right)^{\beta-2}\left(u_{1}^{n}\right)^{\alpha}\right\|_{L^{\frac{2 N}{N+2}}\left(B_{R}\right)}\right]
\end{aligned}
$$

for some const $=\operatorname{const}\left(N, \lambda_{1}, \lambda_{2}, \alpha, \beta, \nu,\|h\|_{L^{\infty}\left(\mathbb{R}^{N}\right)}\right)>0$. Strong convergences $u_{1}^{n} \rightarrow u_{1}$ and $u_{2}^{n} \rightarrow u_{2}$ in $L^{2^{*}}\left(\mathbb{R}^{N}\right)$ immediately yield

(66) $\left\|\left(u_{1}\right)_{+}^{2^{*}-2}-\left(u_{1}^{n}\right)_{+}^{2^{*}-2}\right\|_{L^{N / 2}\left(\mathbb{R}^{N}\right)}+\left\|\left(u_{2}\right)_{+}^{2^{*}-2}-\left(u_{2}^{n}\right)_{+}^{2^{*}-2}\right\|_{L^{N / 2}\left(\mathbb{R}^{N}\right)}$

$$
+\left\|h\left[u_{1}^{\alpha-1} u_{2}^{\beta-1}-\left(u_{1}^{n}\right)^{\alpha-1}\left(u_{2}^{n}\right)^{\beta-1}\right]\right\|_{L^{N / 2}\left(B_{R}\right)} \rightarrow 0 \quad \text { as } n \rightarrow+\infty .
$$

On the other hand, convergences $u_{1}^{n} \rightarrow u_{1}$ and $u_{2}^{n} \rightarrow u_{2}$ in $X$ imply pointwise convergences in $B_{R} \backslash\{0\}$ and

$$
\begin{aligned}
& |x|^{-a_{\lambda_{1}}}\left|u_{1}^{\alpha-2} u_{2}^{\beta}-\left(u_{1}^{n}\right)^{\alpha-2}\left(u_{2}^{n}\right)^{\beta}\right| \leq C|x|^{-a_{\lambda_{1}}(\alpha-1)}|x|^{-a_{\lambda_{2}} \beta} \quad \text { a.e. in } B_{R}, \\
& |x|^{-a_{\lambda_{2}}}\left|u_{2}^{\beta-2} u_{1}^{\alpha}-\left(u_{2}^{n}\right)^{\beta-2}\left(u_{1}^{n}\right)^{\alpha}\right| \leq C|x|^{-a_{\lambda_{1}} \alpha}|x|^{-a_{\lambda_{2}}(\beta-1)} \quad \text { a.e. in } B_{R},
\end{aligned}
$$

for some $C>0$ independent of $n$. Since

$$
\frac{2 N}{N+2}\left[a_{\lambda_{1}}(\alpha-1)+a_{\lambda_{2}} \beta\right]<N \quad \text { and } \quad \frac{2 N}{N+2}\left[a_{\lambda_{2}}(\beta-1)+a_{\lambda_{1}} \alpha\right]<N,
$$

it follows that $|x|^{-a_{\lambda_{1}}(\alpha-1)}|x|^{-a_{\lambda_{2}} \beta} \in L^{\frac{2 N}{N+2}}\left(B_{R}\right)$ and $|x|^{-a_{\lambda_{1}} \alpha}|x|^{-a_{\lambda_{2}}(\beta-1)} \in L^{\frac{2 N}{N+2}}\left(B_{R}\right)$, hence the Dominated Convergence Theorem ensures that

$$
\begin{aligned}
&\left\||x|^{-a_{\lambda_{1}}}\left|u_{1}^{\alpha-2} u_{2}^{\beta}-\left(u_{1}^{n}\right)^{\alpha-2}\left(u_{2}^{n}\right)^{\beta}\right|\right\|_{L^{\frac{2 N}{N+2}}\left(B_{R}\right)} \\
&+\left\||x|^{-a_{\lambda_{2}}}\left|u_{2}^{\beta-2} u_{1}^{\alpha}-\left(u_{2}^{n}\right)^{\beta-2}\left(u_{1}^{n}\right)^{\alpha}\right|\right\|_{L^{\frac{2 N}{N+2}}\left(B_{R}\right)} \rightarrow 0 \quad \text { as } n \rightarrow+\infty .
\end{aligned}
$$

On the other hand, Theorem A.1, assumption $\left(\boldsymbol{h}_{\mathbf{3}}\right)$, the above estimates, and the Dominated Convergence Theorem allow us to prove that

(68) $\sup _{\left\|\left(v_{1}, v_{2}\right)\right\|_{X}=1}\left\|\left(d\left(\mathcal{R} \circ \mathcal{A}_{\nu}\right)\left(u_{1}, u_{2}\right)-d\left(\mathcal{R} \circ \mathcal{A}_{\nu}\right)\left(u_{1}^{n}, u_{2}^{n}\right)\right)\left[v_{1}, v_{2}\right]\right\|_{L^{\infty}\left(B_{R},|x|^{a} \lambda_{1}\right) \times L^{\infty}\left(B_{R},|x|^{a} \lambda_{2}\right)}=o(1)$ as $n \rightarrow \infty$. From (64-68) we conclude that

thus completing the proof.

$$
d\left(\mathcal{R} \circ \mathcal{A}_{\nu}\right)\left(u_{1}^{n}, u_{2}^{n}\right) \rightarrow d\left(\mathcal{R} \circ \mathcal{A}_{\nu}\right)\left(u_{1}, u_{2}\right) \quad \text { in } \quad \mathcal{L}(X),
$$


Lemma 5.4. Suppose that $h$ satisfies $\left(\boldsymbol{h}_{\mathbf{1}}-\boldsymbol{h}_{\mathbf{3}}\right)$ and that (2) holds. Then there exists $\nu_{0}>0$ and a $C^{1}$ function

$$
w=\left(w_{1}, w_{2}\right):\left(m_{1}, M_{1}\right) \times\left(m_{2}, M_{2}\right) \times\left(-\nu_{0}, \nu_{0}\right) \rightarrow \mathcal{U}
$$

such that for all $m_{1}<\mu_{1}<M_{1}, m_{2}<\mu_{2}<M_{2}$, and $\nu \in\left(-\nu_{0}, \nu_{0}\right)$

$$
\begin{aligned}
& \left(w_{1}\left(\mu_{1}, \mu_{2}, \nu\right), w_{2}\left(\mu_{1}, \mu_{2}, \nu\right)\right) \text { is orthogonal to } T_{\left(z_{\mu_{1}}^{\lambda_{1}}, z_{\mu_{2}}^{\lambda_{2}}\right)} Z_{\lambda_{1}, \lambda_{2}}, \\
& \left(z_{\mu_{1}}^{\lambda_{1}}+w_{1}\left(\mu_{1}, \mu_{2}, \nu\right), z_{\mu_{2}}^{\lambda_{2}}+w_{2}\left(\mu_{1}, \mu_{2}, \nu\right)\right) \\
& \quad-\left(R \circ \mathcal{A}_{\nu}\right)\left(z_{\mu_{1}}^{\lambda_{1}}+w_{1}\left(\mu_{1}, \mu_{2}, \nu\right), z_{\mu_{2}}^{\lambda_{2}}+w_{2}\left(\mu_{1}, \mu_{2}, \nu\right)\right) \in T_{\left(z_{\mu_{1}}^{\lambda_{1}}, z_{\mu_{2}}^{\lambda_{2}}\right)} Z_{\lambda_{1}, \lambda_{2}}, \\
& \quad\left\|w\left(\mu_{1}, \mu_{2}, \nu\right)\right\|_{X}=O(\nu) \quad \text { as } \nu \rightarrow 0 .
\end{aligned}
$$

Proof. Let

be defined as

$$
H:\left(m_{1}, M_{1}\right) \times\left(m_{2}, M_{2}\right) \times \mathcal{U} \times\left(-\nu_{0}, \nu_{0}\right) \times \mathbb{R}^{2} \rightarrow X \times \mathbb{R}^{2}
$$

$$
\begin{aligned}
H_{1}\left(\mu_{1}, \mu_{2},\left(w_{1}, w_{2}\right), \nu, \alpha_{1}, \alpha_{2}\right)= & \left(z_{\mu_{1}}^{\lambda_{1}}+w_{1}, z_{\mu_{2}}^{\lambda_{2}}+w_{2}\right)-\mathcal{R} \circ \mathcal{A}_{\nu}\left(z_{\mu_{1}}^{\lambda_{1}}+w_{1}, z_{\mu_{2}}^{\lambda_{2}}+w_{2}\right) \\
& -\left(\alpha_{1} q_{\mu_{1}}^{\lambda_{1}}, \alpha_{2} q_{\mu_{2}}^{\lambda_{2}}\right), \\
H_{2}\left(\mu_{1}, \mu_{2},\left(w_{1}, w_{2}\right), \nu, \alpha_{1}, \alpha_{2}\right)= & \left(\left(w_{1}, q_{\mu_{1}}^{\lambda_{1}}\right)_{\lambda_{1}},\left(w_{2}, q_{\mu_{2}}^{\lambda_{2}}\right)_{\lambda_{2}}\right),
\end{aligned}
$$

where $q_{\mu}^{\lambda_{i}}:=\left\|\frac{d}{d \mu} z_{\mu}^{\lambda_{i}}\right\|_{\lambda_{i}}^{-1} \frac{d}{d \mu} z_{\mu}^{\lambda_{i}}, i=1,2$, is the generator of $T_{z_{\mu}^{\lambda_{i}}} Z_{\lambda_{i}}$. From Lemma (5.3), it follows that $H$ is of class $C^{1}$ and its derivative with respect to variables $\left(w_{1}, w_{2}, \alpha_{1}, \alpha_{2}\right)$ at $\left(\mu_{1}, \mu_{2},(0,0), 0,0,0\right)$ is given by

$$
\frac{\partial H}{\partial\left(w_{1}, w_{2}, \alpha_{1}, \alpha_{2}\right)}\left(\mu_{1}, \mu_{2},(0,0), 0,0,0\right)\left[v_{1}, v_{2}, b_{1}, b_{2}\right]=\left(\begin{array}{c}
\left(\bar{I}_{\lambda_{1}}\right)^{\prime \prime}\left(z_{\mu_{1}}^{\lambda_{1}}\right) v_{1}-b_{1} q_{\mu_{1}}^{\lambda_{1}} \\
\left(\bar{I}_{\lambda_{2}}\right)^{\prime \prime}\left(z_{\mu_{2}}^{\lambda_{2}}\right) v_{2}-b_{2} q_{\mu_{2}}^{\lambda_{2}} \\
\left(v_{1}, q_{\mu_{1}}^{\lambda_{1}}\right)_{\lambda_{1}} \\
\left(v_{2}, q_{\mu_{2}}^{\lambda_{2}}\right)_{\lambda_{2}}
\end{array}\right) .
$$

We notice that $\frac{\partial H}{\partial\left(w_{1}, w_{2}, \alpha_{1}, \alpha_{2}\right)}\left(\mu_{1}, \mu_{2},(0,0), 0,0,0\right) \in \mathcal{L}\left(X \times \mathbb{R}^{2}\right)$ is a Fredholm operator of index 0 ; moreover it is injective due to the nondegeneracy property provided by Lemma 5.1, hence it is invertible. The conclusion of the proof now relies on the Implicit Function Theorem and is quite standard, see e.g. [8].

Let us introduce the perturbed manifold

$$
Z_{\lambda_{1}, \lambda_{2}}^{\nu}=\left\{\left(z_{\mu_{1}}^{\lambda_{1}}+w_{1}\left(\mu_{1}, \mu_{2}, \nu\right), z_{\mu_{2}}^{\lambda_{2}}+w_{2}\left(\mu_{1}, \mu_{2}, \nu\right)\right), m_{1}<\mu_{1}<M_{1}, m_{2}<\mu_{2}<M_{2}\right\}
$$

and let $\left(z_{\nu, 1}, z_{\nu, 2}\right)$ be a critical point of $\bar{J}_{\nu}$ constrained on $Z_{\lambda_{1}, \lambda_{2}}^{\nu}$, i.e.

$$
\left(\bar{J}_{\nu}\right)^{\prime}\left(z_{\nu, 1}, z_{\nu, 2}\right)\left[v_{1}, v_{2}\right]=0 \quad \text { for all }\left(v_{1}, v_{2}\right) \in T_{\left(z_{\nu, 1}, z_{\nu, 2}\right)} Z_{\lambda_{1}, \lambda_{2}}^{\nu} .
$$

On the other hand, from (70), we deduce that

$$
\left(\overline{J_{\nu}}\right)^{\prime}\left(z_{\nu, 1}, z_{\nu, 2}\right)\left[v_{1}, v_{2}\right]=0 \quad \text { for all }\left(v_{1}, v_{2}\right) \in\left(T_{\left(z_{\mu_{1}}^{\lambda_{1}}, z_{\mu_{2}}^{\lambda_{2}}\right)} \widetilde{Z}_{\lambda_{1}, \lambda_{2}}\right)^{\perp} \text {. }
$$

Therefore, as in [9], we can conclude that critical points of $\bar{J}_{\nu}$ constrained on $Z_{\lambda_{1}, \lambda_{2}}^{\nu}$ give rise to solutions. Moreover

(72) $\bar{J}_{\nu}\left(z_{\mu_{1}}^{\lambda_{1}}+w_{1}\left(\mu_{1}, \mu_{2}, \nu\right), z_{\mu_{2}}^{\lambda_{2}}+w_{2}\left(\mu_{1}, \mu_{2}, \nu\right)\right)=\bar{J}_{0}\left(z_{\mu_{1}}^{\lambda_{1}}, z_{\mu_{2}}^{\lambda_{2}}\right)-\nu G\left(z_{\mu_{1}}^{\lambda_{1}}, z_{\mu_{2}}^{\lambda_{2}}\right)+o(\nu) \quad$ as $\nu \rightarrow 0$.

Consequently, if $\left(z_{\mu_{1}}^{\lambda_{1}}, z_{\mu_{2}}^{\lambda_{2}}\right)$ is a critical point of the restriction $\left.G\right|_{\widetilde{Z}_{\lambda_{1}, \lambda_{2}}}$ which is "stable" under small perturbations (e.g. a proper local minimum/maximum point or a non-degenerate critical 
point), then $\left(z_{\mu_{1}}^{\lambda_{1}}+w_{1}\left(\mu_{1}, \mu_{2}, \nu\right), z_{\mu_{2}}^{\lambda_{2}}+w_{2}\left(\mu_{1}, \mu_{2}, \nu\right)\right)$ turns out to be a critical point of $\bar{J}_{\nu}$, and hence a solution to system (1). In view of the above analysis, the search for solutions to system (1) is reduced to the search for "stable" critical points of the following function of two variables

$$
\Gamma\left(\mu_{1}, \mu_{2}\right):=G\left(z_{\mu_{1}}^{\lambda_{1}}, z_{\mu_{2}}^{\lambda_{2}}\right)=\int_{\mathbb{R}^{N}} h(x)\left|z_{\mu_{1}}^{\lambda_{1}}(x)\right|^{\alpha}\left|z_{\mu_{2}}^{\lambda_{2}}(x)\right|^{\beta} d x
$$

on the rectangle $\left(m_{1}, M_{1}\right) \times\left(m_{2}, M_{2}\right)$. In order to find critical points of $\Gamma$ we will assume

$\left(\boldsymbol{h}_{\mathbf{4}}\right) \quad h \neq 0$ and $h$ has a fixed $\operatorname{sign}\left(\right.$ i.e. either $h \geq 0$ or $h \leq 0$ a.e. in $\mathbb{R}^{N}$ ),

$\left(\boldsymbol{h}_{\mathbf{5}}\right) \quad \lim _{|x| \rightarrow 0} h(x) \quad$ exists and is equal to 0 .

Theorem 5.5. If $h$ satisfies $\left(\boldsymbol{h}_{\mathbf{1}}-\boldsymbol{h}_{\mathbf{5}}\right)$, then there exist $m_{1}, m_{2}, M_{1}, M_{2}$ such that $\Gamma$ admits either a proper local maximum point or a proper local minimum point in $\left(m_{1}, M_{1}\right) \times\left(m_{2}, M_{2}\right)$.

ProOF. Let us assume that $h \geq 0$ and prove that $\Gamma$ has a proper local maximum point in $\left(m_{1}, M_{1}\right) \times\left(m_{2}, M_{2}\right)$ provided $m_{1}, m_{2}$ are sufficiently small and $M_{1}, M_{2}$ are sufficiently large. Indeed, from $\left(\boldsymbol{h}_{\mathbf{4}}\right)$, it follows that

$$
\Gamma(1,1)=\int_{\mathbb{R}^{N}} h(x)\left|z_{1}^{\lambda_{1}}(x)\right|^{\alpha}\left|z_{1}^{\lambda_{2}}(x)\right|^{\beta} d x>0 .
$$

On the other hand, from Hölder's inequality we deduce that

$$
\begin{aligned}
& \Gamma\left(m_{1}, \mu_{2}\right) \leq \operatorname{const}(h)\left\|z_{1}^{\lambda_{2}}\right\|_{L^{2^{*}}\left(\mathbb{R}^{N}\right)}^{\beta}\left(\int_{\mathbb{R}^{N}}\left|h(x) \| z_{m_{1}}^{\lambda_{1}}(x)\right|^{2^{*}} d x\right)^{\frac{\alpha}{2^{*}}} \quad \text { for all } \mu_{2}>0, \\
& \Gamma\left(M_{1}, \mu_{2}\right) \leq \operatorname{const}(h)\left\|z_{1}^{\lambda_{2}}\right\|_{L^{2^{*}}\left(\mathbb{R}^{N}\right)}^{\beta}\left(\int_{\mathbb{R}^{N}}\left|h(x) \| z_{M_{1}}^{\lambda_{1}}(x)\right|^{2^{*}} d x\right)^{\frac{\alpha}{2^{*}}} \quad \text { for all } \mu_{2}>0, \\
& \Gamma\left(\mu_{1}, m_{2}\right) \leq \operatorname{const}(h)\left\|z_{1}^{\lambda_{1}}\right\|_{L^{2^{*}}\left(\mathbb{R}^{N}\right)}^{\alpha}\left(\int_{\mathbb{R}^{N}}\left|h(x) \| z_{m_{2}}^{\lambda_{2}}(x)\right|^{2^{*}} d x\right)^{\frac{\beta}{2^{*}}} \text { for all } \mu_{1}>0, \\
& \Gamma\left(\mu_{1}, M_{2}\right) \leq \operatorname{const}(h)\left\|z_{1}^{\lambda_{1}}\right\|_{L^{2^{*}}\left(\mathbb{R}^{N}\right)}^{\alpha}\left(\int_{\mathbb{R}^{N}}\left|h(x) \| z_{M_{2}}^{\lambda_{2}}(x)\right|^{2^{*}} d x\right)^{\frac{\beta}{2^{*}}} \quad \text { for all } \mu_{1}>0,
\end{aligned}
$$

for some positive constant const $(h)$ depending on $h$. From $\left(\boldsymbol{h}_{\mathbf{2}}\right),\left(\boldsymbol{h}_{\mathbf{5}}\right)$, and the Dominated Convergence Theorem, we have that

$$
\begin{aligned}
& \int_{\mathbb{R}^{N}}\left|h(x) \| z_{m_{i}}^{\lambda_{i}}(x)\right|^{2^{*}} d x=\int_{\mathbb{R}^{N}}\left|h\left(m_{i} y\right)\right|\left|z_{1}^{\lambda_{i}}(y)\right|^{2^{*}} d y \rightarrow 0 \quad \text { as } m_{i} \rightarrow 0, \quad i=1,2, \\
& \int_{\mathbb{R}^{N}}\left|h ( x ) \left\|\left.z_{M_{i}}^{\lambda_{i}}(x)\right|^{2^{*}} d x=\int_{\mathbb{R}^{N}}\left|h\left(M_{i} y\right) \| z_{1}^{\lambda_{i}}(y)\right|^{2^{*}} d y \rightarrow 0 \quad \text { as } M_{i} \rightarrow+\infty, \quad i=1,2,\right.\right.
\end{aligned}
$$

hence it is possible to choose $m_{1}, m_{2}$ sufficiently small and $M_{1}, M_{2}$ sufficiently large in order to have that

$$
\Gamma(1,1)>\max _{\partial Q} \Gamma
$$

where $Q$ is the rectangle $Q=\left(m_{1}, M_{1}\right) \times\left(m_{2}, M_{2}\right)$. Therefore $\Gamma$ must have a proper local maximum point inside the rectangle $Q$. In the same way, we can prove that, if $h \leq 0, m_{1}, m_{2}$ are sufficiently small, and $M_{1}, M_{2}$ are sufficiently large, then $\Gamma$ admits a proper local minimum point in $Q$.

As a consequence of the above discussion, we can now present the main existence result of this section. 
Theorem 5.6. Assume that $\lambda_{i} \in\left(0, \Lambda_{N}\right), i=1,2, \alpha, \beta$ satisfy (2), and $h$ verifies $\left(\boldsymbol{h}_{\mathbf{1}}-\boldsymbol{h}_{\mathbf{5}}\right)$. Then there exists $\nu_{0}>0$ such that problem (1) admits a positive solution provided $|\nu| \leq \nu_{0}$.

Proof. From Theorem 5.5, we deduce that $\Gamma$ admits either a proper local maximum point or a proper local minimum point in $\left(m_{1}, M_{1}\right) \times\left(m_{2}, M_{2}\right)$, which, in view of expansion (72), gives rise, for $\nu$ sufficiently small, to a critical points of $\bar{J}_{\nu}$ constrained on $Z_{\lambda_{1}, \lambda_{2}}^{\nu}$. Since, as remarked above, $Z_{\lambda_{1}, \lambda_{2}}^{\nu}$ is a natural constraint for $\bar{J}_{\nu}$, we obtain a critical point of $\bar{J}_{\nu}$ in $\mathbb{D}$ and hence a positive $\mathcal{D}^{1,2}\left(\mathbb{R}^{N}\right)$-solution to problem (1).

Remark 5.7. In dimension $N=3$, the case $\alpha, \beta>2$ can occur. In this case, $G$ is of class $C^{2}(\mathbb{D})$ and we can perform the finite dimensional reduction directly in $\mathbb{D}$ with no need to introduce the space $X$. Hence, when $N=3$ and $\alpha, \beta>2$, we can prove Theorem 5.6 under weaker assumption on $h$, e.g. for any $h \in L^{1}\left(\mathbb{R}^{N}\right) \cap L^{\infty}\left(\mathbb{R}^{N}\right)$ with a fixed sign and such that $h \not \equiv 0$ and $h(0)=h(\infty)=0$.

\section{Appendix A}

The following elliptic estimate for problems with Hardy-type singularities is an easy consequence of $[14$, Theorem 1.1].

Theorem A.1. Suppose $\Omega \subset \mathbb{R}^{N}$ is a bounded domain, $\lambda<(N-2)^{2} / 4$, and $u \in H^{1}(\Omega)$ weakly solves

Assume that

$$
-\Delta u-\frac{\lambda}{|x|^{2}} u=f
$$

$$
\int_{\Omega}|x|^{-a_{\lambda}\left(2^{*}-2^{*} s+s\right)}|f(x)|^{s} d x<\infty
$$

for some $s>N / 2$. Then for any $\Omega^{\prime} \Subset \Omega$ there is a constant $C=C\left(N, \Omega\right.$, dist $\left.\left(\Omega^{\prime}, \Omega\right), s\right)$ such that

$$
\left.\sup _{\Omega^{\prime}}|| x\right|^{a_{\lambda}} u(x) \mid \leq C\left\{\|u\|_{L^{2}(\Omega)}+\left(\int_{\Omega}|x|^{-a_{\lambda}\left(2^{*}-2^{*} s+s\right)}|f(x)|^{s} d x\right)^{1 / s}\right\} .
$$

Proof. It follows from [14, Theorem 1.1] after making the change of variable $v(x)=|x|^{a_{\lambda}} u(x)$.

\section{REFERENCES}

[1] B. Abdellaoui, V. Felli, I. Peral, Existence and multiplicity for perturbations of an equation involving a Hardy inequality and the critical Sobolev exponent in the whole of $\mathbb{R}^{N}$, Adv. Differential Equations, 9 (2004), no. 5-6, 481-508.

[2] N. Akhmediev, A. Ankiewicz, Partially coherent solitons on a finite background, Phys. Rev. Lett., 82 (1999), 2661-2664.

[3] A. Ambrosetti, M. Badiale, Variational perturbative methods and bifurcation of bound states from the essential spectrum, Proc. Roy. Soc. Edinburgh Sect. A, 128 (1998), no. 6, 1131-1161.

[4] A. Ambrosetti, M. Badiale, Homoclinics: Poincaré-Melnikov type results via a variational approach, Ann. Inst. H. Poincaré Anal. Non Linéaire, 15 (1998), no. 2, 233-252.

[5] A. Ambrosetti, P. H. Rabinowitz, Dual variational methods in critical point theory and applications, J. Functional Analysis, 14 (1973), 349-381.

[6] A. Ambrosetti, E. Colorado, Bound and ground states of coupled nonlinear Schrödinger equations, C. R. Math. Acad. Sci. Paris, 342 (2006), no. 7, 453-458.

[7] A. Ambrosetti, E. Colorado, and D. Ruiz, Multi-bump solitons to linearly coupled systems of nonlinear Schrödinger equations, Calc. Var. Partial Differential Equations, to appear.

[8] A. Ambrosetti, A. Malchiodi, Perturbation methods and semilinear elliptic problems on $\mathbb{R}^{n}$, Progress in Mathematics, 240, Birkhäuser Verlag, Basel, 2006. 
[9] A. Ambrosetti, J. Garcia Azorero, I. Peral, Elliptic variational problems in $\mathbb{R}^{N}$ with critical growth, Special issue in celebration of Jack K. Hale's 70th birthday, Part 1 (Atlanta, GA/Lisbon, 1998), J. Differential Equations, 168 (2000), no. 1, 10-32.

[10] M. F. Bidaut-Veron, P. Grillot, Singularities in elliptic systems with absorption terms, Ann. Scuola Norm. Sup. Pisa Cl. Sci. (4), 28 (1999), no. 4, 229-271.

[11] D.G. De Figueiredo, I. Peral, J. Rossi, The critical hyperbola for a hamiltonian elliptic system with weights, preprint.

[12] V. Felli, A. Pistoia, Existence of blowing-up solutions for a nonlinear elliptic equation with Hardy potential and critical growth, Comm. Partial Differential Equations, 31 (2006), no. 1-3, 21-56.

[13] V. Felli, M. Schneider, Perturbation results of critical elliptic equations of Caffarelli-Kohn-Nirenberg type, J. Diff. Equations, 191 (2003), 121-142.

[14] V. Felli, M. Schneider, A note on regularity of solutions to degenerate elliptic equations of Caffarelli-KohnNirenberg type, Advanced Nonlinear Studies, 3 (2003), 431-443.

[15] V. Felli, S. Terracini, Fountain-like solutions for nonlinear elliptic equations with critical growth and Hardy potential, Commun. Contemp. Math., 7 (2005), no. 6, 867-904.

[16] I.P. Kaminow, Polarization in optical fibers, IEEE J. Quantum Electron., 17 (1981), 15-22.

[17] P.L. Lions, The concentration-compactness principle in the calculus of variations. The limit case, part 1, Rev. Matemática Iberoamericana, 1 (1985), no. 1, 145-201.

[18] P.L. Lions, The concentration-compactness principle in the calculus of variations. The limit case, part 2, Rev. Matemática Iberoamericana, 1 (1985), no. 2, 45-121.

[19] T-C. Lin, J. Wei, Ground state of $N$ coupled nonlinear Schrödinger equations in $\mathbb{R}^{n}, n \leq 3$, Comm. Math. Phys., 255 (2005), no. 3, 629-653.

[20] L. A. Maia, E. Montefusco, B. Pellacci, Positive solutions for a weakly coupled nonlinear Schrödinger system, J. Differential Equations, 229 (2006), no. 2, 743-767.

[21] C.R. Menyuk, Nonlinear pulse propagation in birefringence optical fiber, IEEE J. Quantum Electron., 23, (1987), 174-176.

[22] C.R. Menyuk, Pulse propagation in an elliptically birefringent Kerr medium, IEEE J. Quantum Electron., 25 (1989), 2674-2682.

[23] E. Montefusco, B. Pellacci, M. Squassina, Semiclassical states for weakly coupled nonlinear Schrödinger system, to appear in J.E.M.S.

[24] A. Pomponio, Coupled nonlinear Schrödinger systems with potentials, J. Differential Equations, 227 (2006), no. $1,258-281$.

[25] B. Sirakov, Least Energy Solitary Waves for a System of Nonlinear Schrödinger Equations in $\mathbb{R}^{N}$, Communications in Mathematical Physics, 271 (2007), no. 1, 199-221.

[26] D. Smets, Nonlinear Schrödinger equations with Hardy potential and critical nonlinearities, Trans. AMS, 357 (2005), 2909-2938.

[27] M. Struwe, A global compactness result for elliptic boundary value problems involving limiting nonlinearities, Math.Z., 187 (1984), 511-517.

[28] S. Terracini, On positive entire solutions to a class of equations with singular coefficient and critical exponent, Adv. Diff. Equa., 1 (1996), no. 2, 241-264.

Boumediene Abdellaoui: Département de Mathématiques, Université Aboubekr Belkaïd, Tlemcen, Tlemcen 13000, Algeria. E-mail address: boumediene.abdellaoui@uam.es.

Veronica Felli: Università di Milano Bicocca, Dipartimento di Statistica, Via Bicocca degli ArcimBOLdi 8, 20126 Milano, ItAly. E-mail address: veronica.felli@unimib.it.

Ireneo Peral: Departamento de Matemáticas, Universidad Autónoma de Madrid, Cantoblanco 28049 , MADRID, SpAIN. E-mail address: ireneo.peral@uam.es. 